\title{
Planejamento periódico de trajetórias de sistemas afins sem arrasto em grupos de Lie compactos
}

\author{
Gabriel Cueva Candido Soares de Araújo
}

\author{
DissertaÇÃo APRESENTADA \\ $\mathrm{AO}$ \\ Instituto DE MATEMÁticA E Estatística \\ DA \\ Universidade De SÃo Paulo \\ PARA \\ OBTENÇÃO DO TÍTULO \\ $\mathrm{DE}$ \\ Mestre em CiênCIAS \\ Programa: Matemática Aplicada \\ Orientador: Prof. Dr. Pedro Aladar Tonelli
}

Durante o desenvolvimento deste trabalho o autor recebeu auxílio financeiro do CNPq

São Paulo, abril de 2012 


\section{Planejamento periódico de trajetórias de sistemas afins sem arrasto em grupos de Lie compactos}

Esta versão da dissertação contém as correções e alterações sugeridas pela Comissão Julgadora durante a defesa da versão original do trabalho, realizada em 08/03/2012. Uma cópia da versão original está disponível no Instituto de Matemática e Estatística da Universidade de São Paulo.

Comissão Julgadora:

- Prof. Dr. Pedro Aladar Tonelli (Presidente) - IME-USP

- Prof. Dr. Antonio Carlos Asperti - IME-USP

- Prof. Dr. Hector Bessa Silveira - UFSC 


\section{Agradecimentos}

À minha família pela ajuda, incentivo e compreensão constantes. Em particular, agradeço aos meus pais, aos meus avós, à Cláudia e à Stela por manterem um ambiente sempre receptivo e proverem, não raro com sacrifício, os meios para que eu pudesse me dedicar integralmente a este trabalho. E especialmente à Ana, por iluminar minha vida todos os dias.

Ao Pedro Tonelli, meu orientador, pela constante imposição de novos desafios e pela confiança depositada em mim durante a confecção deste trabalho, e ao Hector Bessa Silveira, membro da Comissão Julgadora, por ter lido a versão original desta dissertação em detalhe, pela intensa comunicação pessoal durante a formulação desta versão corrigida e pelas valiosas sugestões que ajudaram a melhorar muito a qualidade deste texto.

Aos professores do IMEUSP que tive o prazer de conhecer e que me ensinaram - e continuam ensinando - muito. Foi graças à inspiração e à paixão deles que escolhi estudar Matemática apesar de todas as dificuldades. Em particular agradeço à Roseli Fernandez, que me ensinou a escrever Matemática, e à Helena Maria Ávila de Castro, com quem sempre pude contar nas dificuldades da pós-graduação.

Aos amigos e colegas do IMEUSP, particularmente àqueles que frequentaram os seminários do ZFC Fã Clube e tornaram o ano de 2011 extremamente estimulante e, dentre estes, ao Luis Fernando Ragognette e ao Max Reinhold Jahnke que, mesmo sem saberem do quê se tratava, formularam um contraexemplo que me ajudou a desistir da Decomposição em Espaços de Raízes e terminar esta dissertação.

Agradeço ainda ao CNPq pelo auxílio financeiro no decorrer da confecção desta dissertação. 


\section{Resumo}

\section{ARAÚJO, G. C. C. S. Planejamento periódico de trajetórias de sistemas afins sem}

arrasto em grupos de Lie compactos. 2012. Dissertação (Mestrado) - Instituto de Matemática e Estatística, Universidade de São Paulo, São Paulo, 2012.

Tratamos o problema do planejamento periódico de trajetórias: fixados uma trajetória periódica de um sistema afim sem arrasto em um grupo de Lie compacto e conexo $G$ e uma condição inicial em $G$, encontrar outra trajetória do mesmo sistema satisfazendo a condição inicial dada e que rastreie assintoticamente a trajetória periódica dada. Resolvemos esse problema localmente (para condições iniciais em uma vizinhança de um ponto da trajetória periódica) quando $G$ é semisimples e o sistema afim é Lie-determinado (i.e. controlável), e apenas para um classe de trajetórias

periódicas (as quais denominamos regulares). Apresentamos por fim um conjunto de condições suficientes para a existência de tais trajetórias.

Palavras-chave: rastreamento de trajetórias, sistemas afins sem arrasto, grupos de Lie compactos. 


\section{Abstract}

ARAÚJO, G. C. C. S. Periodic motion planning of trajectories for control-affine driftless systems in compact Lie groups. 2012. Dissertação (Mestrado) - Instituto de Matemática e Estatística, Universidade de São Paulo, São Paulo, 2012.

We treat the periodic motion planning problem: given a periodic trajectory of a control-affine driftless system in a compact and connected Lie group $G$ and an initial condition in $G$, find another trajectory of the same system satisfying the initial condition given and that asymptotically tracks the periodic trajectory. We solve this problem locally (for initial conditions in a neighborhood of some point of the periodic trajectory) when $G$ is semisimple and the system is Lie-determined (i.e. controllable), and only for a class of periodic trajectories (which we call regular). Finally we present a set of sufficient conditions to ensure the existence of such trajectories.

Keywords: trajectory tracking, control-affine driftless systems, compact Lie groups. 


\section{Prefácio}

O objetivo principal deste trabalho é discutir o problema do planejamento periódico de trajetórias (Definição 3.2) para sistemas afins sem arrasto no contexto de grupos de Lie compactos. Para a resolução deste problema procuramos seguir, na medida do possível, a estratégia apresentada em [SdSR09] e [Sil09], Capítulo 4, onde esse problema é tratado no caso particular do grupo de Lie $\mathrm{SU}(n)$. Um dos objetivos do presente trabalho é, inclusive, generalizar as técnicas desenvolvidas nessas duas referências para nosso contexto mais abstrato. Tratamos principalmente do caso Lie-determinado (às vezes chamado na literatura de controlável).

Nos Capítulos 1 e 2 introduzimos os conceitos preliminares necessários para compreender o problema principal, introduzindo as notações e recordando os resultados básicos da teoria de variedades suaves e da teoria de Lie, assim como uma apresentação elementar da análise de famílias de campos vetoriais.

No Capítulo 3 iniciamos o assunto principal deste trabalho, enunciando o problema do planejamento periódico de trajetórias e provando que em grupos de Lie podemos reduzi-lo a um problema de estabilização de outro sistema e, fixando uma regra de escolha dos controles, à estabilidade do chamado campo auxiliar (3.9), cujo estudo passa a ser nosso objetivo.

No Capítulo 4 apresentamos alguns resultados ligados à estabilidade de campos vetoriais dependentes do tempo em variedades compactas, os quais aplicamos na sequência ao estudo dos conjuntos $\Omega$-limite do campo auxiliar provando, em linhas gerais, que este coincide com conjunto dos pontos de equilíbrio do campo auxiliar.

No Capítulo 5 introduzimos a noção de trajetória regular (Definição 5.1), a qual desfruta da seguinte propriedade: se a trajetória periódica fixada no problema do planejamento periódico for regular então o conjunto $\Omega$-limite do seu campo auxiliar associado coincide com o conjunto dos pontos críticos do caractere da representação adjunta do grupo de Lie ambiente. No caso em que o grupo de Lie em questão é semi-simples provamos que os pontos críticos que pertencem ao centro do grupo são não-degenerados e, portanto, isolados, o que com mais algum esforço resolve a questão da sua estabilidade.

Por fim, exibimos no Apêndice A um conjunto de condições suficientes para a existência de nossas trajetórias regulares, construindo uma explicitamente.

Em cada capítulo e no apêndice incorporamos uma seção "Comentários e referências", na qual discutimos com mais detalhes a bibliografia dos tópicos abordados, as hipóteses e demonstrações de alguns resultados e eventuais temas para estudo futuro, assim como restabelecer a conexão estre as técnicas desenvolvidas e a solução do problema original. 


\section{Sumário}

Prefácio $\quad$ vii

1 Famílias de campos vetoriais $\quad 1$

1.1 Notações da teoria de variedades suaves . . . . . . . . . . . . . . . . . . 1

1.2 O Teorema da Órbita . . . . . . . . . . . . . . . . . . . . 3

1.3 Famílias Lie-determinadas . . . . . . . . . . . . . . . . . . . 5

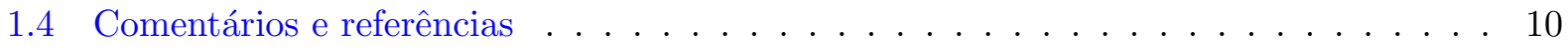

2 Tópicos da Teoria de Lie $\quad 11$

2.1 Generalidades sobre álgebras de Lie . . . . . . . . . . . . . . . . . . 11

2.2 Generalidades sobre grupos de Lie . . . . . . . . . . . . . . . . . . . . . . . . . . . . . . . . . .

2.3 O Teorema da Órbita em grupos de Lie . . . . . . . . . . . . . . . . . . 17

2.4 Particularidades dos grupos de Lie compactos . . . . . . . . . . . . . . . 21

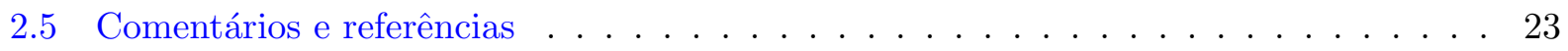

3 Planejamento periódico de trajetórias $\quad 25$

3.1 Enunciado do problema . . . . . . . . . . . . . . . . 25

3.2 Um sistema modificado . . . . . . . . . . . . . . . . . . 26

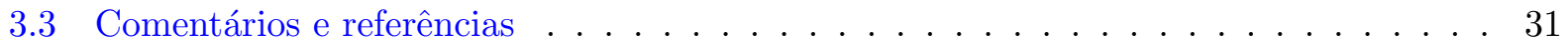

4 Estabilidade $\quad 35$

4.1 Alguns resultados de estabilidade . . . . . . . . . . . . . . . . 35

4.2 Estudo dos conjuntos $\Omega$-limite - parte I . . . . . . . . . . . . . . 41

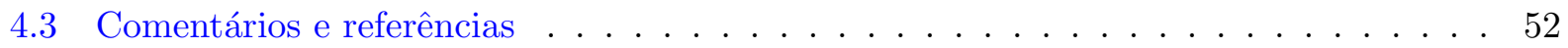

5 Trajetórias regulares $\quad \mathbf{5 5}$

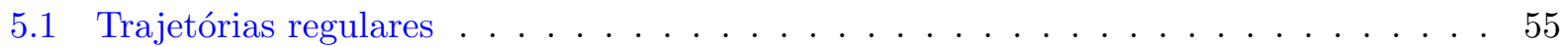

5.2 Estudo dos conjuntos $\Omega$-limite - parte II . . . . . . . . . . . . . . . . 57

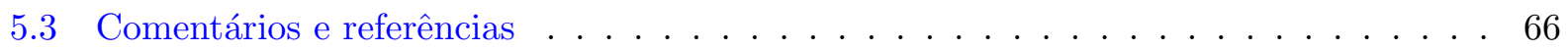

A Construção de uma trajetória de referência regular $\quad 69$

A.1 Construindo uma trajetória de referência periódica . . . . . . . . . . . . . 69

A.2 Famílias regulares . . . . . . . . . . . . . . . . . . . . . 74 


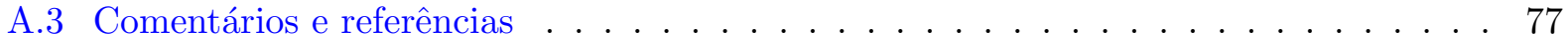

$\begin{array}{ll}\text { Lista de Notações } & 79\end{array}$

$\begin{array}{lr}\text { Referências Bibliográficas } & 81\end{array}$

$\begin{array}{ll}\text { Índice Remissivo } & 82\end{array}$ 


\section{Capítulo 1}

\section{Famílias de campos vetoriais}

Neste capítulo introduzimos as notações da teoria de variedades suaves que empregaremos no decorrer deste trabalho. Exploramos também os fundamentos da análise de famílias de campos vetoriais, enunciando o Teorema da Órbita (Teorema 1.3) e deduzindo suas principais consequências.

\subsection{Notações da teoria de variedades suaves}

No presente trabalho, suave é sinônimo de de classe $C^{\infty}$, seja este adjetivo aplicado a variedades, funções, campos vetoriais, etc. Ainda, variedade - sempre real, com dimensão finita e sem bordo - será sinônimo de variedade suave, ou seja, um conjunto munido de uma estrutura suave tal que a topologia induzida por esta estrutura é Hausdorff e tem base enumerável. Neste caso, indicamos por $C^{\infty}(M)$ a álgebra das funções suaves de $M$ em $\mathbb{R}$.

Dada uma variedade $M$ indicamos por $T_{x} M$ o espaço tangente a $M$ no ponto $x \in M$, o qual é, por definição, o conjunto das derivações de $C^{\infty}(M)$ no ponto $x$. Denotamos ainda por

$$
T M \doteq \bigcup_{x \in M}\{x\} \times T_{x} M
$$

o fibrado tangente de $M$, o qual consideraremos sempre munido de sua estrutura canônica de variedade, induzida pela estrutura suave de $M$. Se $N$ for outra variedade e $\phi: M \rightarrow N$ for suave, definimos seu diferencial no ponto $x \in M$ como sendo a transformação linear $d \phi_{x}: T_{x} M \rightarrow T_{\phi(x)} N$ dada por

$$
\left(d \phi_{x} v\right)(f) \doteq v(f \circ \phi)
$$

para quaisquer $f \in C^{\infty}(N)$ e $v \in T_{x} M$. Este define a aplicação derivada entre os respectivos fibrados tangentes

$$
\begin{aligned}
& d \phi: T M \quad \longrightarrow \quad T N \\
& (x, v) \longmapsto\left(\phi(x), d \phi_{x} v\right)
\end{aligned}
$$

a qual é ela própria uma aplicação suave. No caso particular em que $\phi$ é um difeomorfismo temos 
que $d \phi$ é também um difeomorfismo entre os fibrados tangentes correspondentes e vale a igualdade

$$
d\left(\phi^{-1}\right)=(d \phi)^{-1}
$$

Denotaremos por Diff $M$ o grupo dos difeomorfismos de $M$ em $M$.

Para nós, um campo vetorial em $M$ (não necessariamente suave) é qualquer aplicação $X: M \rightarrow$ $T M$ tal que $\pi \circ X$ é a aplicação identidade de $M$, onde $\pi: T M \rightarrow M$ é a projeção canônica do fibrado tangente. Consideramos o conjunto de todos os campos vetoriais em $M$ munido de sua estrutura canônica de espaço vetorial, induzida por aquela de cada espaço tangente. Quanto aos campos vetoriais suaves, identificaremos estes com as derivações de $C^{\infty}(M)$ : se $X: M \rightarrow T M$ é um campo suave definimos

$$
X: C^{\infty}(M) \rightarrow C^{\infty}(M)
$$

por

$$
X f(x) \doteq X(x) f
$$

para quaisquer $x \in M$ e $f \in C^{\infty}(M)$. Verifica-se que a identificação acima é de fato um isomorfismo linear entre o subespaço vetorial dos campos suaves em $M$ e o espaço das derivações de $C^{\infty}(M)$.

Denotaremos o conjunto dos campos vetoriais suaves em $M$ - sejam eles pensados como seções de $T M$ ou como derivações - por $\operatorname{Vec} M$. Munido do colchete de Lie

$$
[X, Y] \doteq X \circ Y-Y \circ X
$$

definido para $X, Y \in \operatorname{Vec} M$, este conjunto torna-se uma álgebra de Lie real.

Dados $X: M \rightarrow T M$ um campo vetorial e $\phi: M \rightarrow N$ um difeomorfismo, definimos o pushforward de $X$ por $\phi$ como

$$
\phi_{*} X \doteq d \phi \circ X \circ \phi^{-1}
$$

o qual é um campo vetorial em $N$. Note que, sendo $\phi$ um difeomorfismo, $X \in \operatorname{Vec} M$ se e somente se $\phi_{*} X \in \operatorname{Vec} N$, de modo que o pushforward determina uma transformação $\phi_{*}: \operatorname{Vec} M \rightarrow \operatorname{Vec} N$, a qual é um isomorfismo linear. Mais que isso, $\phi_{*}$ é um isomorfismo de álgebras de Lie.

A título de simplicidade, trabalharemos apenas com campos suaves completos, ou seja, aqueles cujas curvas integrais estejam definidas em $\mathbb{R}^{1}$

Dado $X \in \operatorname{Vec} M$, indicaremos por $\left\{\mathbf{e}^{t X}\right\}_{t \in \mathbb{R}}$ o subgrupo a um parâmetro suave gerado por $X$, ou seja:

\footnotetext{
${ }^{1}$ Sem muita perda de generalidade, visto que queremos, em última instância, estudar campos invariantes em grupos de Lie, os quais são sempre completos.
} 
- a aplicação

$$
t \in \mathbb{R} \mapsto \mathbf{e}^{t X} \in \operatorname{Diff} M
$$

é um homomorfismo de grupos;

- a aplicação

$$
(t, x) \in \mathbb{R} \times M \mapsto \mathbf{e}^{t X}(x) \in M
$$

(denominada fluxo do campo vetorial $X$ ) é suave;

- para cada $x \in M$ a aplicação

$$
t \in \mathbb{R} \mapsto \mathbf{e}^{t X}(x) \in M
$$

é uma curva integral de $X$.

Como última observação desta seção, antes de começarmos o assunto principal deste capítulo, vamos recordar como difeomorfismos conjugam as curvas integrais de um campo.

Proposição 1.1. Sejam $X \in \operatorname{Vec} M$ e $\phi \in$ DiffM. Se $Y \doteq \phi_{*} X$ então

$$
\mathbf{e}^{t Y}=\phi \circ \mathbf{e}^{t X} \circ \phi^{-1}
$$

para todo $t \in \mathbb{R}$.

Demonstração. Basta observar que em $t=0$ ambos os operadores são a identidade de $M$ e que, ademais, para cada $t_{0} \in \mathbb{R}$ temos que

$$
\begin{aligned}
\left.\frac{d}{d t}\right|_{t=t_{0}}\left(\phi \circ \mathbf{e}^{t X} \circ \phi^{-1}\right) & =\left.d \phi \circ \frac{d}{d t}\right|_{t=t_{0}}\left(\mathbf{e}^{t X} \circ \phi^{-1}\right) \\
& =d \phi \circ X \circ \mathbf{e}^{t_{0} X} \circ \phi^{-1} \\
& =\left(d \phi \circ X \circ \phi^{-1}\right) \circ\left(\phi \circ \mathbf{e}^{t_{0} X} \circ \phi^{-1}\right) \\
& =Y \circ\left(\phi \circ \mathbf{e}^{t_{0} X} \circ \phi^{-1}\right) .
\end{aligned}
$$

A conclusão segue do teorema de unicidade para curvas integrais.

\subsection{O Teorema da Órbita}

Vamos nesta seção introduzir os conceitos a respeito de famílias de campos vetoriais suaves, necessários para enunciarmos o Teorema da Órbita.

Sejam $M$ uma variedade e $\Gamma \subset \operatorname{Vec} M$ uma família não vazia. Denotamos por 
1. Lie( $(\Gamma)$ a menor subálgebra de Lie de $\operatorname{Vec} M$ que contém $\Gamma$. Não é difícil mostrar que

$$
\operatorname{Lie}(\Gamma)=\operatorname{span} \bigcup_{m \in \mathbb{N}}\left\{\left[X_{1},\left[\ldots\left[X_{m-1}, X_{m}\right] \ldots\right]\right] ; X_{j} \in \Gamma, 1 \leq j \leq m\right\}
$$

2. $\operatorname{Gr}(\Gamma)$ o subgrupo de $\operatorname{Diff} M$ gerado por

$$
\left\{\mathbf{e}^{t X} ; X \in \Gamma, t \in \mathbb{R}\right\}
$$

Nesse contexto definimos, para cada $x \in M$,

$$
\begin{aligned}
& \operatorname{Lie}_{x}(\Gamma) \doteq\{X(x) ; X \in \operatorname{Lie}(\Gamma)\} \subset T_{x} M, \\
& \mathcal{O}_{\Gamma}(x) \doteq\{\phi(x) ; \phi \in \operatorname{Gr}(\Gamma)\} \subset M .
\end{aligned}
$$

O segundo conjunto acima é chamado de órbita de $\Gamma$ a partir de $x$. Considerando a ação natural de $\operatorname{Gr}(\Gamma)$ em $M$ temos que $\mathcal{O}_{\Gamma}(x)$ é exatamente a órbita de $\operatorname{Gr}(\Gamma)$ que contém $x$. Desta última observação segue imediatamente a seguinte

Proposição 1.2. Seja $\Gamma \subset V e c M$. A relação

$$
x \sim y \Leftrightarrow x \in \mathcal{O}_{\Gamma}(y)
$$

é uma relação de equivalência em $M$. Em particular, as órbitas de $\Gamma$ formam uma partição de $M$.

Observe que, dado $x \in M$, podemos descrever sua órbita mais explicitamente como

$$
\mathcal{O}_{\Gamma}(x)=\bigcup_{m \in \mathbb{N}}\left\{\mathbf{e}^{t_{1} X_{1}} \circ \cdots \circ \mathbf{e}^{t_{m} X_{m}}(x) ; t_{j} \in \mathbb{R}, X_{j} \in \Gamma, 1 \leq j \leq m\right\}
$$

Já estamos em condições de enunciar o Teorema da Órbita, cuja demonstração omitimos por fugir do escopo do presente trabalho.

Teorema 1.3 (da Órbita). Sejam $\Gamma \subset V e c M$ e $x_{0} \in M$. Então $\mathcal{O}_{\Gamma}\left(x_{0}\right)$ é um subconjunto conexo de $M$ que admite uma estrutura de subvariedade imersa. Com essa estrutura temos que

$$
T_{x} \mathcal{O}_{\Gamma}\left(x_{0}\right)=\operatorname{span}\left\{\phi_{*} X(x) ; \phi \in G r(\Gamma), X \in \Gamma\right\}
$$

para todo $x \in \mathcal{O}_{\Gamma}\left(x_{0}\right)$.

Gostaríamos de definir, para cada $x \in M$,

$$
D_{x} \doteq \operatorname{span}\left\{\phi_{*} X(x) ; \phi \in \operatorname{Gr}(\Gamma), X \in \Gamma\right\}
$$


e alegar que

$$
D \doteq \bigcup_{x \in M}\{x\} \times D_{x} \subset T M
$$

é uma distribuição suave no sentido clássico, a qual possui as órbitas de $\Gamma$ como variedades integrais. Infelizmente o raciocínio acima falha pois, em princípio, a dimensão de $D_{x}$ pode variar, mesmo localmente, de modo que $D$ não é uma distribuição no sentido tradicional. Na única aplicação em que utilizaremos um argumento deste tipo - a interpretação do Teorema da Órbita em grupos de Lie (Teorema 2.12) - isto não constituirá um problema, já que no contexto de grupos de Lie esta dificuldade desaparece: teremos simetrias suficientes para garantir a invariância da dimensão de $D_{x}$ (Lema 2.11).

\subsection{Famílias Lie-determinadas}

Recordemos que se $\Gamma \subset$ Vec $M$ é uma família suave então $\operatorname{Lie}_{x}(\Gamma)$ é um subespaço vetorial de $T_{x} M$ para cada $x \in M$. É uma consequência imediata do Teorema da Órbita que, mais que isso, vale a seguinte inclusão.

Proposição 1.4. Fixado $x_{0} \in M$ temos

$$
\operatorname{Lie}_{x}(\Gamma) \subset T_{x} \mathcal{O}_{\Gamma}\left(x_{0}\right)
$$

para todo $x \in \mathcal{O}_{\Gamma}\left(x_{0}\right)$.

Demonstração. Pelo Teorema da Órbita temos, para cada $x \in \mathcal{O}_{\Gamma}\left(x_{0}\right)$, que

$$
T_{x} \mathcal{O}_{\Gamma}\left(x_{0}\right)=\operatorname{span}\left\{\phi_{*} X(x) ; \phi \in \operatorname{Gr}(\Gamma), X \in \Gamma\right\}
$$

de modo que, em particular,

$$
X(x) \in T_{x} \mathcal{O}_{\Gamma}\left(x_{0}\right)
$$

para todo $x \in \mathcal{O}_{\Gamma}\left(x_{0}\right)$ e para todo $X \in \Gamma$. Ou seja, os elementos de $\Gamma$ são tangentes a $\mathcal{O}_{\Gamma}\left(x_{0}\right)$. Mas, como é fato bem conhecido, se dois campos suaves são tangentes a uma subvariedade imersa então seu colchete de Lie também o é. Isso implica que todo elemento de $\operatorname{Lie}(\Gamma)$ é tangente a $\mathcal{O}_{\Gamma}\left(x_{0}\right)$ e então temos a inclusão proposta em cada ponto.

Em geral, a inclusão acima é estrita i.e. $\operatorname{Lie}_{x}(\Gamma) \neq T_{x} \mathcal{O}_{\Gamma}\left(x_{0}\right)$. São conhecidas diversas classes especiais de famílias suaves para as quais a inclusão acima é, de fato, uma igualdade. No presente trabalho, porém, apresentaremos apenas um critério muito simples, mas que será suficiente para uma aplicação no estudo de famílias invariantes em grupos de Lie. 
Lema 1.5. Seja $\mathcal{V} \subset V e c M$ um subespaço vetorial de dimensão finita e suponha que $X \in V e c M$ é tal que

$$
[X, V] \in \mathcal{V} \text { para todo } V \in \mathcal{V}
$$

Então $\left(\mathbf{e}^{t X}\right)_{*} V \in \mathcal{V}$ para todo $t \in \mathbb{R}$ e para todo $V \in \mathcal{V}$

Demonstração. Seja $V_{1}, \ldots, V_{m} \in \mathcal{V}$ uma base vetorial de $\mathcal{V}$. Como $\mathcal{V}$ é um subespaço vetorial é suficiente mostrar que $\left(\mathbf{e}^{t X}\right)_{*} V_{j} \in \mathcal{V}$ para cada $j=1, \ldots, m$. Por hipótese, para cada $j=1, \ldots, m$, temos que $\left[X, V_{j}\right] \in \mathcal{V}$ e, portanto, existem coeficientes $a_{j k} \in \mathbb{R}, 1 \leq k \leq m$, tais que

$$
\left[X, V_{j}\right]=\sum_{k=1}^{m} a_{j k} V_{k}
$$

Contudo, para cada $x \in M$ fixado temos que

$$
\left[X, V_{j}\right](x)=\left.\frac{d}{d t}\right|_{t=0}\left(\mathbf{e}^{-t X}\right)_{*} V_{j}(x)
$$

(é simplesmente a derivada de Lie de $V_{j}$ com relação a $X$ ), de modo que, se definirmos $v_{j}: \mathbb{R} \rightarrow T_{x} M$ por

$$
v_{j}(t) \doteq\left(\mathbf{e}^{-t X}\right)_{*} V_{j}(x)
$$

para cada $j=1, \ldots, m$, temos que

$$
v_{j}^{\prime}(0)=\left.\frac{d}{d t}\right|_{t=0}\left(\mathbf{e}^{-t X}\right)_{*} V_{j}(x)=\left[X, V_{j}\right](x)
$$

e para $t_{0} \in \mathbb{R}$ temos

$$
\begin{aligned}
v_{j}^{\prime}\left(t_{0}\right) & =\left.\frac{d}{d t}\right|_{t=0} v_{j}\left(t_{0}+t\right) \\
& =\left.\frac{d}{d t}\right|_{t=0}\left(\mathbf{e}^{-\left(t_{0}+t\right) X}\right)_{*} V_{j}(x) \\
& =\left.\frac{d}{d t}\right|_{t=0}\left(\mathbf{e}^{-t X}\right)_{*}\left\{\left(\mathbf{e}^{-t_{0} X}\right)_{*} V_{j}\right\}(x) \\
& =\left[X,\left(\mathbf{e}^{-t_{0} X}\right)_{*} V_{j}\right](x) \\
& =\left[\left(\mathbf{e}^{-t_{0} X}\right)_{*} X,\left(\mathbf{e}^{-t_{0} X}\right)_{*} V_{j}\right](x) \\
& =\left(\mathbf{e}^{-t_{0} X}\right)_{*}\left[X, V_{j}\right](x)
\end{aligned}
$$

onde usamos novamente a derivada de Lie na quarta igualdade e, na penúltima igualdade, usamos que $\left(\mathbf{e}^{t X}\right)_{*} X=X$ para todo $t \in \mathbb{R}$ e para todo $X \in$ Vec $M$ (o que é facilmente verificado utilizando 
a Proposição 1.1 e comparando os fluxos). Concluímos que

$$
v_{j}^{\prime}(t)=\left(\mathbf{e}^{-t X}\right)_{*}\left[X, V_{j}\right](x)
$$

para todo $t \in \mathbb{R}$. Mas, recordado que $\left[X, V_{j}\right]$ é combinação linear de $V_{1}, \ldots, V_{m}$ temos

$$
\begin{aligned}
v_{j}^{\prime}(t) & =\left(\mathbf{e}^{-t X}\right)_{*}\left(\sum_{k=1}^{m} a_{j k} V_{k}\right)(x) \\
& =\sum_{k=1}^{m} a_{j k}\left(\mathbf{e}^{-t X}\right)_{*} V_{k}(x) \\
& =\sum_{k=1}^{m} a_{j k} v_{k}(t)
\end{aligned}
$$

conforme a nossa definição de $v_{k}$. Ou seja

$$
v_{j}^{\prime}=\sum_{k=1}^{m} a_{j k} v_{k}, 1 \leq j \leq m,
$$

que é uma EDO linear autônoma em $T_{x} M$, com condições iniciais

$$
v_{j}(0)=V_{j}(x), 1 \leq j \leq m .
$$

Logo, existem funções $\alpha_{j k} \in C^{\infty}(\mathbb{R})$ tais que

$$
v_{j}(t)=\sum_{k=1}^{m} \alpha_{j k}(t) V_{k}(x)
$$

para todo $t \in \mathbb{R}$ e $j=1, \ldots, m$ (mais especificamente, $\left(\alpha_{j k}(t)\right)_{1 \leq j, k \leq m}=\mathbf{e}^{t A}$ é a exponencial da matriz $\left.A=\left(a_{j k}\right)_{1 \leq j, k \leq m}\right)$. Substituindo $v_{j}(t)=\left(\mathbf{e}^{-t X}\right)_{*} V_{j}(x)$ na igualdade acima temos

$$
\left(\mathbf{e}^{-t X}\right)_{*} V_{j}(x)=\sum_{k=1}^{m} \alpha_{j k}(t) V_{k}(x)
$$

e, como $x \in M$ é arbitrário,

$$
\left(\mathbf{e}^{-t X}\right)_{*} V_{j}=\sum_{k=1}^{m} \alpha_{j k}(t) V_{k}
$$

que é um elemento de $\mathcal{V}$ para todo $t \in \mathbb{R}$.

Proposição 1.6. Seja $\Gamma \subset V e c M$ uma família suave tal que Lie $(\Gamma)$ é um subespaço de dimensão 
finita de VecM. Neste caso, dado $x_{0} \in M$ temos que

$$
\operatorname{Lie}_{x}(\Gamma)=T_{x} \mathcal{O}_{\Gamma}\left(x_{0}\right)
$$

para todo $x \in \mathcal{O}_{\Gamma}\left(x_{0}\right)$.

Demonstração. Vamos provar que

$$
\phi_{*} X \in \operatorname{Lie}(\Gamma)
$$

para quaisquer $\phi \in \operatorname{Gr}(\Gamma)$ e $X \in \Gamma$. De fato, como $\operatorname{Lie}(\Gamma)$ tem dimensão finita, segue do lema anterior que

$$
\left(\mathbf{e}^{t Y}\right)_{*} V \in \operatorname{Lie}(\Gamma)
$$

para quaisquer $t \in \mathbb{R}, Y \in \Gamma$ e $V \in \operatorname{Lie}(\Gamma)$ pois, nesse caso, $Y \in \operatorname{Lie}(\Gamma)$ e, $\operatorname{logo},[Y, V] \in \operatorname{Lie}(\Gamma)$. Como todo $\phi \in \operatorname{Gr}(\Gamma)$ pode ser escrito como

$$
\phi=\mathbf{e}^{t_{1} Y_{1}} \circ \cdots \circ \mathbf{e}^{t_{m} Y_{m}}
$$

onde $t_{1}, \ldots, t_{m} \in \mathbb{R}$ e $Y_{1}, \ldots, Y_{m} \in \Gamma$ temos, para $X \in \Gamma$, que

$$
\phi_{*} X=\left(\mathbf{e}^{t_{1} Y_{1}}\right)_{*} \cdots\left(\mathbf{e}^{t_{m} Y_{m}}\right)_{*} X
$$

é um elemento de $\operatorname{Lie}(\Gamma)$, conforme prometido.

Assim, em um ponto $x \in \mathcal{O}_{\Gamma}\left(x_{0}\right)$ temos

$$
\phi_{*} X(x) \in \operatorname{Lie}_{x}(\Gamma)
$$

para quaisquer $\phi \in \operatorname{Gr}(\Gamma)$ e $X \in \Gamma$. Logo

$$
\operatorname{span}\left\{\phi_{*} X(x) ; \phi \in \operatorname{Gr}(\Gamma), X \in \Gamma\right\} \subset \operatorname{Lie}_{x}(\Gamma)
$$

ou seja

$$
T_{x} \mathcal{O}_{\Gamma}\left(x_{0}\right) \subset \operatorname{Lie}_{x}(\Gamma) .
$$

Esta era a inclusão que faltava: a outra era o conteúdo da Proposição 1.4.

A discussão acima motiva a definição de família Lie-determinada.

Definição 1.7. Uma família $\Gamma \subset \operatorname{Vec} M$ é dita Lie-determinada se $\operatorname{Lie}_{x}(\Gamma)=T_{x} M$ para todo $x \in M$. 
Teorema 1.8 (Rashevsky-Chow). Seja $\Gamma \subset$ VecM uma familia suave. Se

$$
\operatorname{Lie}_{x}(\Gamma)=T_{x} M
$$

para algum $x \in M$ então $\mathcal{O}_{\Gamma}(x)$ é uma subvariedade aberta.

Em particular, se $M$ é uma variedade conexa e $\Gamma \subset$ VecM é uma família Lie-determinada então

$$
\mathcal{O}_{\Gamma}(x)=M
$$

para todo $x \in M$.

Demonstração. De acordo com o Teorema da Órbita temos que $\mathcal{O}_{\Gamma}(x)$ é uma subvariedade imersa de $M$. Segundo a Proposição 1.4 temos

$$
\operatorname{Lie}_{x}(\Gamma) \subset T_{x} \mathcal{O}_{\Gamma}(x) \subset T_{x} M
$$

o que, juntamente com a hipótese de que $\operatorname{Lie}_{x}(\Gamma)=T_{x} M$, implica que

$$
T_{x} \mathcal{O}_{\Gamma}(x)=T_{x} M
$$

Isso implica que $\operatorname{dim} \mathcal{O}_{\Gamma}(x)=\operatorname{dim} M$ como variedades. Daí temos que

$$
T_{y} \mathcal{O}_{\Gamma}(x)=T_{y} M
$$

para todo $y \in \mathcal{O}_{\Gamma}(x)$. Mas se denotarmos

$$
i: \mathcal{O}_{\Gamma}(x) \rightarrow M
$$

a aplicação de inclusão, a qual, pelo Teorema da Órbita, é uma imersão, temos que

$$
d i_{y}: T_{y} \mathcal{O}_{\Gamma}(x) \rightarrow T_{y} M
$$

é na verdade um isomorfismo linear para todo $y \in \mathcal{O}_{\Gamma}(x)$.

Isto implica que $\mathcal{O}_{\Gamma}(x)$ é uma subvariedade aberta de $M$. Com efeito, fixado $y \in \mathcal{O}_{\Gamma}(x)$ temos, aplicando o Teorema da Função Inversa à aplicação $i$ definida acima, que existe $U \subset \mathcal{O}_{\Gamma}(x)$ aberto na topologia de $\mathcal{O}_{\Gamma}(x)$ que contém y e tal que $i(U)$ é aberto em $M$. Mas $i(U)=U$ (como conjuntos) de modo que $U$ também é aberto em $M$. Daí y é um ponto interior de $\mathcal{O}_{\Gamma}(x)$ na topologia de $M$. Como y é arbitrário, $\mathcal{O}_{\Gamma}(x)$ é um subconjunto aberto de $M$.

Resta mostrar que a estrutura suave de $\mathcal{O}_{\Gamma}(x)$ dada pelo Teorema da Órbita coincide com a estrutura de subvariedade aberta de $M$. Mas, prosseguindo com o argumento acima do Teorema 
da Função Inversa temos que a aplicação identidade

$$
\operatorname{id~}: \mathcal{O}_{\Gamma}(x) \rightarrow \mathcal{O}_{\Gamma}(x)
$$

(onde no domínio consideramos a estrutura de subvariedade imersa e no contradomínio consideramos a estrutura de subvariedade aberta) é um difeomorfismo local em cada ponto. Como ademais é bijetora, temos que a aplicação id acima é um difeomorfismo global. Isso prova que as estruturas suaves acima são iguais e, portanto, $\mathcal{O}_{\Gamma}(x)$ é uma subvariedade aberta de $M$.

Se, em particular, $\Gamma$ é Lie-determinada, temos que $\mathcal{O}_{\Gamma}(x)$ é aberto em $M$ para todo $x \in M$. Mas, como já vimos, $\left\{\mathcal{O}_{\Gamma}(x) ; x \in M\right\}$ é uma partição de $M$ (Proposição 1.2), de modo que, sendo $M$ conexa, existe somente uma órbita, a qual deve ser igual a $M$.

\subsection{Comentários e referências}

A principal referência para este capítulo é [AS04], embora aqui empreguemos uma notação distinta. Uma referência alternativa para os tópicos aqui explorados é [Jur97]. As questões referentes à teoria de variedades suaves podem ser encontradas em qualquer livro introdutório sobre o assunto. Para fins de referência seguiremos exclusivamente [Lee03].

A menos de diferenças de notação, o enunciado do Teorema da Órbita (Teorema 1.3) é exatamente aquele encontrado em [AS04], Theorem 5.1, páginas 63 (enunciado) e 67-70 (demonstração). A principal diferença é que nessa referência os autores descrevem o espaço tangente à órbita utilizando o "formalismo cronológico" de Agrachev e Gamkrelidze. Convertendo para a notação clássica, obtemos exatamente a expressão (1.1), trocando somente o pushforward $\phi_{*}$ pelo pullback $\phi^{*}-\mathrm{o}$ que não faz diferença para a definição do conjunto (1.1), já que $\operatorname{Gr}(\Gamma)$ é um subgrupo de Diff $M$.

O nosso Lema 1.5 é essencialmente uma versão simplificada do resultado encontrado em [AS04], Lemma 5.15, página 72, o qual visa critérios mais abrangentes ligados a famílias localmente finitamente geradas e é uma generalização do que apresentamos aqui. 


\section{Capítulo 2}

\section{Tópicos da Teoria de Lie}

O presente capítulo tem diversos objetivos. Na Seção 2.1 introduzimos os conceitos básicos das álgebras de Lie reais (representação adjunta, forma de Killing, semi-simplicidade) que empregaremos no decorrer do capítulo. Recapitulamos as noções e notações básicas dos grupos de Lie na Seção 2.2, além de (re)demonstrar alguns resultados clássicos utilizando a nossa notação. Na Seção 2.3 demonstramos como a estrutura de grupo de Lie afeta o Teorema da Órbita e seus corolários, simplificando muitos aspectos da análise das famílias de campos invariantes à esquerda. Finalmente, exploramos na Seção 2.4 alguns resultados da teoria dos grupos de Lie compactos que necessitaremos nos capítulos seguintes.

\subsection{Generalidades sobre álgebras de Lie}

Seja $\mathfrak{g}$ uma álgebra de Lie real com dimensão finita. Denotaremos por $[\cdot, \cdot]: \mathfrak{g} \times \mathfrak{g} \rightarrow \mathfrak{g}$ seu colchete, o qual por definição é uma aplicação bilinear antissimétrica que satisfaz a identidade de Jacobi

$$
[X,[Y, Z]]+[Y,[Z, X]]+[Z,[X, Y]]=0
$$

para quaisquer $X, Y, Z \in \mathfrak{g}$.

Para cada $X \in \mathfrak{g}$ definimos

$$
\begin{aligned}
\operatorname{ad}(X) \quad: \quad \mathfrak{g} & \longrightarrow \mathfrak{g} \\
Y & \longmapsto[X, Y]
\end{aligned}
$$

a qual é evidentemente uma transformação linear de $\mathfrak{g}$. Definimos então a representação adjunta de $\mathfrak{g}$ como sendo a aplicação

$$
\begin{aligned}
\operatorname{ad}: \mathfrak{g} & \longrightarrow \mathfrak{g l}(\mathfrak{g}) \\
X & \longmapsto \operatorname{ad}(X)
\end{aligned}
$$

a qual, por sua vez, é um homomorfismo de álgebras de Lie. Não é difícil verificar que se $\phi: \mathfrak{g} \rightarrow \mathfrak{g}$ 
é um isomorfismo de álgebras de Lie vale que

$$
\operatorname{ad}(\phi X)=\phi \cdot \operatorname{ad}(X) \cdot \phi^{-1}
$$

para todo $X \in \mathfrak{g}$.

Definimos a forma de Killing de $\mathfrak{g}$ como

$$
B(X, Y) \doteq \operatorname{tr}\{\operatorname{ad}(X) \cdot \operatorname{ad}(Y)\}
$$

a qual é evidentemente uma forma bilinear simétrica em $\mathfrak{g}$. Também não é difícil mostrar que $B$ é ad-invariante no sentido de que para cada $X \in \mathfrak{g}$ fixado temos

$$
B(Y, \operatorname{ad}(X) Z)=-B(\operatorname{ad}(X) Y, Z)
$$

para quaisquer $Y, Z \in \mathfrak{g}^{1}$.

Dizemos que $B$ é não-degenerada se para todo $X \in \mathfrak{g}$ a condição

$$
B(X, Y)=0 \text { para todo } Y \in \mathfrak{g}
$$

implica que $X=0$.

Definição 2.1. Dizemos que g é semi-simples se $B$ for não-degenerada.

Dizemos que $\mathfrak{i} \subset \mathfrak{g}$ é um ideal se for um subespaço vetorial que satisfaz

$$
X \in \mathfrak{i}, Y \in \mathfrak{g} \Rightarrow[X, Y] \in \mathfrak{i} .
$$

Definição 2.2. Dizemos que $\mathfrak{g}$ é redutiva se para todo ideal $\mathfrak{i} \subset \mathfrak{g}$ existe um ideal $\mathfrak{j} \subset \mathfrak{g}$ tal que

$$
\mathfrak{g}=\mathfrak{i} \oplus \mathfrak{j}
$$

Álgebras de Lie redutivas serão particularmente importantes para nós em razão das propriedades que enunciamos a seguir, e porque toda álgebra de Lie oriunda de um grupo de Lie compacto pertence a esta classe.

Definimos o centro de $\mathfrak{g}$ por

$$
\mathfrak{z}(\mathfrak{g}) \doteq\{X \in \mathfrak{g} ;[X, Y]=0 \text { para todo } Y \in \mathfrak{g}\}
$$

o qual é o núcleo da representação adjunta. Verifica-se que se $\mathfrak{g}$ é semi-simples então $\mathfrak{z}(\mathfrak{g})=\{0\}$.

Teorema 2.3. Se $\mathfrak{g}$ é redutiva então

$$
\mathfrak{g}=\mathfrak{z}(\mathfrak{g}) \oplus \mathfrak{g}^{\prime}
$$

\footnotetext{
${ }^{1}$ [Kna02], Capítulo I, equação (1.19a), página 36.
} 
onde $\mathfrak{g}^{\prime}$ é o menor ideal que contém $[\mathfrak{g}, \mathfrak{g}]$, o qual é semi-simples.

Referência. [Kna02], Corollary 1.56, página 56.

Dessa forma, se $\mathfrak{g}$ é redutiva então vale a recíproca da observação anterior: se $\mathfrak{z}(\mathfrak{g})=\{0\}$ então $\mathfrak{g}$ é semi-simples.

\subsection{Generalidades sobre grupos de Lie}

Seja $G$ um grupo de Lie i.e. um grupo munido de uma estrutura suave tal que as operações de multiplicação e inversão são suaves. Para cada $x \in G$ denotaremos as translações por $x$, à esquerda e à direita, respectivamente, por

$$
\begin{aligned}
L_{x}: G & \longrightarrow G \\
y & \longmapsto x \cdot y \\
R_{x}: G & \longrightarrow G \\
y & \longmapsto y \cdot x
\end{aligned}
$$

as quais são difeomorfismos de $G$. Denotaremos também a identidade de $G$ por $e$.

Nesta seção denotaremos por $\mathfrak{g}$ a álgebra de Lie de $G$, a qual por definição é a subálgebra de Lie de VecG dos campos vetoriais invariantes à esquerda, ou seja, $X \in \mathfrak{g}$ se e somente se

$$
\left(L_{x}\right)_{*} X=X
$$

para todo $x \in G$. É um fato bastante conhecido que todo campo invariante à esquerda é completo.

Frequentemente faremos a identificação

$$
\begin{aligned}
\mathfrak{g} & \longrightarrow T_{e} G \\
X & \longmapsto X(e)
\end{aligned}
$$

a qual é um isomorfismo linear e induz em $T_{e} G$ uma única estrutura de álgebra de Lie que torna a identificação acima um isomorfismo de álgebras de Lie.

A seguinte proposição motiva a definição da aplicação exponencial.

Proposição 2.4. Se $X \in \mathfrak{g}$ então

$$
\mathbf{e}^{t X}=R_{\mathbf{e}^{t X}(e)}
$$

para todo $t \in \mathbb{R}$. Em particular, $\mathbf{e}^{t X}$ comuta com qualquer translação à esquerda.

Demonstração. Como $X$ é invariante à esquerda temos $X=\left(L_{x}\right)_{*} X$ para cada $x \in G$. Segue então da Proposição 1.1 que, para todo $t \in \mathbb{R}$, temos

$$
\mathbf{e}^{t X}=L_{x} \circ \mathbf{e}^{t X} \circ L_{x}^{-1}
$$


de modo que, calculando os dois lados da igualdade acima exatamente no ponto $x$

$$
\mathbf{e}^{t X}(x)=L_{x} \circ \mathbf{e}^{t X} \circ L_{x}^{-1}(x)=x \cdot \mathbf{e}^{t X}(e)
$$

e como $x \in G$ é arbitrário nesta última igualdade, concluímos que $\mathbf{e}^{t X}=R_{\mathbf{e}^{t X}(e)}$.

É um fato de simples verificação que uma translação à direita comuta com qualquer translação à esquerda. Esta observação e a conclusão acima encerram a demonstração.

Motivados pela proposição acima, daqui para frente - e, claro, somente no contexto de grupos de Lie - usaremos a notação $\mathbf{e}^{t X}$ tanto para indicar o difeomorfismo

$$
x \in G \mapsto x \cdot \mathbf{e}^{t X}(e) \in G
$$

quanto o próprio elemento $\mathbf{e}^{t X}(e) \in G$ : a interpretação correta deverá ser deduzida do contexto.

Definição 2.5. Chamamos a aplicação

$$
\begin{aligned}
\mathbf{e}: \mathfrak{g} & \longrightarrow G \\
X & \longmapsto \mathbf{e}^{X}
\end{aligned}
$$

de aplicação exponencial de $G$.

Dado $x \in G$ definimos a conjugação por $x$ como a transformação

$$
\begin{aligned}
I_{x}: G & \longrightarrow \\
y & \longmapsto x \cdot y \cdot x^{-1}
\end{aligned}
$$

ou, em uma notação mais concisa,

$$
I_{x}=R_{x^{-1}} \circ L_{x}=L_{x} \circ R_{x^{-1}}
$$

É evidente, a partir da última relação acima, que $I_{x}$ é um difeomorfismo. Um conta simples revela que $I_{x}$ é também um homomorfismo de $G$ em $G$, ou seja, $I_{x}$ é um automorfismo de $G$. Observe ainda que a aplicação

$$
x \in G \mapsto I_{x} \in \operatorname{Diff} G
$$

é um homomorfismo de grupos.

Para cada $x \in G$ definimos a transformação $\operatorname{Ad}(x): T_{e} G \rightarrow T_{e} G$ por

$$
\operatorname{Ad}(x) \doteq d\left(I_{x}\right)_{e}
$$

a qual é um isomorfismo linear de $T_{e} G$ em $T_{e} G$, visto que $I_{x}$ é um difeomorfismo. Se $X \in \mathfrak{g}$ é um 
campo invariante à esquerda segue facilmente da definição de $\operatorname{Ad}(x)$ que

$$
\operatorname{Ad}(x) X(e)=\left(I_{x}\right)_{*} X(e)
$$

ou seja, podemos pensar em $\operatorname{Ad}(x)$ como uma transformação linear de $\mathfrak{g}$ em $\mathfrak{g}$ dada por

$$
\operatorname{Ad}(x)=\left(I_{x}\right)_{*}
$$

a qual é obviamente inversível. Em particular, $\operatorname{Ad}(x): \mathfrak{g} \rightarrow \mathfrak{g}$ é um isomorfismo de álgebras de Lie (pois é o pushforward de um difeomorfismo). Usando ainda a relação (2.2) temos, para cada $X \in \mathfrak{g}$, que

$$
\operatorname{Ad}(x) X=\left(R_{x^{-1}}\right)_{*}\left(L_{x}\right)_{*} X=\left(R_{x^{-1}}\right)_{*} X
$$

visto que $X$ é invariante à esquerda. Logo podemos escrever a expressão mais simples

$$
\operatorname{Ad}(x)=\left(R_{x^{-1}}\right)_{*} .
$$

Definição 2.6. A aplicação

$$
\mathrm{Ad}: G \rightarrow \mathrm{GL}(\mathfrak{g})
$$

definida acima é chamada de representação adjunta de $G$.

É fácil verificar que a representação adjunta (2.3) é um homomorfismo de grupos. Mais, é possível demonstrar que ela é suave ${ }^{2}$. Identificando canonicamente

$$
\begin{aligned}
T_{e} G & \cong \mathfrak{g} \\
T_{\mathrm{id}} \mathrm{GL}(\mathfrak{g}) & \cong \mathfrak{g l}(\mathfrak{g})
\end{aligned}
$$

podemos pensar no diferencial

$$
d \mathrm{Ad}_{e}: T_{e} G \rightarrow T_{\mathrm{id}} \mathrm{GL}(\mathfrak{g})
$$

como uma transformação linear de $\mathfrak{g}$ em $\mathfrak{g l}(\mathfrak{g})$. Com essas identificações, é possível mostrar ${ }^{3}$ que

$$
d \operatorname{Ad}_{e} X=\operatorname{ad}(X)
$$

onde ad é a representação adjunta de $\mathfrak{g}$ definida na Seção 2.1. Analogamente, podemos pensar em

2 [Lee03], página 211.

3 [Lee03], Theorem 20.12, páginas 529 e 530. 
ad com domínio em $T_{e} G$ pela identificação

$$
\operatorname{ad}(X(e)) \doteq \operatorname{ad}(X)
$$

para qualquer $X \in \mathfrak{g}$, onde o lado direito já está definido.

Com a finalidade de simplificar algumas contas que surgirão no decorrer do presente trabalho, será necessária uma expressão explícita - envolvendo a representação adjunta de $\mathfrak{g}$ - do diferencial de Ad em pontos arbitrários de $G$, a qual obtemos por translações.

Proposição 2.7. Para qualquer $(x, v) \in T G$ temos que

$$
d A d_{x} v=A d(x) \cdot a d\left(d L_{x^{-1}} v\right) .
$$

Em particular, se $X \in \mathfrak{g}$ temos que

$$
d A d X(x)=A d(x) \cdot \operatorname{ad}(X) \text { para todo } x \in G
$$

Demonstração. Fixado $(x, v) \in T G$ observamos, inicialmente, que

$$
v \in T_{x} G \Rightarrow d L_{x^{-1}} v \in T_{e} G
$$

de modo que sabemos calcular ad $\left(d L_{x^{-1}} v\right)$ conforme a equação (2.4) e, portanto, os termos apresentados na igualdade (2.5) estão bem definidos.

A fim de demonstrar (2.5), considere a aplicação $F \doteq \operatorname{Ad} \circ L_{x}$ de modo que $\mathrm{Ad}=F \circ L_{x^{-1}}$. Pela regra da cadeia

$$
d \operatorname{Ad}_{x} v=d F_{e} d L_{x^{-1}} v
$$

de modo que para demonstrar (2.5) é suficiente computar $d F_{e}$. Para tal, observe que também podemos escrever

$$
F(y)=\operatorname{Ad}(x \cdot y)=\operatorname{Ad}(x) \cdot \operatorname{Ad}(y)
$$

para todo $y \in G$, ou seja, $F=\Lambda \circ$ Ad onde

$$
\begin{aligned}
\Lambda: \mathfrak{g l}(\mathfrak{g}) & \longrightarrow \mathfrak{g l}(\mathfrak{g}) \\
T & \longmapsto \operatorname{Ad}(x) \cdot T
\end{aligned}
$$

é uma transformação linear. Assim, se $\xi \in T_{e} G$ temos novamente pela regra da cadeia

$$
\begin{aligned}
d F_{e} \xi & =d \Lambda_{\operatorname{Ad}(e)} d \operatorname{Ad}_{e} \xi \\
& =\Lambda(\operatorname{ad}(\xi)) \\
& =\operatorname{Ad}(x) \cdot \operatorname{ad}(\xi) .
\end{aligned}
$$


Em particular se tomarmos $\xi \doteq d L_{x^{-1}} v$ temos que

$$
\begin{aligned}
d \operatorname{Ad}_{x} v & =d F_{e} d L_{x^{-1}} v \\
& =\operatorname{Ad}(x) \cdot \operatorname{ad}\left(d L_{x^{-1}} v\right)
\end{aligned}
$$

o que prova a identidade (2.5).

Tomando agora $X \in \mathfrak{g}$ temos, escolhendo $v \doteq X(x)$ na igualdade anterior, que

$$
\begin{aligned}
d \operatorname{Ad} X(x) & =\operatorname{Ad}(x) \cdot \operatorname{ad}\left(d L_{x^{-1}} X(x)\right) \\
& =\operatorname{Ad}(x) \cdot \operatorname{ad}\left(X\left(L_{x^{-1}} x\right)\right) \\
& =\operatorname{Ad}(x) \cdot \operatorname{ad}(X(e)) \\
& =\operatorname{Ad}(x) \cdot \operatorname{ad}(X) .
\end{aligned}
$$

Para cada $x \in G$, como $\operatorname{Ad}(x): \mathfrak{g} \rightarrow \mathfrak{g}$ é um isomorfismo de álgebras de Lie temos, como caso particular de (2.1), que

$$
\operatorname{ad}(\operatorname{Ad}(x) X)=\operatorname{Ad}(x) \cdot \operatorname{ad}(X) \cdot \operatorname{Ad}(x)^{-1}
$$

para todo $X \in \mathfrak{g}$.

Finalmente, a relação entre a representação adjunta e a aplicação exponencial é dada por ${ }^{4}$

$$
\operatorname{Ad}\left(\mathbf{e}^{X}\right)=\mathbf{e}^{\operatorname{ad}(X)}
$$

para todo $X \in \mathfrak{g}$.

\subsection{O Teorema da Órbita em grupos de Lie}

Nesta seção ainda consideramos $G$ um grupo de Lie e g sua álgebra de Lie. Da Proposição 2.4 segue por indução que

Corolário 2.8. Se $X_{1}, \ldots, X_{m} \in \mathfrak{g} e t_{1}, \ldots, t_{m} \in \mathbb{R}$ então

$$
\mathbf{e}^{t_{1} X_{1}} \circ \cdots \circ \mathbf{e}^{t_{m} X_{m}}(x)=x \cdot \mathbf{e}^{t_{m} X_{m}} \cdot \ldots \cdot \mathbf{e}^{t_{1} X_{1}}
$$

para todo $x \in G$.

Em particular, se $\Gamma \subset \mathfrak{g}$ e $\phi \in G r(\Gamma)$ então

$$
\phi(x)=x \cdot \phi(e)
$$

para todo $x \in G$, ou seja, $\phi=R_{\phi(e)}$.

\footnotetext{
${ }^{4}$ Veja, por exemplo, [Lee03], demonstração do Theorem 20.19, equação (20.14), página 536.
} 
No decorrer deste trabalho, família invariante à esquerda será sinônimo de família de campos vetoriais invariantes à esquerda, ou seja, um subconjunto da álgebra de Lie g. O último corolário acima implica uma caracterização muito mais simples para as órbitas destas famílias.

Proposição 2.9. Seja $\Gamma \subset \mathfrak{g}$ uma família invariante à esquerda. Então $\mathcal{O}_{\Gamma}(e)$ é um subgrupo de G. Ademais

$$
\mathcal{O}_{\Gamma}(x)=x \cdot \mathcal{O}_{\Gamma}(e)
$$

para todo $x \in G$.

Demonstração. Basta observar que a aplicação

$$
\phi \in \operatorname{Gr}(\Gamma) \mapsto \phi(e) \in G
$$

é um anti-homomorfismo de grupos. De fato, pelo corolário anterior, se $\phi_{1}, \phi_{2} \in \operatorname{Gr}(\Gamma)$ então

$$
\left(\phi_{1} \circ \phi_{2}\right)(e)=\phi_{1}\left(\phi_{2}(e)\right)=\phi_{2}(e) \cdot \phi_{1}(e)
$$

Desse modo

$$
\mathcal{O}_{\Gamma}(e)=\{\phi(e) ; \phi \in \operatorname{Gr}(\Gamma)\}
$$

é um subgrupo de $G$.

A proposição anterior permite reduzir o estudo das órbitas de uma família invariante à esquerda estritamente ao estudo da órbita que contém a identidade do grupo. O que faremos daqui para frente é um estudo sistemático desse conjunto, o qual denotaremos

$$
\mathcal{O}_{\Gamma} \doteq \mathcal{O}_{\Gamma}(e)
$$

e denominaremos a órbita de $\Gamma$.

Podemos agora interpretar o Teorema da Órbita no caso particular de famílias invariantes à esquerda em grupos de Lie. Já sabemos que $\mathcal{O}_{\Gamma}$ possui uma estrutura de subvariedade imersa dada pelo Teorema 1.3. Ademais, acabamos de provar que $\mathcal{O}_{\Gamma}$ é um subgrupo (abstrato) de $G$. Queremos mostrar que, na verdade, $\mathcal{O}_{\Gamma}$ é um subgrupo de Lie de $G$, e para tanto precisamos de mais alguns resultados preliminares.

Primeiramente definimos, para cada $x \in G$, o subespaço vetorial de $T_{x} G$ dado por

$$
D_{x} \doteq \operatorname{span}\left\{\phi_{*} X(x) ; \phi \in \operatorname{Gr}(\Gamma), X \in \Gamma\right\}
$$


Lema 2.10. Para cada $x \in G$ temos que

$$
D_{x}=\operatorname{span}\left\{A d(y) X(x) ; y \in \mathcal{O}_{\Gamma}, X \in \Gamma\right\} .
$$

Demonstração. De acordo com o Corolário 2.8 todo $\phi \in \operatorname{Gr}(\Gamma)$ pode ser escrito como $\phi=R_{\phi(e)}$, de modo que, dado $X \in \Gamma$, temos que

$$
\phi_{*} X=\left(R_{\phi(e)}\right)_{*} X=\operatorname{Ad}\left(\phi^{-1}(e)\right) X .
$$

Como todo $y \in \mathcal{O}_{\Gamma}$ é da forma $y=\phi(e)$ para algum $\phi \in \operatorname{Gr}(\Gamma)$ e $\mathcal{O}_{\Gamma}$ é um grupo, concluímos que

$$
\left\{\phi_{*} X(x) ; \phi \in \operatorname{Gr}(\Gamma), X \in \Gamma\right\}=\left\{\operatorname{Ad}(y) X(x) ; y \in \mathcal{O}_{\Gamma}, X \in \Gamma\right\}
$$

donde segue nossa tese.

Lema 2.11. Se $x_{1}, x_{2} \in G$ então $\operatorname{dim} D_{x_{1}}=\operatorname{dim} D_{x_{2}}$.

Demonstração. Se definirmos $z \doteq x_{1} \cdot x_{2}^{-1}$ temos que $x_{1}=z \cdot x_{2}$. Assim, se $y \in \mathcal{O}_{\Gamma}$ e $X \in \Gamma$ temos que

$$
\begin{aligned}
\operatorname{Ad}(y) X\left(x_{1}\right) & =\operatorname{Ad}(y) X\left(z \cdot x_{2}\right) \\
& =\operatorname{Ad}(y) X\left(L_{z} x_{2}\right) \\
& =d L_{z} \operatorname{Ad}(y) X\left(x_{2}\right)
\end{aligned}
$$

pois $\operatorname{Ad}(y) X$ é invariante à esquerda. O argumento acima e o lema anterior implicam que

$$
D_{x_{1}}=d L_{z} D_{x_{2}}
$$

e como $d L_{z}$ é um isomorfismo linear concluímos que $D_{x_{1}}$ e $D_{x_{2}}$ têm a mesma dimensão.

Já estamos em condições de demonstrar o Teorema da Órbita para grupos de Lie.

Teorema 2.12 (da Órbita). Se $\Gamma \subset \mathfrak{g}$ então $H \doteq \mathcal{O}_{\Gamma}$ é um subgrupo de Lie conexo de $G$, cuja álgebra de Lie é

$$
\mathfrak{h}=\operatorname{Lie}(\Gamma)
$$

Demonstração. Já vimos que $\mathcal{O}_{\Gamma}$ possui uma estrutura de subvariedade imersa de $G$ fornecida pelo Teorema 1.3, a qual é conexa. Além disso, a Proposição 2.9 nos diz que $\mathcal{O}_{\Gamma}$ é um subgrupo de $G$ : para demonstrá-lo um subgrupo de Lie, resta mostrar que as operações de multiplicação e inversão

$$
\begin{aligned}
\mu: \mathcal{O}_{\Gamma} \times \mathcal{O}_{\Gamma} & \longrightarrow \mathcal{O}_{\Gamma} \\
(x, y) & \longmapsto x \cdot y
\end{aligned}
$$


$\mathrm{e}$

$$
\begin{aligned}
\lambda: \mathcal{O}_{\Gamma} & \longrightarrow \mathcal{O}_{\Gamma} \\
x & \longmapsto x^{-1}
\end{aligned}
$$

são suaves. Para isto, definimos

$$
D \doteq \bigcup_{x \in G}\{x\} \times D_{x} \subset T G
$$

onde $D_{x}$ é dado por (2.8). Como de acordo com o lema anterior a dimensão de $D_{x}$ é independente de $x$ temos que $D$ é uma distribuição no sentido usual, a qual é suave ${ }^{5}$. O Teorema 1.3 nos diz que fixado $x_{0} \in G$ temos, para cada $x \in \mathcal{O}_{\Gamma}\left(x_{0}\right)$, que

$$
T_{x} \mathcal{O}_{\Gamma}\left(x_{0}\right)=\operatorname{span}\left\{\phi_{*} X(x) ; \phi \in \operatorname{Gr}(\Gamma), X \in \Gamma\right\}=D_{x}
$$

ou seja, $\mathcal{O}_{\Gamma}\left(x_{0}\right)$ é uma variedade integral de $D$. Isto prova que $D$ é uma distribuição integrável e, portanto, involutiva ${ }^{6}$.

Agora, como $\mu$ e $\lambda$ são as restrições das operações em $G$ - que são suaves - e $\mathcal{O}_{\Gamma}$ é uma subvariedade imersa de $G$, não há nenhuma dificuldade com a restrição dos domínios. Ainda, vimos acima que $\mathcal{O}_{\Gamma}$ é uma variedade integral de uma distribuição involutiva, o que garante ${ }^{7}$ que também não teremos problema com a restrição dos contradomínios e prova que $\mu$ e $\lambda$ definidas acima são suaves. Isto prova que $\mathcal{O}_{\Gamma}$ é um subgrupo de Lie de $G$.

Quanto a sua álgebra de Lie, temos que $\Gamma \subset \mathfrak{g}$ implica que $\operatorname{Lie}(\Gamma) \subset \mathfrak{g}$ e, portanto, $\operatorname{Lie}(\Gamma)$ tem dimensão finita. Segue então da Proposição 1.6 que

$$
\operatorname{Lie}_{e}(\Gamma)=T_{e} \mathcal{O}_{\Gamma}(e)=T_{e} \mathcal{O}_{\Gamma}
$$

que é precisamente a álgebra de Lie de $\mathcal{O}_{\Gamma}$ (via identificação canônica). Desse modo

$$
\operatorname{Lie}(\Gamma)=\mathfrak{h}
$$

O teorema anterior nos fornece uma caracterização bastante conveniente da álgebra de Lie gerada por uma família invariante à esquerda.

Corolário 2.13. Se $\Gamma \subset \mathfrak{g}$ então

$$
\operatorname{Lie}(\Gamma)=\operatorname{span}\left\{A d(x) X ; x \in \mathcal{O}_{\Gamma}, X \in \Gamma\right\} .
$$

\footnotetext{
5 [Lee03], Lemma 19.1, página 495.

6 [Lee03], Proposition 19.3, página 497.

7 [Lee03], Proposition 19.13, página 505.
} 
Demonstração. Se denotarmos $H \doteq \mathcal{O}_{\Gamma}$ então, pelo teorema anterior,

$$
\mathfrak{h} \doteq \operatorname{Lie}(\Gamma)
$$

é a álgebra de Lie de $H$. Contudo

$$
\begin{aligned}
T_{e} H & =\operatorname{span}\left\{\phi_{*} X(e) ; \phi \in \operatorname{Gr}(\Gamma), X \in \Gamma\right\} \\
& =D_{e} \\
& =\operatorname{span}\left\{\operatorname{Ad}(x) X(e) ; x \in \mathcal{O}_{\Gamma}, X \in \Gamma\right\}
\end{aligned}
$$

conforme vimos acima. A tese segue da identificação canônica $\mathfrak{h} \cong T_{e} H$.

Proposição 2.14. Seja $\Gamma \subset \mathfrak{g}$. São equivalentes:

1. $\operatorname{Lie}(\Gamma)=\mathfrak{g}$;

2. $\Gamma$ é Lie-determinada i.e. Lie $_{x}(\Gamma)=T_{x} G$ para todo $x \in G$; e

3. $\operatorname{Lie}_{x}(\Gamma)=T_{x} G$ para algum $x \in G$.

Demonstração. É claro que $1 \Rightarrow 2 \Rightarrow 3$ : vamos mostrar que $3 \Rightarrow 1$.

Seja $x \in G$ tal que $\operatorname{Lie}_{x}(\Gamma)=T_{x} G$. É claro que Lie( $(\Gamma)$ é um subespaço vetorial de $\mathfrak{g}$, de modo que para mostrar a igualdade basta demonstrar que ambos são isomorfos como espaços vetoriais. De fato, denotando por ev $: \mathfrak{g} \rightarrow T_{e} G$ o isomorfismo canônico (avaliação na identidade) temos a seguinte sequência de isomorfismos lineares:

$$
\mathfrak{g} \stackrel{\mathrm{ev}}{\longrightarrow} T_{e} G \stackrel{d\left(L_{x}\right)_{e}}{\longrightarrow} T_{x} G=\operatorname{Lie}_{x}(\Gamma) \stackrel{d\left(L_{x}^{-1}\right)_{x}}{\longrightarrow} \operatorname{Lie}_{e}(\Gamma) \stackrel{\mathrm{ev}^{-1}}{\longrightarrow} \operatorname{Lie}(\Gamma)
$$

Desse modo, $\mathfrak{g}$ e Lie $(\Gamma)$ são espaços vetoriais isomorfos, o que conclui nossa prova.

\subsection{Particularidades dos grupos de Lie compactos}

Nesta seção denotaremos por $G$ um grupo de Lie compacto e conexo e por $\mathfrak{g}$ sua álgebra de Lie. A hipótese adicional de compacidade é fundamental para os resultados que enunciaremos nesta seção.

Dado um produto interno $\langle\cdot, \cdot\rangle$ em $\mathfrak{g}$, dizemos que ele é $A d$-invariante se para todo $x \in G$ vale que

$$
\langle\operatorname{Ad}(x) X, \operatorname{Ad}(x) Y\rangle=\langle X, Y\rangle
$$

para quaisquer $X, Y \in \mathfrak{g}$, isto é $\operatorname{Ad}(x)$ é uma isometria de $\mathfrak{g}$ com relação ao produto interno em questão. 
Proposição 2.15. Se $\langle\cdot, \cdot\rangle$ é um produto interno Ad-invariante em $\mathfrak{g}$ então para todo $X \in \mathfrak{g}$ vale que

$$
\langle Y, \operatorname{ad}(X) Z\rangle=-\langle\operatorname{ad}(X) Y, Z\rangle \text { para quaisquer } Y, Z \in \mathfrak{g} \text {. }
$$

Ademais - e mais importante - tais produtos internos existem.

Referência. [Kna02], Proposition 4.24, página 249.

Não é difícil ver que os produtos internos Ad-invariantes em $\mathfrak{g}$ estão em bijeção com as métricas riemannianas bi-invariantes em $G$ : a proposição acima nos diz, em particular, que todo grupo de Lie compacto admite uma métrica bi-invariante.

Uma consequência importante da proposição anterior é que a forma de Killing de uma álgebra de Lie oriunda de um grupo de Lie compacto é negativa semi-definida ${ }^{8}$. Outra consequência da proposição anterior - a qual utilizaremos no Corolário 4.9 - é que, no contexto de grupos de Lie compactos, $\operatorname{ad}(X)$ tem traço nulo para todo $X \in \mathfrak{g}$.

Corolário 2.16. Para cada $X \in \mathfrak{g}$ vale que

$$
\operatorname{tr} \operatorname{ad}(X)=0 \text {. }
$$

Demonstração. Sejam $\langle\cdot, \cdot\rangle$ um produto interno Ad-invariante em $\mathfrak{g}$ (cuja existência é garantida pela Proposição 2.15) e $Y_{1}, \ldots, Y_{n} \in \mathfrak{g}$ uma base ortonormal de $\mathfrak{g}$ com relação a esse produto interno. Então, aplicando mais uma vez a proposição anterior, temos

$$
\begin{aligned}
\operatorname{tr} \operatorname{ad}(X) & =\sum_{j=1}^{n}\left\langle\operatorname{ad}(X) Y_{j}, Y_{j}\right\rangle \\
& =-\sum_{j=1}^{n}\left\langle Y_{j}, \operatorname{ad}(X) Y_{j}\right\rangle \\
& =-\sum_{j=1}^{n}\left\langle\operatorname{ad}(X) Y_{j}, Y_{j}\right\rangle \\
& =-\operatorname{tr} \operatorname{ad}(X)
\end{aligned}
$$

o que implica que $\operatorname{tr} \operatorname{ad}(X)=0$.

Definimos o centro de $G$ como

$$
Z(G) \doteq\{x \in G ; x \cdot y=y \cdot x \text { para todo } y \in G\} .
$$

\footnotetext{
${ }^{8}$ [Kna02], Corollary 4.26, páginas 249 e 250.
} 
É possível mostrar que ${ }^{9}$

$$
Z(G)=\operatorname{ker} \mathrm{Ad}
$$

Como Ad : $G \rightarrow \mathrm{GL}(\mathfrak{g})$ é um homomorfismo de grupos de Lie segue que $Z(G)$ é um subgrupo de Lie fechado de $G$. Como ademais

$$
\mathfrak{z}(\mathfrak{g})=\operatorname{ker} \text { ad }
$$

temos que $\mathfrak{z}(\mathfrak{g})$ é a álgebra de Lie de $Z(G)$.

Sendo $G$ um grupo de Lie compacto, sua álgebra de Lie $\mathfrak{g}$ desfruta de uma propriedade especial: ela é redutiva ${ }^{10}$. Reunindo este fato às considerações feitas no final da Seção 2.1, deduzimos o seguinte teorema.

Teorema 2.17. Sejam $G$ um grupo de Lie compacto e $\mathfrak{g}$ sua álgebra de Lie. São equivalentes:

1. $\mathfrak{g}$ é semi-simples;

2. $\mathfrak{z}(\mathfrak{g})=\{0\}$

3. $Z(G)$ é um subgrupo discreto de $G$.

Se as condições equivalentes acima são satisfeitas, dizemos que $G$ é semi-simples . Já observamos que a forma de Killing $B$ em $\mathfrak{g}$ é uma forma bilinear simétrica, que é negativa semi-definida se $G$ for compacto. Se, ademais, $G$ for semi-simples então $B$ é não-degenerada e, portanto, negativa definida i.e. $-B$ é um produto interno em $\mathfrak{g}$.

\subsection{Comentários e referências}

Os resultados da Seção 2.1 foram todos extraídos de [Kna02], com mudanças mínimas de notação e eventual refraseamento dos teoremas. A única diferença fundamental é nossa definição de álgebra de Lie semi-simples, diferença esta que é inofensiva em razão do Critério de Cartan para semisimplicidade (veja [Kna02], Theorem 1.45, página 50). A Seção 2.2 também é mera seleção de tópicos da teoria dos grupos de Lie, sem nenhum acréscimo significativo em comparação às apresentações feitas usualmente em textos introdutórios da teoria de variedades suaves como, por exemplo, [Lee03].

Exceto por mudanças de notação e uma abordagem talvez um pouco mais abstrata, a Seção 2.3 foi essencialmente inspirada em [Sac09], que é uma excelente introdução à teoria de controle em grupos de Lie (veja também os Capítulos 2 e 6 de [Jur97]). Fizemos apenas questão de detalhar um argumento que justifica um ponto que julgamos obscuro em toda a literatura que encontramos a respeito, o da estrutura de grupo de Lie na órbita de uma família invariante à esquerda: para nós, está claro na literatura que este é um subgrupo abstrato que possui uma estrutura de subvariedade

\footnotetext{
9 [Sep07], Lemma 5.11, página 102.

10 [Kna02], Corollary 4.25, página 249.
} 
imersa, mas não que tais estruturas (a algébrica e a geométrica) são compatíveis no sentido de grupos de Lie i.e. que as operações de multiplicação e inversão são suaves. Nosso Lema 2.11 resolve este problema mostrando que a distribuição (generalizada) dada pelo Teorema da Órbita é, no contexto de grupos de Lie, uma distribuição no sentido clássico (tem posto constante), o que nos permite empregar os resultados mais conhecidos ligados à integrabilidade desse tipo de distribuição, como fizemos na demonstração do Teorema 2.12.

Os resultados enunciados na Seção 2.4 podem ser encontrados em [Kna02] e [Sep07]. 


\section{Capítulo 3}

\section{Planejamento periódico de trajetórias}

Neste capítulo enunciamos o problema do planejamento periódico de trajetórias para sistemas afins sem arrasto em grupos de Lie compactos (Seção 3.1), demonstramos que a solução deste problema se reduz a um problema de estabilização de outro sistema (Seção 3.2) e introduzimos as notações necessárias para desenvolver nossos métodos de solução nos capítulos subsequentes.

\subsection{Enunciado do problema}

Seja $G$ um grupo de Lie compacto e conexo, cuja álgebra de Lie denotaremos por $\mathfrak{g}$. Fixados campos invariantes à esquerda $X_{1}, \ldots, X_{m} \in \mathfrak{g}$ denotamos

$$
\Gamma \doteq \operatorname{span}\left\{X_{1}, \ldots, X_{m}\right\}
$$

Segundo o Teorema da Órbita para grupos de Lie (Teorema 2.12) $\mathcal{O}_{\Gamma}$ é um subgrupo de Lie conexo de $G$ cuja álgebra de Lie é exatamente

$$
\operatorname{Lie}(\Gamma)=\operatorname{span}\left\{\operatorname{Ad}(x) X ; x \in \mathcal{O}_{\Gamma}, X \in \Gamma\right\}
$$

Considere o sistema afim sem arrasto

$$
\mathbf{x}^{\prime}=\sum_{k=1}^{m} u_{k} X_{k}(\mathbf{x})
$$

onde $u_{1}, \ldots, u_{m} \in \mathbb{R}$. Uma trajetória do sistema (3.1) é uma $(m+1)$-upla $\left(\mathbf{x}, u_{1}, \ldots, u_{m}\right)$ tal que

- $u_{1}, \ldots, u_{m}: \mathbb{R} \rightarrow \mathbb{R}$ são funções suaves - as quais às vezes chamaremos de controles - e

- $\mathbf{x}: \mathbb{R} \rightarrow G$ é uma curva integral do campo vetorial dependente do tempo

$$
X(t, x) \doteq \sum_{k=1}^{m} u_{k}(t) X_{k}(x)
$$

com condição inicial $\mathbf{x}(0) \in \mathcal{O}_{\Gamma}$. 
Observe que o campo vetorial dependente do tempo (3.2) é tangente a $\mathcal{O}_{\Gamma}$ i.e. se $x \in \mathcal{O}_{\Gamma}$ então

$$
X(t, x) \in T_{x} \mathcal{O}_{\Gamma} \text { para todo } t \in \mathbb{R} .
$$

Isto implica, em particular, que se $\left(\mathbf{x}, u_{1}, \ldots, u_{m}\right)$ é uma trajetória de $(3.1)$ então $\mathbf{x}(t) \in \mathcal{O}_{\Gamma}$ para todo $t \in \mathbb{R}$.

Definição 3.1. Seja $T>0$. Uma trajetória de referência $T$-periódica para o sistema (3.1) é uma trajetória $\left(\mathbf{x}_{\mathbf{r}}, u_{1}^{r}, \ldots, u_{m}^{r}\right)$ do sistema (3.1) tal que

$$
\mathbf{x}_{\mathbf{r}}, u_{1}^{r}, \ldots, u_{m}^{r} \text { são } T \text {-periódicas. }
$$

Ao efetuar os cálculos na seções subsequentes denotaremos, por conveniência,

$$
X_{r} \doteq \sum_{j=1}^{m} u_{j}^{r} X_{j}
$$

quando $\left(\mathbf{x}_{\mathbf{r}}, u_{1}^{r}, \ldots, u_{m}^{r}\right)$ for uma trajetória de referência do sistema (3.1), o qual ora consideraremos um campo vetorial dependente do tempo - do qual $\mathbf{x}_{\mathbf{r}}$ é uma curva integral -, ora consideraremos uma aplicação suave $X_{r}: \mathbb{R} \rightarrow \mathfrak{g}$.

Definição 3.2. O problema do planejamento periódico de trajetórias para o sistema (3.1) é o seguinte: fixados uma condição inicial $x_{0} \in \mathcal{O}_{\Gamma}$ e uma trajetória de referência $T$-periódica para o sistema (3.1), a qual denotaremos por $\left(\mathbf{x}_{\mathbf{r}}, u_{1}^{r}, \ldots, u_{m}^{r}\right)$, encontrar outra trajetória $\left(\mathbf{x}, u_{1}, \ldots, u_{m}\right)$ de (3.1) tal que $\mathbf{x}(0)=x_{0}$ e

$$
\lim _{t \rightarrow \infty} \mathbf{x}(t) \cdot \mathbf{x}_{\mathbf{r}}(t)^{-1}=e
$$

Denominamos $\mathbf{x} \cdot \mathbf{x}_{\mathbf{r}}{ }^{-1}$ de erro de rastreamento entre as duas trajetórias.

Por simplicidade, suporemos nas próximas seções que $\Gamma$ é Lie-determinada, isto é $\operatorname{Lie}(\Gamma)=\mathfrak{g}$ : segundo o Teorema de Rashevsky-Chow (Teorema 1.8) isto ocorre se e somente se $\mathcal{O}_{\Gamma}=G$, visto que $G$ é conexo por hipótese.

\subsection{Um sistema modificado}

Nesta seção pretendemos, em linhas gerais, transformar o problema do planejamento periódico de trajetórias para o sistema (3.1) em um problema de estabilização de um outro sistema, associado a uma trajetória de referência $T$-periódica fixada, através de uma mudança de coordenadas que descrevemos em seguida. Para tanto, voltamos a denotar por $G$ um grupo de Lie compacto e conexo com álgebra de Lie $\mathfrak{g}$.

Suponhamos, por hora, que $\left(\mathbf{x}_{\mathbf{r}}, u_{1}^{r}, \ldots, u_{m}^{r}\right)$ é uma trajetória de referência $T$-periódica do sis- 
tema (3.1) fixada. Fixamos $x_{0} \in G$ e denotamos

$$
x_{\infty} \doteq \mathbf{x}_{\mathbf{r}}(0)
$$

Teorema 3.3. Suponha que $\left(\mathbf{z}, v_{1}, \ldots, v_{m}\right)$ é uma trajetória ${ }^{1}$ do sistema

$$
\mathbf{z}^{\prime}=\sum_{k=1}^{m} v_{k} A d\left(\mathbf{x}_{\mathbf{r}}(t)\right) X_{k}(\mathbf{z})
$$

tal que

1. $\mathbf{z}(0)=x_{0} \cdot x_{\infty}^{-1} e$

2. $\lim _{t \rightarrow \infty} \mathbf{z}(t)=e$.

Então se definirmos

$$
\begin{aligned}
\mathbf{x} & \doteq \mathbf{z} \cdot \mathbf{x}_{\mathbf{r}} \\
u_{k} & \doteq v_{k}+u_{k}^{r}, \quad 1 \leq k \leq m,
\end{aligned}
$$

temos que $\left(\mathbf{x}, u_{1}, \ldots, u_{m}\right)$ é uma trajetória do sistema (3.1) que satisfaz

1. $\mathbf{x}(0)=x_{0} e$

2. $\lim _{t \rightarrow \infty} \mathbf{x}(t) \cdot \mathbf{x}_{\mathbf{r}}(t)^{-1}=e$.

Ou seja, $\left(\mathbf{x}, u_{1}, \ldots, u_{m}\right)$ resolve o problema do planejamento periódico de trajetórias para o sistema (3.1).

É claro que o enunciado acima é obtido por "engenharia reversa": primeiro definimos z como o erro de rastreamento entre as duas trajetórias do sistema (3.1) para então derivar o sistema (3.5). Demonstrá-lo exige apenas a fórmula da derivada de um produto de curvas em um grupo de Lie, a qual enunciamos abaixo em forma de lema e cuja demonstração omitimos por ser elementar.

Lema 3.4. Sejam $G$ um grupo de Lie e $\mathbf{x}_{\mathbf{1}}, \mathbf{x}_{\mathbf{2}}: \mathbb{R} \rightarrow G$ curvas suaves. Então

$$
\left(\mathbf{x}_{1} \cdot \mathbf{x}_{2}\right)^{\prime}=d R_{\mathbf{x}_{2}} \mathbf{x}_{1}{ }^{\prime}+d L_{\mathbf{x}_{1}} \mathbf{x}_{\mathbf{2}}{ }^{\prime}
$$

Demonstração do Teorema 3.3. Definindo $\left(\mathbf{x}, u_{1}, \ldots, u_{m}\right)$ como em (3.6)-(3.7) temos, pelo lema anterior, que

$$
\begin{aligned}
\mathbf{x}^{\prime} & =\left(\mathbf{z} \cdot \mathbf{x}_{\mathbf{r}}\right)^{\prime} \\
& =d R_{\mathbf{x}_{\mathbf{r}}} \mathbf{z}^{\prime}+d L_{\mathbf{z}} \mathbf{x}_{\mathbf{r}}{ }^{\prime} \\
& =d R_{\mathbf{x}_{\mathbf{r}}} \sum_{k=1}^{m} v_{k} \operatorname{Ad}\left(\mathbf{x}_{\mathbf{r}}\right) X_{k}(\mathbf{z})+d L_{\mathbf{z}} \sum_{k=1}^{m} u_{k}^{r} X_{k}\left(\mathbf{x}_{\mathbf{r}}\right)
\end{aligned}
$$

\footnotetext{
${ }^{1} \mathrm{~A}$ definição de trajetória do sistema (3.5) é análoga àquela de trajetória do sistema (3.1).
} 
onde

$$
\begin{aligned}
d R_{\mathbf{x}_{\mathbf{r}}} \sum_{k=1}^{m} v_{k} \operatorname{Ad}\left(\mathbf{x}_{\mathbf{r}}\right) X_{k}(\mathbf{z}) & =d R_{\mathbf{x}_{\mathbf{r}}} \sum_{k=1}^{m} v_{k}\left(R_{\mathbf{x}_{\mathbf{r}}-1}\right)_{*} X_{k}(\mathbf{z}) \\
& =d R_{\mathbf{x}_{\mathbf{r}}} \sum_{k=1}^{m} v_{k} d R_{\mathbf{x}_{\mathbf{r}}-1} X_{k}\left(R_{\mathbf{x}_{\mathbf{r}}} \mathbf{z}\right) \\
& =d R_{\mathbf{x}_{\mathbf{r}}} d R_{\mathbf{x}_{\mathbf{r}}}-1 \sum_{k=1}^{m} v_{k} X_{k}\left(\mathbf{z} \cdot \mathbf{x}_{\mathbf{r}}\right) \\
& =\sum_{k=1}^{m} v_{k} X_{k}(\mathbf{x})
\end{aligned}
$$

e

$$
\begin{aligned}
d L_{\mathbf{z}} \sum_{k=1}^{m} u_{k}^{r} X_{k}\left(\mathbf{x}_{\mathbf{r}}\right) & =\sum_{k=1}^{m} u_{k}^{r} d L_{\mathbf{z}} X_{k}\left(\mathbf{x}_{\mathbf{r}}\right) \\
& =\sum_{k=1}^{m} u_{k}^{r} X_{k}\left(L_{\mathbf{z}} \mathbf{x}_{\mathbf{r}}\right) \\
& =\sum_{k=1}^{m} u_{k}^{r} X_{k}\left(\mathbf{z} \cdot \mathbf{x}_{\mathbf{r}}\right) \\
& =\sum_{k=1}^{m} u_{k}^{r} X_{k}(\mathbf{x})
\end{aligned}
$$

de modo que, retomando os cálculos anteriores, temos

$$
\begin{aligned}
\mathbf{x}^{\prime} & =d R_{\mathbf{x} \mathbf{r}} \sum_{k=1}^{m} v_{k} \operatorname{Ad}\left(\mathbf{x}_{\mathbf{r}}\right) X_{k}(\mathbf{z})+d L_{\mathbf{z}} \sum_{k=1}^{m} u_{k}^{r} X_{k}\left(\mathbf{x}_{\mathbf{r}}\right) \\
& =\sum_{k=1}^{m} v_{k} X_{k}(\mathbf{x})+\sum_{k=1}^{m} u_{k}^{r} X_{k}(\mathbf{x}) \\
& =\sum_{k=1}^{m}\left(v_{k}+u_{k}^{r}\right) X_{k}(\mathbf{x}) \\
& =\sum_{k=1}^{m} u_{k} X_{k}(\mathbf{x})
\end{aligned}
$$

o que prova que $\left(\mathbf{x}, u_{1}, \ldots, u_{m}\right)$ assim definida é, de fato, uma trajetória do sistema (3.1).

Temos ainda

$$
\mathbf{x}(0)=\mathbf{z}(0) \cdot \mathbf{x}_{\mathbf{r}}(0)=x_{0} \cdot x_{\infty}^{-1} \cdot x_{\infty}=x_{0}
$$


$\mathrm{e}$

$$
\lim _{t \rightarrow \infty} \mathbf{x}(t) \cdot \mathbf{x}_{\mathbf{r}}(t)^{-1}=\lim _{t \rightarrow \infty} \mathbf{z}(t)=e .
$$

Em vista do Teorema 3.3, a partir de agora nosso objetivo será encontrar uma trajetória $\left(\mathbf{z}, v_{1}, \ldots, v_{m}\right)$ do sistema $(3.5)$ - o qual está intimamente relacionado à trajetória de referência $\left(\mathbf{x}_{\mathbf{r}}, u_{1}^{r}, \ldots, u_{m}^{r}\right)-$ que satisfaça

1. $\mathbf{z}(0)=x_{0} \cdot x_{\infty}^{-1} \mathrm{e}$

2. $\lim _{t \rightarrow \infty} \mathbf{z}(t)=e$.

Com esta finalidade em mente, introduzimos a função "tipo Lyapunov" $V: G \rightarrow \mathbb{R}$ dada por

$$
V(x) \doteq \operatorname{tr} \operatorname{Ad}(x)
$$

e o campo auxiliar $W: \mathbb{R} \times G \rightarrow T G$ dado por

$$
W(t, w) \doteq \sum_{k=1}^{m} a_{k}(t, w) \operatorname{Ad}\left(\mathbf{x}_{\mathbf{r}}(t)\right) X_{k}(w)
$$

onde

$$
a_{k}(t, w) \doteq d V\left(\operatorname{Ad}\left(\mathbf{x}_{\mathbf{r}}(t)\right) X_{k}(w)\right), 1 \leq k \leq m
$$

Note que $W$ é um campo vetorial suave dependente do tempo que não é invariante à esquerda. Observe ainda que se $\mathbf{w}: \mathbb{R} \rightarrow G$ é uma curva integral de $W$ então se definirmos

$$
v_{k}(t) \doteq a_{k}(t, \mathbf{w}(t)), t \in \mathbb{R}
$$

temos que $\left(\mathbf{w}, v_{1}, \ldots, v_{m}\right)$ é trajetória do sistema (3.5).

Antes de prosseguirmos, vejamos porque a introdução do campo auxiliar nos ajudará a resolver o problema do planejamento periódico de trajetórias, e em qual sentido. Denotaremos por

$$
E_{W} \doteq\{w \in G ; W(t, w)=0 \text { para todo } t \in \mathbb{R}\}
$$

o conjunto dos pontos de equilíbrio de $W$.

Definição 3.5. Dizemos que $w \in E_{W}$ é um atrator local de $W$ se existe $U \subset G$ vizinhança de $w$ com a seguinte propriedade: se $\left(t_{0}, w_{0}\right) \in \mathbb{R} \times U$ e w $: \mathbb{R} \rightarrow G$ é a única curva integral de $W$ que satisfaz $\mathbf{w}\left(t_{0}\right)=w_{0}$ então

$$
\lim _{t \rightarrow \infty} \mathbf{w}(t)=w
$$


Proposição 3.6. Suponha que e $\in E_{W}$ e que é um atrator local de $W$. Então existe $U_{\infty} \subset G$ vizinhança de $x_{\infty}$ tal que se $x_{0} \in U_{\infty}$ então existe uma trajetória $\left(\mathbf{x}, u_{1}, \ldots, u_{m}\right)$ do sistema (3.1) que satisfaz

- $\mathbf{x}(0)=x_{0} e$

- $\lim _{t \rightarrow \infty} \mathbf{x}(t) \cdot \mathbf{x}_{\mathbf{r}}(t)^{-1}=e$

a qual é obtida da seguinte forma: tomamos $\mathbf{w}: \mathbb{R} \rightarrow G$ a única curva integral de $W$ que satisfaz $\mathbf{w}(0)=x_{0} \cdot x_{\infty}^{-1}$ e definimos

$$
\begin{aligned}
\mathbf{x}(t) & \doteq \mathbf{w}(t) \cdot \mathbf{x}_{\mathbf{r}}(t) \\
u_{k}(t) & \doteq a_{k}(t, \mathbf{w}(t))+u_{k}^{r}(t), 1 \leq k \leq m,
\end{aligned}
$$

para todo $t \in \mathbb{R}$.

Demonstração. Se $e \in E_{W}$ é um atrator local de $W$ então por definição existe $U \subset G$ vizinhança de $e$ tal que se $w_{0} \in U$ e $\mathbf{w}: \mathbb{R} \rightarrow G$ denota a única curva integral de $W$ que satisfaz $\mathbf{w}(0)=w_{0}$ então

$$
\lim _{t \rightarrow \infty} \mathbf{w}(t)=e .
$$

Definimos $U_{\infty} \doteq U \cdot x_{\infty}$, a qual é necessariamente uma vizinhança de $x_{\infty}$ (pois $e \in U$ ). Se $x_{0} \in U_{\infty}$ então $x_{0} \cdot x_{\infty}^{-1} \in U$ e logo se denotarmos por $\mathbf{w}: \mathbb{R} \rightarrow G$ a única curva integral de $W$ que satisfaz $\mathbf{w}(0)=x_{0} \cdot x_{\infty}^{-1}$ teremos

$$
\lim _{t \rightarrow \infty} \mathbf{w}(t)=e
$$

Já vimos, contudo, que se definirmos

$$
v_{k}(t) \doteq a_{k}(t, \mathbf{w}(t)), t \in \mathbb{R}
$$

para cada $k=1, \ldots, m$ teremos que $\left(\mathbf{w}, v_{1}, \ldots, v_{m}\right)$ é trajetória do sistema (3.5) que, em resumo, satisfaz

1. $\mathbf{w}(0)=x_{0} \cdot x_{\infty}^{-1} \mathrm{e}$

2. $\lim _{t \rightarrow \infty} \mathbf{w}(t)=e$.

Segue então do Teorema 3.3 que

$$
\begin{aligned}
\mathbf{x} & \doteq \mathbf{w} \cdot \mathbf{x}_{\mathbf{r}} \\
u_{k} & \doteq v_{k}+u_{k}^{r}, \quad 1 \leq k \leq m,
\end{aligned}
$$

define uma trajetória $\left(\mathbf{x}, u_{1}, \ldots, u_{m}\right)$ do sistema (3.1) que satisfaz 
1. $\mathbf{x}(0)=x_{0} \mathrm{e}$

2. $\lim _{t \rightarrow \infty} \mathbf{x}(t) \cdot \mathbf{x}_{\mathbf{r}}(t)^{-1}=e$

e resolve, portanto, o problema do planejamento periódico de trajetórias.

\subsection{Comentários e referências}

O problema do planejamento periódico de trajetórias para sistemas afins sem arrasto é tratado em [SdSR09] e no Capítulo 4 de [Sil09] no caso particular em que o espaço de estados é o grupo de Lie compacto $\mathrm{SU}(n)$. A técnica de introduzir a mudança de coordenadas (3.6)-(3.7) e uma função "tipo Lyapunov" usando o traço (3.8) para então reduzir o problema original ao estudo da estabilidade de um campo auxiliar (3.9) foi adaptada diretamente destas referências.

A ideia que motiva problema do planejamento periódico de trajetórias é o seguinte problema de controlabilidade: dados uma condição inicial $x_{0} \in G$ e um estado objetivo $x_{\infty} \in G$, encontrar uma trajetória $\left(\mathbf{x}, u_{1}, \ldots, u_{m}\right)$ de $(3.1)$ tal que, para algum $T>0$ fixado, temos

$$
\lim _{k \rightarrow \infty} \mathbf{x}(k T)=x_{\infty}
$$

É imediato verificar que se resolvermos o problema do planejamento periódico então também resolvemos o problema de controlabilidade definido acima para $x_{\infty} \doteq \mathbf{x}_{\mathbf{r}}(0)$.

A nomenclatura empregada na Definição 3.2 é motivada por exemplos concretos de grupos de Lie: se $G \subset \operatorname{GL}(n, \mathbb{C})$ é um subgrupo de Lie compacto e conexo e $\mathbf{x}, \mathbf{y}: \mathbb{R} \rightarrow G$ são curvas então

$$
\lim _{t \rightarrow \infty} \mathbf{x}(t) \cdot \mathbf{y}(t)^{-1}=e \Leftrightarrow \lim _{t \rightarrow \infty}\|\mathbf{x}(t)-\mathbf{y}(t)\|=0
$$

onde $\|\cdot\|$ denota qualquer norma definida em $\mathfrak{g l}(n, \mathbb{C})$. Uma prova bastante simples deste fato no caso particular $G=\mathrm{SU}(n)$ encontra-se em [Sil09], Proposição 4.1, página 84, e pode ser facilmente adaptada para o caso geral sugerido acima. Motivados por esta observação interpretamos, também no caso abstrato, a condição (3.4) como a curva $\mathbf{x}$ rastreia a curva $\mathbf{x}_{\mathbf{r}}$ fixada.

No caso particular em que $G=\mathrm{SU}(n)$ i.e.

$$
\begin{aligned}
G & =\left\{x \in \mathrm{GL}(n, \mathbb{C}) ; x^{*} x=e, \operatorname{det} x=1\right\} \\
\mathfrak{g} & =\left\{X \in \mathfrak{g l}(n, \mathbb{C}) ; X^{*}=-X, \operatorname{tr} X=0\right\}
\end{aligned}
$$

ou seja $\mathfrak{g}=\mathfrak{s u}(n)$, onde $e \doteq \operatorname{id}_{\mathbb{C}^{n}}$ e $x^{*}$ denota a transposta conjugada de $x$ : sendo $G$ um grupo de Lie linear i.e. um subgrupo fechado de $\operatorname{GL}(n, \mathbb{C})$ temos que ${ }^{2}$

$$
\begin{aligned}
\operatorname{Ad}(x) X & =x \cdot X \cdot x^{-1} \\
& =x \cdot X \cdot x^{*}
\end{aligned}
$$

\footnotetext{
${ }^{2}$ [Kna02], página 79.
} 
para quaisquer $x \in G$ e $X \in \mathfrak{g}$, onde na última igualdade usamos o fato de que $x^{-1}=x^{*}$ para todo $x \in \mathrm{SU}(n)$. Assim obtemos a seguinte expressão para o sistema (3.5)

$$
\begin{aligned}
\mathbf{z}^{\prime} & =\sum_{k=1}^{m} v_{k} \operatorname{Ad}\left(\mathbf{x}_{\mathbf{r}}\right) X_{k}(\mathbf{z}) \\
& =\sum_{k=1}^{m} v_{k} \operatorname{Ad}\left(\mathbf{x}_{\mathbf{r}}\right) X_{k}(\mathbf{z} \cdot e) \\
& =\sum_{k=1}^{m} v_{k} d L_{\mathbf{z}} \operatorname{Ad}\left(\mathbf{x}_{\mathbf{r}}\right) X_{k}(e) \\
& =\sum_{k=1}^{m} v_{k} d L_{\mathbf{z}} \operatorname{Ad}\left(\mathbf{x}_{\mathbf{r}}\right) X_{k}(e) \\
& =\sum_{k=1}^{m} v_{k} \mathbf{z} \cdot \mathbf{x}_{\mathbf{r}} \cdot X_{k} \cdot \mathbf{x}_{\mathbf{r}}{ }^{*} \\
& =\mathbf{z} \cdot \mathbf{x}_{\mathbf{r}} \cdot \sum_{k=1}^{m} v_{k} X_{k} \cdot \mathbf{x}_{\mathbf{r}}{ }^{*}
\end{aligned}
$$

e analogamente para o campo auxiliar (3.9)

$$
W(t, w)=w \cdot \mathbf{x}_{\mathbf{r}}(t) \cdot \sum_{k=1}^{m} a_{k}(t, w) X_{k} \cdot \mathbf{x}_{\mathbf{r}}(t)^{*}
$$

onde

$$
\begin{aligned}
a_{k}(t, w) & =d V\left(\operatorname{Ad}\left(\mathbf{x}_{\mathbf{r}}(t)\right) X_{k}(w)\right) \\
& =d V\left(w \cdot \mathbf{x}_{\mathbf{r}}(t) \cdot X_{k} \cdot \mathbf{x}_{\mathbf{r}}(t)^{*}\right)
\end{aligned}
$$

para $k=1, \ldots, m$. Sugerimos ao leitor que compare as expressões acima com aquelas apresentadas em [Sil09], páginas 82-84 (sistema (3.5)) e página 89 (campo auxiliar): a principal diferença é com relação à mudança de coordenadas, que definimos de forma um pouco diferente da versão original (veja [Sil09], página 82) visto que nossos campos são por definição invariantes à esquerda, e não à direita, modificação esta que é irrelevante do ponto de vista qualitativo e afeta minimamente o formato das equações obtidas.

Ao tentar reproduzir a técnica descrita acima em um contexto mais abstrato, porém, observamos que a representação adjunta do grupo de Lie em questão aparece naturalmente ao aplicar a tal mudança de coordenadas (Teorema 3.3), tornando a expressão do sistema (3.5) muito mais elegante. Mais que isso, agora nossa função "tipo Lyapunov" (3.8) é simplesmente o caractere da representação adjunta: essa informação nos dá uma orientação muito forte do tipo de teoria que teremos de empregar para estudar o problema em questão.

Observe que o Teorema 3.3 e a Proposição 3.6 não somente resolvem o problema do planejamento periódico de trajetórias em uma vizinhança do estado objetivo $x_{\infty}$, mas dão uma "receita" para 
encontrar os controles da trajetória que rastreará a trajetória de referência: consiste em integrar o campo auxiliar $W$ com condições iniciais em uma vizinhança atratora da identidade.

Em suma, se provarmos que

- $e \in E_{W} \mathrm{e}$

- $e$ é um atrator local de $W$

então o problema do planejamento periódico de trajetórias estará resolvido em uma vizinhança do estado objetivo $x_{\infty}$. Nosso objetivo, daqui para frente, será provar as duas afirmações listadas acima.

A primeira afirmação não requer hipóteses adicionais, e será demonstrada logo no início da Seção 4.2 (Corolário 4.9). A segunda afirmação é bastante mais delicada e dependerá fortemente da natureza do espaço de estados: se $e$ for um atrator local de $W$ então necessariamente $G$ é um grupo de Lie semi-simples (Corolário 4.9). Mesmo com essa hipótese, demonstraremos a segunda afirmação apenas para uma classe de trajetórias de referência, as ditas regulares, com uma ressalva: só provamos a existência de trajetórias regulares para uma classe particular de sistemas afins. Para chegar a demonstrar estes resultados, teremos que entender mais profundamente o comportamento assintótico das curvas integrais do campo auxiliar, o que faremos no Capítulo 4.

Apesar de estarmos supondo que $\Gamma$ é Lie-determinada, acreditamos fortemente que essa hipótese pode ser eliminada com pequenas adaptações nos resultados aqui apresentados: a ideia, no caso $\operatorname{Lie}(\Gamma) \neq \mathfrak{g}$, seria restringir o problema à órbita $\mathcal{O}_{\Gamma}$, onde $\Gamma$ é uma família Lie-determinada e, portanto, espera-se que nossos resultados valham em algum sentido. É claro, contudo, que neste caso as questões de convergência devem ser interpretadas também no contexto da órbita (não esperamos, por exemplo, que a identidade possua uma vizinhança atratora em $G$, mas sim que possua uma tal vizinhança em $\mathcal{O}_{\Gamma}$ ). Infelizmente não houve tempo para analisarmos este caso no presente trabalho, no qual restringimo-nos apenas ao caso Lie-determinado. Tampouco estudamos o algoritmo proposto por [Sil09], Algoritmo 4.22 e Teorema 4.23, páginas 111-114, para detectar uma vizinhança atratora da identidade e obter um resultado global.

Por fim, vale ressaltar que, em termos práticos, a introdução do campo auxiliar é desnecessária, no sentido de que poderíamos enunciar e deduzir todos os resultados deste trabalho apenas em termos do sistema (3.5) e resolver o problema do planejamento periódico da mesma forma: o papel do campo auxiliar é puramente didático, pois codifica em uma única estrutura o sistema (3.5) e a escolha de controles (3.10), simplificando a notação e os enunciados dos teoremas. 


\section{Capítulo 4}

\section{Estabilidade}

Este capítulo tem dois objetivos. Na Seção 4.1 discutimos alguns resultados a respeito de estabilidade de campos vetoriais dependentes do tempo em variedades compactas. Em seguida (Seção 4.2), empregamos estes resultados para demonstrar várias propriedades importantes dos conjuntos $\Omega$-limite (Definição 4.1) do campo auxiliar $W$ (3.9), culminando na demonstração do Teorema 4.7, que essencialmente diz que todo ponto $\Omega$-limite de $W$ é um ponto de equilíbrio.

\subsection{Alguns resultados de estabilidade}

A fim de compreender melhor o comportamento assintótico das curvas integrais do campo auxiliar (3.9), dedicamos esta seção à introdução de alguns conceitos e resultados ligados à estabilidade de campos vetoriais dependentes do tempo em variedades compactas.

Sejam $M$ uma variedade - a qual, a título de simplicidade, suporemos compacta até o fim desta seção - e $X: \mathbb{R} \times M \rightarrow T M$ um campo vetorial suave dependente do tempo. A hipótese de compacidade de $M$ implica que as curvas integrais de $X$ estão definidas em $\mathbb{R}$.

Definição 4.1. Sejam $\left(t_{0}, x_{0}\right) \in \mathbb{R} \times M$ e $\mathbf{x}: \mathbb{R} \rightarrow M$ a única curva integral de $X$ que satisfaz $\mathbf{x}\left(t_{0}\right)=x_{0}$. Dizemos que $x \in M$ é um ponto $\Omega$-limite de $\left(t_{0}, x_{0}\right)$ com relação ao campo $X$ se existe uma sequência $\left(t_{n}\right)_{n \in \mathbb{N}}$ em $\mathbb{R}$, crescente e ilimitada superiormente, tal que

$$
\lim _{n \rightarrow \infty} \mathbf{x}\left(t_{n}\right)=x
$$

O conjunto dos pontos $\Omega$-limite de $\left(t_{0}, x_{0}\right)$ é chamado conjunto $\Omega$-limite e é denotado por $\Omega_{X}\left(t_{0}, x_{0}\right)$.

Um resultado bem conhecido - e de fácil demonstração - nos diz que a compacidade de $M$ implica que $\Omega_{X}\left(t_{0}, x_{0}\right) \neq \emptyset$ para todo par $\left(t_{0}, x_{0}\right) \in \mathbb{R} \times M$ (qualquer que seja o campo $X$ ).

Definição 4.2. Dizemos que uma função contínua $V: M \rightarrow \mathbb{R}$ é não decrescente ao longo de $X$ se para toda curva integral $\mathbf{x}: \mathbb{R} \rightarrow M$ de $X$ temos que $V \circ \mathbf{x}: \mathbb{R} \rightarrow \mathbb{R}$ é uma função não decrescente.

Por exemplo, se $V \in C^{\infty}(M)$ satisfaz

$$
d V(X(t, x)) \geq 0 \text { para todo par }(t, x) \in \mathbb{R} \times M
$$


então $V$ é não decrescente ao longo de $X$.

Proposição 4.3. Sejam $V: M \rightarrow \mathbb{R}$ contínua e não decrescente ao longo de $X$ e $\left(t_{0}, x_{0}\right) \in \mathbb{R} \times M$ fixado. Então $V$ é constante em $\Omega_{X}\left(t_{0}, x_{0}\right)$ : se $x_{1}, x_{2} \in \Omega_{X}\left(t_{0}, x_{0}\right)$ então

$$
V\left(x_{1}\right)=V\left(x_{2}\right)
$$

Demonstração. Seja $\mathbf{x}: \mathbb{R} \rightarrow M$ a única curva integral de $X$ que satisfaz $\mathbf{x}\left(t_{0}\right)=x_{0}$. Para $j=1,2$, $x_{j} \in \Omega_{X}\left(t_{0}, x_{0}\right)$ implica que existe uma sequência crescente e ilimitada superiormente $\left(t_{n}^{j}\right)_{n \in \mathbb{N}}$ em $\mathbb{R}$ tal que

$$
\lim _{n \rightarrow \infty} \mathbf{x}\left(t_{n}^{j}\right)=x_{j}
$$

de modo que, sendo $V$ contínua, temos

$$
\lim _{n \rightarrow \infty} V\left(\mathbf{x}\left(t_{n}^{j}\right)\right)=V\left(x_{j}\right)
$$

Assim, como $V \circ \mathbf{x}$ é não decrescente por hipótese temos que

$$
V\left(\mathbf{x}\left(t_{n}^{j}\right)\right) \leq V\left(x_{j}\right) \text { para todo } n \in \mathbb{N}
$$

para $j=1,2$.

Extraímos então uma subsequência $\left(t_{n_{k}}^{2}\right)_{k \in \mathbb{N}}$ de $\left(t_{n}^{2}\right)_{n \in \mathbb{N}}$ com a propriedade de que

$$
t_{k}^{1} \leq t_{n_{k}}^{2} \text { para todo } k \in \mathbb{N}
$$

a qual pode ser obtida por indução em $k$. Como $V \circ \mathbf{x}$ é não decrescente temos que

$$
V\left(\mathbf{x}\left(t_{k}^{1}\right)\right) \leq V\left(\mathbf{x}\left(t_{n_{k}}^{2}\right)\right) \text { para todo } k \in \mathbb{N} \text {. }
$$

Contudo, já vimos que $V\left(\mathbf{x}\left(t_{n_{k}}^{2}\right)\right) \leq V\left(x_{2}\right)$ para todo $k \in \mathbb{N}$, de modo que

$$
V\left(\mathbf{x}\left(t_{k}^{1}\right)\right) \leq V\left(x_{2}\right) \text { para todo } k \in \mathbb{N}
$$

e, como $\lim _{k \rightarrow \infty} V\left(\mathbf{x}\left(t_{k}^{1}\right)\right)=V\left(x_{1}\right)$, concluímos que

$$
V\left(x_{1}\right) \leq V\left(x_{2}\right)
$$

Invertendo os papéis de $x_{1}$ e $x_{2}$ no argumento acima, obtemos a desigualdade recíproca e, portanto, a igualdade. 
Corolário 4.4. Se $V: M \rightarrow \mathbb{R}$ é contínua e não decrescente ao longo de $X$ então para qualquer $x \in \Omega_{X}\left(t_{0}, x_{0}\right)$ temos que

$$
\lim _{t \rightarrow \infty} V(\mathbf{x}(t))=V(x)
$$

onde $\mathbf{x}: \mathbb{R} \rightarrow M$ é a única curva integral de $X$ que satisfaz $\mathbf{x}\left(t_{0}\right)=x_{0}$.

Demonstração. É suficiente mostrar que toda sequência crescente e ilimitada superiormente $\left(t_{n}\right)_{n \in \mathbb{N}}$ em $\mathbb{R}$ possui uma subsequência $\left(t_{n_{k}}\right)_{k \in \mathbb{N}}$ com a propriedade de que

$$
\lim _{k \rightarrow \infty} V\left(\mathbf{x}\left(t_{n_{k}}\right)\right)=V(x)
$$

(É exatamente a contrapositiva da definição de limite: se supusermos a negativa de (4.1), encontramos uma sequência do tipo supracitado que não satisfaz a propriedade enunciada acima.)

Com efeito, se $\left(t_{n}\right)_{n \in \mathbb{N}}$ é uma tal sequência observe que, sendo $M$ compacta por hipótese, é necessário que exista uma subsequência de $\left(\mathbf{x}\left(t_{n}\right)\right)_{n \in \mathbb{N}}$ que convirja em $M$, digamos, para $\tilde{x} \in M$. Note que necessariamente $\tilde{x} \in \Omega_{X}\left(t_{0}, x_{0}\right)$ por definição de ponto $\Omega$-limite. Denotando por $\left(t_{n_{k}}\right)_{k \in \mathbb{N}}$ a subsequência de $\left(t_{n}\right)_{n \in \mathbb{N}}$ associada à tal subsequência i.e. tal que

$$
\lim _{k \rightarrow \infty} \mathbf{x}\left(t_{n_{k}}\right)=\tilde{x}
$$

e, da continuidade de $V$,

$$
\lim _{k \rightarrow \infty} V\left(\mathbf{x}\left(t_{n_{k}}\right)\right)=V(\tilde{x})
$$

No entanto, da proposição anterior temos que $V(\tilde{x})=V(x)$ pois $x, \tilde{x} \in \Omega_{X}\left(t_{0}, x_{0}\right)$. Concluímos que

$$
\lim _{k \rightarrow \infty} V\left(\mathbf{x}\left(t_{n_{k}}\right)\right)=V(x)
$$

o que encerra nossa demonstração pelo argumento anterior.

Os dois próximos resultados são totalmente independentes dos anteriores e não dependem da compacidade de $M$. Sua formulação, porém, requer a introdução de uma métrica riemanniana.

Lema 4.5. Suponha que $M$ é uma variedade riemanniana e seja $\mathbf{x}: \mathbb{R} \rightarrow M$ uma curva suave tal que

$$
\lim _{t \rightarrow \infty}\left\|\mathbf{x}^{\prime}(t)\right\|=0
$$

Suponha ainda que $x \in M$ é tal que existe uma sequência $\left(t_{n}\right)_{n \in \mathbb{N}}$ em $\mathbb{R}$, crescente e ilimitada superiormente, tal que

$$
\lim _{n \rightarrow \infty} \mathbf{x}\left(t_{n}\right)=x
$$


Então para todo $\epsilon \in \mathbb{R}$ temos que

$$
\lim _{n \rightarrow \infty} \mathbf{x}\left(t_{n}+\epsilon\right)=x
$$

Demonstração. Suponhamos sem perda de generalidade que $M$ é conexa: a continuidade de $\mathbf{x}$ implica que sua imagem está integralmente contida em uma componente conexa de $M$, a qual também contém necessariamente o ponto $x$ em vista das hipóteses acima.

Considere $d: M \times M \rightarrow \mathbb{R}$ a distância em $M$ induzida pela métrica riemanniana ${ }^{1}$. É um fato bem conhecido que a topologia induzida por $d$ coincide com a topologia original de $M^{2}$. Tendo esta observação em vista vamos mostrar, equivalentemente, que

$$
\lim _{n \rightarrow \infty} d\left(\mathbf{x}\left(t_{n}+\epsilon\right), x\right)=0
$$

qualquer que seja $\epsilon \in \mathbb{R}$.

Observe que para cada $n \in \mathbb{N}$ temos

$$
d\left(\mathbf{x}\left(t_{n}+\epsilon\right), x\right) \leq d\left(\mathbf{x}\left(t_{n}+\epsilon\right), \mathbf{x}\left(t_{n}\right)\right)+d\left(\mathbf{x}\left(t_{n}\right), x\right) .
$$

Como por hipótese temos

$$
\lim _{n \rightarrow \infty} d\left(\mathbf{x}\left(t_{n}\right), x\right)=0
$$

(pois $\lim _{n \rightarrow \infty} \mathbf{x}\left(t_{n}\right)=x$ ) é suficiente mostrar que

$$
\lim _{n \rightarrow \infty} d\left(\mathbf{x}\left(t_{n}+\epsilon\right), \mathbf{x}\left(t_{n}\right)\right)=0
$$

Para cada $n \in \mathbb{N}$ vale

$$
\begin{aligned}
d\left(\mathbf{x}\left(t_{n}+\epsilon\right), \mathbf{x}\left(t_{n}\right)\right) & \leq\left|\int_{t_{n}}^{t_{n}+\epsilon}\left\|\mathbf{x}^{\prime}(t)\right\| d t\right| \\
& \leq\left(\sup _{t \in\left[t_{n}, t_{n}+\epsilon\right]}\left\|\mathbf{x}^{\prime}(t)\right\|\right)|\epsilon|
\end{aligned}
$$

onde na primeira desigualdade usamos exatamente a definição de $d$, observando que $\left.\mathbf{x}\right|_{\left[t_{n}, t_{n}+\epsilon\right]}$ é um segmento de curva suave que conecta $\mathbf{x}\left(t_{n}\right)$ e $\mathbf{x}\left(t_{n}+\epsilon\right)^{3}$.

Note contudo que

$$
\lim _{n \rightarrow \infty} \sup _{t \in\left[t_{n}, t_{n}+\epsilon\right]}\left\|\mathbf{x}^{\prime}(t)\right\|=0 .
$$

\footnotetext{
${ }^{1} \mathrm{~A}$ distância entre dois pontos de $M$ é definida como o ínfimo dos comprimentos de todos os segmentos de curva suaves por partes que conectam os pontos em questão. Esta noção está bem definida em vista da hipótese de conexidade: veja [Lee03], Lemma 6.17, páginas 139 e 140.

2 [Lee03], Proposition 11.20, páginas 278 e 279.

${ }^{3}$ Aqui, estamos denotando por $\left[t_{n}, t_{n}+\epsilon\right]$ o intervalo fechado delimitado por $t_{n}$ e $t_{n}+\epsilon$, mesmo quando $\epsilon<0$.
} 
Com efeito, dado $\delta>0$ temos, por hipótese de que $\lim _{t \rightarrow \infty}\left\|\mathbf{x}^{\prime}(t)\right\|=0$, que existe $R>0$ tal que

$$
t>R \Rightarrow\left\|\mathbf{x}^{\prime}(t)\right\|<\delta
$$

Logo, como $\left(t_{n}\right)_{n \in \mathbb{N}}$ é crescente e ilimitada superiormente existe $n_{0} \in \mathbb{N}$ tal que

$$
\begin{aligned}
n \geq n_{0} & \Rightarrow \max \left\{t_{n}, t_{n}+\epsilon\right\}>R \\
& \Rightarrow \sup _{t \in\left[t_{n}, t_{n}+\epsilon\right]}\left\|\mathbf{x}^{\prime}(t)\right\|<\delta .
\end{aligned}
$$

Concluímos então da desigualdade anterior que

$$
\lim _{n \rightarrow \infty} d\left(\mathbf{x}\left(t_{n}+\epsilon\right), \mathbf{x}\left(t_{n}\right)\right)=0
$$

conforme prometido. Isto encerra a demonstração.

Se $A, B$ são conjuntos quaisquer, dizemos que uma função $f: \mathbb{R} \times A \rightarrow B$ é T-periódica se

$$
f(t+T, x)=f(t, x)
$$

para todo par $(t, x) \in \mathbb{R} \times A$.

Proposição 4.6. Sejam $M$ uma variedade riemanniana, $\mathbf{x}: \mathbb{R} \rightarrow M$ uma curva suave e $f$ : $\mathbb{R} \times M \rightarrow \mathbb{R}$ contínua tais que

- $\lim _{t \rightarrow \infty}\left\|\mathbf{x}^{\prime}(t)\right\|=0$,

- $f$ éT-periódica e

- $\lim _{t \rightarrow \infty} f(t, \mathbf{x}(t))=0$

Suponha ainda que $x \in M$ é tal que existe uma sequência $\left(t_{n}\right)_{n \in \mathbb{N}}$ em $\mathbb{R}$, crescente e ilimitada superiormente, tal que

$$
\lim _{n \rightarrow \infty} \mathbf{x}\left(t_{n}\right)=x
$$

Então

$$
f(s, x)=0 \text { para todo } s \in \mathbb{R} .
$$

Demonstração. Fixemos $s \in \mathbb{R}$ arbitrariamente. Para cada $n \in \mathbb{N}$ existe $l_{n} \in \mathbb{Z}$ tal que

$$
s_{n} \doteq t_{n}-l_{n} T
$$


é um elemento de $[0, T)$, de modo que a sequência $\left(s_{n}\right)_{n \in \mathbb{N}}$ possui uma subsequência convergente, digamos

$$
\lim _{k \rightarrow \infty} s_{n_{k}}=\theta \in[0, T]
$$

Definimos as novas sequências

$$
\begin{aligned}
s_{n_{k}}^{*} & \doteq s_{n_{k}}-\theta+s \\
t_{n_{k}}^{*} & \doteq t_{n_{k}}-\theta+s \\
& =s_{n_{k}}+l_{n_{k}} T-\theta+s \\
& =s_{n_{k}}^{*}+l_{n_{k}} T .
\end{aligned}
$$

para todo $k \in \mathbb{N}$. Observe que

$$
\lim _{k \rightarrow \infty} s_{n_{k}}=\theta \Rightarrow \lim _{k \rightarrow \infty} s_{n_{k}}^{*}=s .
$$

Recordando as hipóteses a respeito de $\mathbf{x}$ temos, segundo o Lema 4.5, que

$$
\lim _{k \rightarrow \infty} \mathbf{x}\left(t_{n_{k}}+\epsilon\right)=\lim _{k \rightarrow \infty} \mathbf{x}\left(t_{n_{k}}\right)=x
$$

para qualquer $\epsilon \in \mathbb{R}$. Tomando em particular $\epsilon \doteq-\theta+s$ temos

$$
\lim _{k \rightarrow \infty} \mathbf{x}\left(t_{n_{k}}^{*}\right)=\lim _{k \rightarrow \infty} \mathbf{x}\left(t_{n_{k}}-\theta+s\right)=x .
$$

Temos então da continuidade de $f$ que

$$
\begin{aligned}
f(s, x) & =\lim _{k \rightarrow \infty} f\left(s_{n_{k}}^{*}, \mathbf{x}\left(t_{n_{k}}^{*}\right)\right) \\
& =\lim _{k \rightarrow \infty} f\left(t_{n_{k}}^{*}-l_{n_{k}} T, \mathbf{x}\left(t_{n_{k}}^{*}\right)\right) \\
& =\lim _{k \rightarrow \infty} f\left(t_{n_{k}}^{*}, \mathbf{x}\left(t_{n_{k}}^{*}\right)\right) \\
& =0
\end{aligned}
$$

onde na terceira igualdade usamos que $f$ é $T$-periódica e na quarta igualdade usamos que

$$
\begin{aligned}
\lim _{t \rightarrow \infty} f(t, \mathbf{x}(t)) & =0 \\
\lim _{k \rightarrow \infty} t_{n_{k}}^{*} & =\infty .
\end{aligned}
$$




\subsection{Estudo dos conjuntos $\Omega$-limite - parte I}

Recapitulando a Seção 3.2, denotamos por $G$ um grupo de Lie compacto e conexo, com álgebra de Lie $\mathfrak{g}$, e por $W$ o campo auxiliar (3.9) associado a uma trajetória de referência $T$-periódica $\left(\mathbf{x}_{\mathbf{r}}, u_{1}^{r}, \ldots, u_{m}^{r}\right)$ fixada do sistema (3.1).

O objetivo desta seção é provar o

Teorema 4.7. Seja $\left(t_{0}, w_{0}\right) \in \mathbb{R} \times G$ fixado. Se $w \in \Omega_{W}\left(t_{0}, w_{0}\right)$ então

$$
a_{k}(t, w)=0 \text { para todo } t \in \mathbb{R}
$$

para todo $k=1, \ldots, m$.

Em particular, todo $w \in \Omega_{W}\left(t_{0}, w_{0}\right)$ é ponto de equilíbrio de $W$.

O teorema acima será um passo fundamental na caracterização dos conjuntos $\Omega$-limite do campo auxiliar $W$, como ficará mais claro ao longo desta e das próximas seções. Sua demonstração é um tanto técnica e depende de vários resultados menores, os quais são interessantes por si próprios e são enunciados e demonstrados a seguir.

Primeiramente, precisamos computar de maneira mais explícita os coeficientes $a_{k}$, tarefa esta que está intimamente ligada à compreensão do diferencial da função $V$.

Proposição 4.8. Para todo $(x, v) \in T G$ temos

$$
d V_{x} v=\operatorname{tr}\left\{A d(x) \cdot \operatorname{ad}\left(d L_{x^{-1}} v\right)\right\}
$$

Em particular, para cada $k=1, \ldots, m$ temos que

$$
a_{k}(t, w)=\operatorname{tr}\left\{A d(w) \cdot a d\left(A d\left(\mathbf{x}_{\mathbf{r}}(t)\right) X_{k}\right)\right\}
$$

para todo $(t, w) \in \mathbb{R} \times G$.

Demonstração. Primeiramente, observamos mais uma vez que se $(x, v) \in T G$ então $d L_{x^{-1}} v \in T_{e} G$ e, logo, o lado direito da equação (4.2) está bem definido em vista da identificação (2.4), conforme já discutido na demonstração da identidade (2.5).

Recordando agora que o traço $\operatorname{tr}: \mathfrak{g l}(\mathfrak{g}) \rightarrow \mathbb{R}$ é uma transformação linear e que por definição $V=\operatorname{tr} \circ \mathrm{Ad}$, a identidade acima segue imediatamente de (2.5) e da regra da cadeia.

Da definição de $a_{k}$ e da equação (4.2) temos, omitindo $t$ por simplicidade,

$$
\begin{aligned}
a_{k}(t, w) & =d V\left(\operatorname{Ad}\left(\mathbf{x}_{\mathbf{r}}\right) X_{k}(w)\right) \\
& =\operatorname{tr}\left\{\operatorname{Ad}(w) \cdot \operatorname{ad}\left(d L_{w^{-1}} \operatorname{Ad}\left(\mathbf{x}_{\mathbf{r}}\right) X_{k}(w)\right)\right\} \\
& =\operatorname{tr}\left\{\operatorname{Ad}(w) \cdot \operatorname{ad}\left(\operatorname{Ad}\left(\mathbf{x}_{\mathbf{r}}\right) X_{k}(e)\right)\right\} \\
& =\operatorname{tr}\left\{\operatorname{Ad}(w) \cdot \operatorname{ad}\left(\operatorname{Ad}\left(\mathbf{x}_{\mathbf{r}}\right) X_{k}\right)\right\}
\end{aligned}
$$

onde usamos que $\operatorname{Ad}\left(\mathbf{x}_{\mathbf{r}}(t)\right) X_{k}$ é invariante à esquerda para todo $t \in \mathbb{R}$. 
Já podemos esclarecer dois pontos a respeito de nossos objetivos que ficaram pendentes no fim do Capítulo 3.

\section{Corolário 4.9.}

1. Todo $w \in Z(G)$ é um ponto de equilíbrio do campo auxiliar $W$. Em particular, a identidade $e \in G$ é um ponto de equilíbrio de $W$.

2. Se e é um atrator local de $W$ então $G$ é semi-simples.

Demonstração. Recordando que $Z(G)=$ ker Ad (identidade (2.9)) temos que

$$
\operatorname{Ad}(w)=\operatorname{id}_{\mathfrak{g}} .
$$

Logo, de acordo com a proposição anterior temos, para cada $k=1, \ldots, m$,

$$
\begin{aligned}
a_{k}(t, w) & =\operatorname{tr}\left\{\operatorname{Ad}(w) \cdot \operatorname{ad}\left(\operatorname{Ad}\left(\mathbf{x}_{\mathbf{r}}\right) X_{k}\right)\right\} \\
& =\operatorname{tr}\left\{\operatorname{id}_{\mathfrak{g}} \cdot \operatorname{ad}\left(\operatorname{Ad}\left(\mathbf{x}_{\mathbf{r}}\right) X_{k}\right)\right\} \\
& =\operatorname{tr}\left\{\operatorname{ad}\left(\operatorname{Ad}\left(\mathbf{x}_{\mathbf{r}}\right) X_{k}\right)\right\} \\
& =0
\end{aligned}
$$

para todo $t \in \mathbb{R}$ já que, segundo o Corolário $2.16, \operatorname{ad}(X)$ tem traço nulo para todo $X \in \mathfrak{g}$. Isto implica que

$$
W(t, w)=0 \text { para todo } t \in \mathbb{R}
$$

ou seja, $w$ é ponto de equilíbrio de $W$.

Isto prova, em particular, que $e \in E_{W}$. Se $G$ não for semi-simples então $Z(G)$ é um subgrupo de Lie de $G$ de dimensão positiva, de acordo com o Teorema 2.17 - em particular, uma subvariedade -, de modo que toda vizinhança de e em $G$ contém pontos de $Z(G)$ e, portanto, de $E_{W}$. Com efeito, utilizando a estrutura de variedade de dimensão positiva de $Z(G)$, construímos uma sequência $\left(x_{n}\right)_{n \in \mathbb{N}}$ em $Z(G)$ que converge para $e$ na topologia de $Z(G)$ mas tal que $x_{n} \neq e$ para todo $n \in \mathbb{N}$. Esta construção pode ser feita tomando uma vizinhança coordenada de em $Z(G)$. Como a topologia de subvariedade de $Z(G)$ é necessariamente mais forte que a topologia de subespaço - ou, na melhor das hipóteses, igual - temos que $\left(x_{n}\right)_{n \in \mathbb{N}}$ converge para $e$ em $G$. Como pelo argumento anterior $Z(G) \subset E_{W}$, isto prova que $e$ não pode ser um ponto isolado de $E_{W}$.

É claro, contudo, que todo atrator local deve ser um equilíbrio isolado, de modo que se $G$ não é semi-simples então $e$ não pode ser um atrator local de $W$.

O próximo lema é muito mais geral do que necessitaremos no momento, o que pode deixar o leitor um tanto desmotivado. Esclarecemos que ele foi enunciado desta forma para ser usado também no Apêndice A, onde precisaremos dessa versão mais forte. 
Lema 4.10. Sejam $\Lambda^{0}: \mathbb{R} \rightarrow \mathfrak{g}$ uma curva suave e $\lambda: \mathbb{R} \rightarrow \mathfrak{g}$ dada por

$$
\lambda \doteq A d\left(\mathbf{x}_{\mathbf{r}}\right) \Lambda^{0}
$$

Então a n-ésima derivada de $\lambda$ é dada por

$$
\lambda^{(n)}=A d\left(\mathbf{x}_{\mathbf{r}}\right) \Lambda^{n}
$$

para todo $n \in \mathbb{N}$ onde $\Lambda^{n}: \mathbb{R} \rightarrow \mathfrak{g}$ é uma curva suave dada pela recorrência

$$
\Lambda^{n+1} \doteq\left(\Lambda^{n}\right)^{\prime}+\operatorname{ad}\left(X_{r}\right) \Lambda^{n}, n \in \mathbb{N}
$$

e $X_{r}$ é definido como em (3.3).

Demonstração. Por indução sobre $n$. O caso base $n=0$ é essencialmente a definição de $\lambda$. Observe que

$$
\operatorname{Ad}\left(\mathbf{x}_{\mathbf{r}}\right)^{\prime}=\operatorname{Ad}\left(\mathbf{x}_{\mathbf{r}}\right) \cdot \operatorname{ad}\left(X_{r}\right)
$$

o que pode ser constatado simplesmente derivando essa expressão:

$$
\begin{aligned}
\operatorname{Ad}\left(\mathbf{x}_{\mathbf{r}}\right)^{\prime} & =d \operatorname{Ad} \mathbf{x}_{\mathbf{r}}{ }^{\prime} \\
& =\operatorname{Ad}\left(\mathbf{x}_{\mathbf{r}}\right) \cdot \operatorname{ad}\left(d L_{\mathbf{x}_{\mathbf{r}}-1} \mathbf{x}_{\mathbf{r}}{ }^{\prime}\right) \\
& =\operatorname{Ad}\left(\mathbf{x}_{\mathbf{r}}\right) \cdot \operatorname{ad}\left(d L_{\mathbf{x}_{\mathbf{r}}-1} \sum_{j=1}^{m} u_{j}^{r} X_{j}\left(\mathbf{x}_{\mathbf{r}}\right)\right) \\
& =\operatorname{Ad}\left(\mathbf{x}_{\mathbf{r}}\right) \cdot \operatorname{ad}\left(\sum_{j=1}^{m} u_{j}^{r} X_{j}(e)\right) \\
& =\operatorname{Ad}\left(\mathbf{x}_{\mathbf{r}}\right) \cdot \operatorname{ad}\left(\sum_{j=1}^{m} u_{j}^{r} X_{j}\right) \\
& =\operatorname{Ad}\left(\mathbf{x}_{\mathbf{r}}\right) \cdot \operatorname{ad}\left(X_{r}\right) .
\end{aligned}
$$


Então se supusermos por indução que $\lambda^{(n)}=\operatorname{Ad}\left(\mathbf{x}_{\mathbf{r}}\right) \Lambda^{n}$ para algum $n \in \mathbb{N}$ teremos

$$
\begin{aligned}
\lambda^{(n+1)} & =\left(\lambda^{(n)}\right)^{\prime} \\
& =\left(\operatorname{Ad}\left(\mathbf{x}_{\mathbf{r}}\right) \Lambda^{n}\right)^{\prime} \\
& =\left(\operatorname{Ad}\left(\mathbf{x}_{\mathbf{r}}\right)\right)^{\prime} \Lambda^{n}+\operatorname{Ad}\left(\mathbf{x}_{\mathbf{r}}\right)\left(\Lambda^{n}\right)^{\prime} \\
& =\operatorname{Ad}\left(\mathbf{x}_{\mathbf{r}}\right) \operatorname{ad}\left(X_{r}\right) \Lambda^{n}+\operatorname{Ad}\left(\mathbf{x}_{\mathbf{r}}\right)\left(\Lambda^{n}\right)^{\prime} \\
& =\operatorname{Ad}\left(\mathbf{x}_{\mathbf{r}}\right)\left\{\left(\Lambda^{n}\right)^{\prime}+\operatorname{ad}\left(X_{r}\right) \Lambda^{n}\right\} \\
& =\operatorname{Ad}\left(\mathbf{x}_{\mathbf{r}}\right) \Lambda^{n+1}
\end{aligned}
$$

onde usamos (4.4) na quarta igualdade. Isto prova o passo de indução e encerra a prova.

Proposição 4.11. Seja $\mathbf{w}: \mathbb{R} \rightarrow G$ uma curva integral de $W$. Então a função $b_{k}: \mathbb{R} \rightarrow \mathbb{R}$ dada por

$$
b_{k}(t) \doteq \frac{d}{d t} a_{k}(t, \mathbf{w}(t))
$$

é limitada para cada $k=1, \ldots, m$.

Demonstração. Vamos obter explicitamente $b_{k}$, o que se resume a calcular as derivadas parciais de $a_{k}$ já que, pela regra da cadeia,

$$
b_{k}=\frac{\partial a_{k}}{\partial t}+\frac{\partial a_{k}}{\partial w} \mathbf{w}^{\prime}
$$

Recordando que

$$
\begin{aligned}
a_{k}(t, w) & =\operatorname{tr}\left\{\operatorname{Ad}(w) \cdot \operatorname{ad}\left(\operatorname{Ad}\left(\mathbf{x}_{\mathbf{r}}\right) X_{k}\right)\right\} \\
& =\operatorname{tr}\{\operatorname{Ad}(w) \cdot \operatorname{ad}(\lambda)\}
\end{aligned}
$$

onde $\lambda \doteq \operatorname{Ad}\left(\mathbf{x}_{\mathbf{r}}\right) X_{k}$ temos, por linearidade,

$$
\begin{aligned}
\frac{\partial a_{k}}{\partial t} & =\operatorname{tr}\left\{\operatorname{Ad}(w) \cdot \operatorname{ad}\left(\lambda^{\prime}\right)\right\} \\
& =\operatorname{tr}\left\{\operatorname{Ad}(w) \cdot \operatorname{ad}\left(\operatorname{Ad}\left(\mathbf{x}_{\mathbf{r}}\right) \operatorname{ad}\left(X_{r}\right) X_{k}\right)\right\} \\
& =\operatorname{tr}\left\{\operatorname{Ad}(w) \cdot \operatorname{Ad}\left(\mathbf{x}_{\mathbf{r}}\right) \cdot \operatorname{ad}\left(\operatorname{ad}\left(X_{r}\right) X_{k}\right) \cdot \operatorname{Ad}\left(\mathbf{x}_{\mathbf{r}}\right)^{-1}\right\}
\end{aligned}
$$

onde aplicamos o lema anterior para calcular $\lambda^{\prime}=\operatorname{Ad}\left(\mathbf{x}_{\mathbf{r}}\right) \operatorname{ad}\left(X_{r}\right) X_{k}$ - usando, na linguagem daquele lema, $\Lambda^{0} \doteq X_{k}$ - e a identidade (2.6) para obter a última igualdade. 
Quanto à derivada parcial de $a_{k}$ com relação à variável $w$, recordemos da relação (2.5):

$$
\begin{aligned}
d \operatorname{Ad}_{\mathbf{w}} \mathbf{w}^{\prime} & =\operatorname{Ad}(\mathbf{w}) \cdot \operatorname{ad}\left(d L_{\left.\mathbf{w}^{-1} \mathbf{w}^{\prime}\right)}\right. \\
& =\operatorname{Ad}(\mathbf{w}) \cdot \operatorname{ad}\left(d L_{\mathbf{w}^{-1}} \sum_{j=1}^{m} a_{j}(t, \mathbf{w}) \operatorname{Ad}\left(\mathbf{x}_{\mathbf{r}}\right) X_{j}(\mathbf{w})\right) \\
& =\operatorname{Ad}(\mathbf{w}) \cdot \sum_{j=1}^{m} a_{j}(t, \mathbf{w}) \operatorname{ad}\left(\operatorname{Ad}\left(\mathbf{x}_{\mathbf{r}}\right) X_{j}\right) \\
& =\operatorname{Ad}(\mathbf{w}) \cdot \operatorname{Ad}\left(\mathbf{x}_{\mathbf{r}}\right) \cdot \sum_{j=1}^{m} a_{j}(t, \mathbf{w}) \operatorname{ad}\left(X_{j}\right) \cdot \operatorname{Ad}\left(\mathbf{x}_{\mathbf{r}}\right)^{-1}
\end{aligned}
$$

onde usamos mais uma vez a identidade (2.6).

Assim

$$
\begin{aligned}
\frac{\partial a_{k}}{\partial w} \mathbf{w}^{\prime} & =\operatorname{tr}\left\{d \operatorname{Ad}_{\mathbf{w}} \mathbf{w}^{\prime} \cdot \operatorname{ad}\left(\operatorname{Ad}\left(\mathbf{x}_{\mathbf{r}}\right) X_{k}\right)\right\} \\
& =\operatorname{tr}\left\{\left(\operatorname{Ad}(\mathbf{w}) \cdot \operatorname{Ad}\left(\mathbf{x}_{\mathbf{r}}\right) \cdot \sum_{j=1}^{m} a_{j}(t, \mathbf{w}) \operatorname{ad}\left(X_{j}\right) \cdot \operatorname{Ad}\left(\mathbf{x}_{\mathbf{r}}\right)^{-1}\right) \cdot \operatorname{ad}\left(\operatorname{Ad}\left(\mathbf{x}_{\mathbf{r}}\right) X_{k}\right)\right\} \\
& =\operatorname{tr}\left\{\operatorname{Ad}(\mathbf{w}) \cdot \operatorname{Ad}\left(\mathbf{x}_{\mathbf{r}}\right) \cdot \sum_{j=1}^{m} a_{j}(t, \mathbf{w}) \operatorname{ad}\left(X_{j}\right) \cdot \operatorname{ad}\left(X_{k}\right) \cdot \operatorname{Ad}\left(\mathbf{x}_{\mathbf{r}}\right)^{-1}\right\}
\end{aligned}
$$

pois, novamente,

$$
\operatorname{ad}\left(\operatorname{Ad}\left(\mathbf{x}_{\mathbf{r}}\right) X_{k}\right)=\operatorname{Ad}\left(\mathbf{x}_{\mathbf{r}}\right) \cdot \operatorname{ad}\left(X_{k}\right) \cdot \operatorname{Ad}\left(\mathbf{x}_{\mathbf{r}}\right)^{-1}
$$

Somando as duas parcelas calculadas no ponto $(t, \mathbf{w}(t))$, concluímos que

$$
b_{k}=\operatorname{tr}\left\{\operatorname{Ad}(\mathbf{w}) \cdot \operatorname{Ad}\left(\mathbf{x}_{\mathbf{r}}\right) \cdot B_{k} \cdot \operatorname{Ad}\left(\mathbf{x}_{\mathbf{r}}\right)^{-1}\right\}
$$

onde $B_{k}: \mathbb{R} \rightarrow \mathfrak{g l}(\mathfrak{g})$ é dada por

$$
B_{k} \doteq \operatorname{ad}\left(\operatorname{ad}\left(X_{r}\right) X_{k}\right)+\sum_{j=1}^{m} a_{j}(t, \mathbf{w}) \operatorname{ad}\left(X_{j}\right) \cdot \operatorname{ad}\left(X_{k}\right)
$$

Denotemos por $\|\cdot\|$ uma norma em $\mathfrak{g l}(\mathfrak{g})$, cuja natureza é irrelevante. Recordando que a representação adjunta

$$
\mathrm{Ad}: G \rightarrow \mathrm{GL}(\mathfrak{g}) \subset \mathfrak{g l}(\mathfrak{g})
$$

é contínua (pois é suave) e que $G$ é por hipótese compacto, temos que a imagem de Ad é um 
subconjunto compacto e, portanto, limitado de $\mathfrak{g l}(\mathfrak{g})$. Ou seja, existe $M>0$ tal que

$$
\|\operatorname{Ad}(x)\| \leq M \text { para todo } x \in G \text {. }
$$

Daí para $t \in \mathbb{R}$ temos que

$$
\begin{aligned}
\left|b_{k}(t)\right| & =\left|\operatorname{tr}\left\{\operatorname{Ad}(\mathbf{w}) \cdot \operatorname{Ad}\left(\mathbf{x}_{\mathbf{r}}\right) \cdot B_{k}(t) \cdot \operatorname{Ad}\left(\mathbf{x}_{\mathbf{r}}\right)^{-1}\right\}\right| \\
& \leq\|\operatorname{tr}\|\left\|\operatorname{Ad}(\mathbf{w}) \cdot \operatorname{Ad}\left(\mathbf{x}_{\mathbf{r}}\right) \cdot B_{k}(t) \cdot \operatorname{Ad}\left(\mathbf{x}_{\mathbf{r}}\right)^{-1}\right\| \\
& \leq\|\operatorname{tr}\|\|\operatorname{Ad}(\mathbf{w})\|\left\|\operatorname{Ad}\left(\mathbf{x}_{\mathbf{r}}\right)\right\|\left\|B_{k}(t)\right\|\left\|\operatorname{Ad}\left(\mathbf{x}_{\mathbf{r}}\right)^{-1}\right\| \\
& =\|\operatorname{tr}\|\|\operatorname{Ad}(\mathbf{w})\|\left\|\operatorname{Ad}\left(\mathbf{x}_{\mathbf{r}}\right)\right\|\left\|B_{k}(t)\right\|\left\|\operatorname{Ad}\left(\mathbf{x}_{\mathbf{r}}{ }^{-1}\right)\right\| \\
& \leq M^{3}\|\operatorname{tr}\|\left\|B_{k}(t)\right\|
\end{aligned}
$$

onde $\|\operatorname{tr}\|$ é a norma do traço como transformação linear contínua $\operatorname{tr}: \mathfrak{g l}(\mathfrak{g}) \rightarrow \mathbb{R}$. Isto prova que se $B_{k}$ é limitada então $b_{k}$ também o é.

Para provar que $B_{k}$ é limitada note que

$$
\begin{aligned}
\left\|B_{k}(t)\right\| & =\left\|\operatorname{ad}\left(\operatorname{ad}\left(X_{r}(t)\right) X_{k}\right)+\sum_{j=1}^{m} a_{j}(t, \mathbf{w}) \operatorname{ad}\left(X_{j}\right) \cdot \operatorname{ad}\left(X_{k}\right)\right\| \\
& \leq\left\|\operatorname{ad}\left(\operatorname{ad}\left(X_{r}(t)\right) X_{k}\right)\right\|+\sum_{j=1}^{m}\left|a_{j}(t, \mathbf{w})\right|\left\|\operatorname{ad}\left(X_{j}\right) \cdot \operatorname{ad}\left(X_{k}\right)\right\|
\end{aligned}
$$

para todo $t \in \mathbb{R}$. A primeira parcela acima é limitada pois a aplicação

$$
t \in \mathbb{R} \mapsto \operatorname{ad}\left(\operatorname{ad}\left(X_{r}(t)\right) X_{k}\right) \in \mathfrak{g l}(\mathfrak{g})
$$

é $T$-periódica, já que $X_{r}$ é definido por $(3.3)$ e $u_{1}^{r}, \ldots, u_{m}^{r}$ são funções $T$-periódicas. Logo, para provar que $B_{k}$ é limitada, basta mostrar que a segunda parcela acima é limitada, e para isto é suficiente mostrar que a aplicação

$$
t \in \mathbb{R} \mapsto a_{j}(t, \mathbf{w}(t)) \in \mathbb{R}
$$

é limitada para cada $j=1, \ldots, m$.

Observemos que, segundo a própria definição (3.10), cada $a_{j}: \mathbb{R} \times G \rightarrow \mathbb{R}$ é $T$-periódica i.e. para cada $w \in G$ fixado a aplicação

$$
t \in \mathbb{R} \mapsto a_{j}(t, w) \in \mathbb{R}
$$

é $T$-periódica. Denotando

$$
K \doteq\left\{a_{j}(t, w) ; t \in[0, T], w \in G\right\}
$$


isto implica que $a_{j}(t, w) \in K$ para todo par $(t, w) \in \mathbb{R} \times G$. Contudo, $K \subset \mathbb{R}$ é compacto pois é a imagem por $a_{j}$ - que é uma aplicação contínua - do conjunto $[0, T] \times G$, o qual é compacto (aqui usamos a compacidade de $G$ ). Isto implica que $a_{j}$ é limitada, o que conclui nossa demonstração de que $B_{k}$ é limitada, e que por sua vez implica que $b_{k}$ é limitada.

Para demonstrar o próximo resultado, precisaremos do Lema de Barbalat e de um importante corolário seu, os quais demonstramos abaixo.

Lema 4.12 (Barbalat). Seja $\beta: \mathbb{R}_{+} \rightarrow \mathbb{R}$ uma função uniformemente contínua tal que o limite

$$
\int_{0}^{\infty} \beta(t) d t \doteq \lim _{x \rightarrow \infty} \int_{0}^{x} \beta(t) d t
$$

existe e é finito. Então

$$
\lim _{t \rightarrow \infty} \beta(t)=0 .
$$

Demonstração. Suponhamos por absurdo a tese é falsa: existe $\epsilon>0$ tal que para cada $M>0$ existe $t_{M} \geq M$ tal que

$$
\left|\beta\left(t_{M}\right)\right| \geq \epsilon
$$

Podemos então construir uma sequência $\left(t_{n}\right)_{n \in \mathbb{N}}$ tal que $t_{n} \geq n$ e $\left|\beta\left(t_{n}\right)\right| \geq \epsilon$ para todo $n \in \mathbb{N}$. Observe que, nestas condições, $\lim _{n \rightarrow \infty} t_{n}=\infty$.

Como $\beta$ é uniformemente contínua existe $\delta>0$ tal que

$$
\left|t-t_{n}\right|<\delta \Rightarrow\left|\beta(t)-\beta\left(t_{n}\right)\right|<\frac{\epsilon}{2}
$$

para quaisquer $t \geq 0$ e $n \in \mathbb{N}$. Em particular, se $\left|t-t_{n}\right|<\delta$ temos que

$$
|\beta(t)| \geq\left|\beta\left(t_{n}\right)\right|-\left|\beta(t)-\beta\left(t_{n}\right)\right|>\epsilon-\frac{\epsilon}{2}=\frac{\epsilon}{2} .
$$

Observe que a desigualdade acima implica, em particular, que $\beta$ não se anula no intervalo $\left[t_{n}, t_{n}+\delta\right]$ e, sendo contínua, não muda de sinal nesse intervalo. É fácil ver que, sendo esse o caso, tem-se

$$
\int_{t_{n}}^{t_{n}+\delta}|\beta(t)| d t=\left|\int_{t_{n}}^{t_{n}+\delta} \beta(t) d t\right|
$$

o que vale para todo $n \in \mathbb{N}$. 
Das observações acima deduzimos que

$$
\begin{aligned}
\left|\int_{0}^{t_{n}+\delta} \beta(t) d t-\int_{0}^{t_{n}} \beta(t) d t\right| & =\left|\int_{t_{n}}^{t_{n}+\delta} \beta(t) d t\right| \\
& =\int_{t_{n}}^{t_{n}+\delta}|\beta(t)| d t \\
& \geq \int_{t_{n}}^{t_{n}+\delta} \frac{\epsilon}{2} d t \\
& =\frac{\epsilon \delta}{2}
\end{aligned}
$$

para todo $n \in \mathbb{N}$. Contudo

$$
\lim _{n \rightarrow \infty}\left|\int_{0}^{t_{n}+\delta} \beta(t) d t-\int_{0}^{t_{n}} \beta(t) d t\right|=\left|\int_{0}^{\infty} \beta(t) d t-\int_{0}^{\infty} \beta(t) d t\right|=0
$$

o que contradiz a limitação inferior obtida.

Corolário 4.13. Suponha que $\alpha: \mathbb{R}_{+} \rightarrow \mathbb{R}$ é uma função de classe $C^{2}$ tal que o limite

$$
\lim _{t \rightarrow \infty} \alpha(t)
$$

existe e é finito e que, ademais, $\alpha^{\prime \prime}$ é limitada. Então

$$
\lim _{t \rightarrow \infty} \alpha^{\prime}(t)=0
$$

Demonstração. Segue do Lema de Barbalat aplicado à função $\beta \doteq \alpha^{\prime}$, bastando para isto que provemos que $\alpha^{\prime}$ é uniformemente contínua pois, neste caso,

$$
\lim _{x \rightarrow \infty} \int_{0}^{x} \alpha^{\prime}(t) d t=\lim _{x \rightarrow \infty} \alpha(x)-\alpha(0)
$$

existe e é finito por hipótese. Porém, dados $t_{1}, t_{2} \in \mathbb{R}$ distintos temos, do Teorema do Valor Médio, que existe $s$ pertencente ao intervalo limitado definido por $t_{1}$ e $t_{2}$ tal que

$$
\left|\alpha^{\prime}\left(t_{1}\right)-\alpha^{\prime}\left(t_{2}\right)\right|=\left|\alpha^{\prime \prime}(s)\left(t_{1}-t_{2}\right)\right| \leq\left|\alpha^{\prime \prime}(s)\right|\left|t_{1}-t_{2}\right|
$$

Usando agora a hipótese de que $\alpha^{\prime \prime}$ é limitada concluímos que $\alpha^{\prime}$ é uniformemente contínua.

Proposição 4.14. Seja $\mathbf{w}: \mathbb{R} \rightarrow G$ uma curva integral de $W$. Então

$$
\lim _{t \rightarrow \infty} a_{k}(t, \mathbf{w}(t))=0
$$

para $k=1, \ldots, m$. 
Demonstração. Defina $\alpha: \mathbb{R} \rightarrow \mathbb{R}$ por

$$
\alpha \doteq V \circ \mathbf{w}
$$

onde $V$ é nossa função "tipo Lyapunov" (3.8). Temos

$$
\begin{aligned}
\alpha^{\prime} & =\frac{d}{d t} V(\mathbf{w}) \\
& =d V\left(\mathbf{w}^{\prime}\right) \\
& =d V\left(\sum_{k=1}^{m} a_{k}(t, \mathbf{w}) \operatorname{Ad}\left(\mathbf{x}_{\mathbf{r}}\right) X_{k}(\mathbf{w})\right) \\
& =\sum_{k=1}^{m} a_{k}(t, \mathbf{w}) d V\left(\operatorname{Ad}\left(\mathbf{x}_{\mathbf{r}}\right) X_{k}(\mathbf{w})\right) \\
& =\sum_{k=1}^{m} a_{k}(t, \mathbf{w})^{2}
\end{aligned}
$$

onde a última igualdade segue da definição de $a_{k}$ (3.10). Derivando mais uma vez temos

$$
\begin{aligned}
\alpha^{\prime \prime} & =\frac{d}{d t} \sum_{k=1}^{m} a_{k}(t, \mathbf{w})^{2} \\
& =2 \sum_{k=1}^{m} a_{k}(t, \mathbf{w}) \frac{d}{d t} a_{k}(t, \mathbf{w}) \\
& =2 \sum_{k=1}^{m} a_{k}(t, \mathbf{w}) b_{k}(t)
\end{aligned}
$$

onde $b_{k}$ é como na Proposição 4.11, a qual afirma que $b_{k}$ é limitada para cada $k=1, \ldots, m$. Como $a_{k}$ também é limitada para cada $k=1, \ldots, m$ (pois é $T$-periódica e $G$ é compacto, como pode ser visto em mais detalhes na própria demonstração da Proposição 4.11) temos que $\alpha^{\prime \prime}$ é limitada.

Temos

$$
d V(W(t, w))=\sum_{k=1}^{m} a_{k}(t, w)^{2} \geq 0
$$

para todo par $(t, w) \in \mathbb{R} \times G$, de modo que $V$ é não decrescente ao longo de $W$ conforme a Definição 4.2. Segue então do Corolário 4.4 que

$$
\lim _{t \rightarrow \infty} V(\mathbf{w}(t))=V(w)
$$

onde $w \in \Omega_{W}(0, \mathbf{w}(0))$ é arbitrário. Denotando o valor acima por $\alpha_{0}$ temos que

$$
\lim _{t \rightarrow \infty} \alpha(t)=\alpha_{0}
$$


Juntando a última conclusão aos fatos demonstrados previamente de que $\alpha^{\prime}$ é não negativa e $\alpha^{\prime \prime}$ é limitada concluímos, pelo Corolário 4.13 do Lema de Barbalat, que

$$
\lim _{t \rightarrow \infty} \alpha^{\prime}(t)=0
$$

Recordando a expressão de $\alpha^{\prime}$ temos

$$
\lim _{t \rightarrow \infty} \sum_{k=1}^{m} a_{k}(t, \mathbf{w}(t))^{2}=0
$$

de modo que, necessariamente,

$$
\lim _{t \rightarrow \infty} a_{k}(t, \mathbf{w}(t))=0
$$

para $k=1, \ldots, m$.

Lema 4.15. Considere $G$ munido de uma métrica bi-invariante, cuja norma induzida em cada espaço tangente denotaremos por $\|\cdot\|$. Se $\mathbf{w}: \mathbb{R} \rightarrow G$ é curva integral do campo auxiliar $W$ então

$$
\lim _{t \rightarrow \infty}\left\|\mathbf{w}^{\prime}(t)\right\|=0
$$

Demonstração. É só observar que

$$
\begin{aligned}
\left\|\mathbf{w}^{\prime}(t)\right\| & =\left\|\sum_{k=1}^{m} a_{k}(t, \mathbf{w}) \operatorname{Ad}\left(\mathbf{x}_{\mathbf{r}}\right) X_{k}(\mathbf{w})\right\| \\
& \leq \sum_{k=1}^{m}\left|a_{k}(t, \mathbf{w})\right|\left\|\operatorname{Ad}\left(\mathbf{x}_{\mathbf{r}}\right) X_{k}(\mathbf{w})\right\| \\
& =\sum_{k=1}^{m}\left|a_{k}(t, \mathbf{w})\right|\left\|\operatorname{Ad}\left(\mathbf{x}_{\mathbf{r}}\right) X_{k}\right\| \\
& =\sum_{k=1}^{m}\left|a_{k}(t, \mathbf{w})\right|\left\|X_{k}\right\|
\end{aligned}
$$

para todo $t \in \mathbb{R}$, onde usamos a propriedade de que toda métrica bi-invariante em $G$ advém de um produto interno Ad-invariante em $\mathfrak{g}$, conforme o comentário que sucede a Proposição 2.15. A conclusão segue da Proposição 4.14.

Já estamos em condições de demonstrar o Teorema 4.7. 
Demonstração do Teorema 4.7. Primeiramente, introduzimos uma métrica bi-invariante em $G$, a qual denotamos simplificadamente por $\|\cdot\|$. Seja $\mathbf{w}: \mathbb{R} \rightarrow G$ a única curva integral de $W$ que satisfaz $\mathbf{w}\left(t_{0}\right)=w_{0}$. Do Lema 4.15 temos que

$$
\lim _{t \rightarrow \infty}\left\|\mathbf{w}^{\prime}(t)\right\|=0
$$

Definimos $f: \mathbb{R} \times G \rightarrow \mathbb{R}$ por

$$
f(t, w) \doteq a_{k}(t, w)
$$

a qual é T-periódica e satisfaz, segundo a Proposição 4.14,

$$
\lim _{t \rightarrow \infty} f(t, \mathbf{w}(t))=\lim _{t \rightarrow \infty} a_{k}(t, \mathbf{w})=0 .
$$

Da definição de ponto $\Omega$-limite, se $w \in \Omega_{W}\left(t_{0}, w_{0}\right)$ então existe uma sequência $\left(t_{n}\right)_{n \in \mathbb{N}}$ em $\mathbb{R}$, crescente e ilimitada superiormente, tal que

$$
\lim _{n \rightarrow \infty} \mathbf{w}\left(t_{n}\right)=w
$$

A digressão acima garante válidas as hipóteses necessárias sobre $\mathbf{w}, f$ e $w$ para aplicarmos a Proposição 4.6, a qual nos garante nestas condições que

$$
f(t, w)=0 \text { para todo } t \in \mathbb{R} .
$$

Ou seja, $a_{k}(t, w)=0$ para todo $t \in \mathbb{R}$.

A primeira consequência notável do Teorema 4.7 é que o conjunto dos pontos de equilíbrio $E_{W}$ do campo auxiliar coincide com o conjunto de todos os seus pontos $\Omega$-limite

$$
\Omega_{W} \doteq \bigcup_{\left(t_{0}, w_{0}\right)} \Omega_{W}\left(t_{0}, w_{0}\right)
$$

Com efeito, é óbvio da definição de conjunto $\Omega$-limite que $E_{W} \subset \Omega_{W}$. A outra inclusão segue imediatamente do teorema anterior.

Uma consequência da discussão acima é o seguinte corolário, o qual não utilizaremos nas próximas seções, mas aprofunda a intuição geométrica a respeito do comportamento das curvas integrais do campo auxiliar.

Corolário 4.16. Se $\mathbf{w}: \mathbb{R} \rightarrow G$ é uma curva integral de $W$ então

$$
\mathbf{w}(t) \stackrel{t \rightarrow \infty}{\longrightarrow} E_{W}
$$

i.e. se $U \subset G$ é uma vizinhança de $E_{W}$ então existe $t_{U} \in \mathbb{R}$ tal que $\mathbf{w}(t) \in U$ para todo $t \geq t_{U}$. 
Demonstração. Supondo, por absurdo, que a tese é falsa, encontramos uma vizinhança $U_{0} \subset G$ de $E_{W}$ com a seguinte propriedade: para cada $n \in \mathbb{N}$ existe $t_{n} \geq n$ tal que

$$
\mathbf{w}\left(t_{n}\right) \notin U_{0} .
$$

Podemos supor, sem perda de generalidade, que $\left(t_{n}\right)_{n \in \mathbb{N}}$ é uma sequência crescente, a qual é ilimitada superiormente por construção. Como $G$ é compacto, esta sequência possui uma subsequência, a qual denotamos por $\left(t_{n_{k}}\right)_{k \in \mathbb{N}}$, tal que a sequência $\left(\mathbf{w}\left(t_{n_{k}}\right)\right)_{k \in \mathbb{N}}$ é convergente em $G$, digamos, para um ponto $w \in G$. Como necessariamente a subsequência $\left(t_{n_{k}}\right)_{k \in \mathbb{N}}$ também é crescente e ilimitada superiormente, concluímos que $w \in \Omega_{W}$ por definição de ponto $\Omega$-limite.

A discussão que precede o enunciado implica que $\Omega_{W}=E_{W}$, de modo que $w \in E_{W}$. Porém

$$
\lim _{k \rightarrow \infty} \mathbf{w}\left(t_{n_{k}}\right)=w
$$

o que por sua vez implica que toda vizinhança de $w$ contém pontos da forma $\mathbf{w}\left(t_{n_{k}}\right)$ para algum $k \in \mathbb{N}$. Isto contradiz nossa hipótese, pois $U_{0}$, sendo vizinhança de $E_{W}$, é também vizinhança de $w$, mas não satisfaz essa propriedade.

\subsection{Comentários e referências}

Os resultados e conceitos preliminares introduzidos na Seção 4.1 são, com algumas diferenças de abordagem, da teoria de estabilidade de Lyapunov. Encontramos leituras inspiradoras a respeitos desses tópicos em [Vid02], Capítulo 5, e [BL05], Capítulo 6. O objetivo principal dessa seção foi mimetizar, no nosso contexto abstrato, os resultados encontrados em [Sil09], Apêndice E. O Lema 4.5 é nossa versão adaptada de [Sil09], Lema E.4, página 173. Já a Proposição 4.6 é nossa versão, bastante abstrata, de [Sil09], Lemas E.5 e E.6, páginas 173-177: sua demonstração é inspirada na dos referidos lemas e na do Princípio da Invariância de LaSalle (veja [Vid02], Capítulo 5, Lemma 71, páginas 177 e 178).

O Teorema 4.7, a Proposição 4.14 e o Corolário 4.16 são análogos aos resultados encontrados em [Sil09], Teorema 4.11, páginas 91-93, e Teorema 4.16, páginas 98-103, com o conjunto $E_{W}$ fazendo o papel dos conjuntos $E_{R}$ e $E_{C}$, definidos nas páginas 91 e 98 , respectivamente, da supracitada referência. As técnicas que empregamos nas suas demonstrações ao longo da Seção 4.2 são, embora análogas nas ideias principais, são distintas daquelas em vários pontos e aspectos técnicos.

Uma diferença fundamental é que trabalhamos exclusivamente com trajetórias de referência suaves (Definição 3.1), em contraste com a abordagem de [Sil09] que trabalha com trajetórias de referência suaves por partes. Essa restrição, além de simplificar muitas contas e estimativas e, portanto, toda a parte técnica da demonstração, permitiu que demonstrássemos o resultado sem ter uma descrição explícita da trajetória de referência: utilizamos apenas suavidade e periodicidade, tanto da curva quanto dos controles. Acreditamos que essa é uma melhoria substancial na teoria e que justifica a concessão feita.

Ainda quanto a estes dois resultados, recapitulemos a discussão iniciada na Seção 3.3 sobre o 
caso particular $G=\mathrm{SU}(n)$. Neste caso a Proposição 4.14 nos diz que se $\mathbf{w}: \mathbb{R} \rightarrow \mathrm{SU}(n)$ é uma curva integral do campo auxiliar

$$
W(t, w)=w \cdot \mathbf{x}_{\mathbf{r}}(t) \cdot \sum_{k=1}^{m} a_{k}(t, w) X_{k} \cdot \mathbf{x}_{\mathbf{r}}(t)^{*}
$$

então

$$
\lim _{t \rightarrow \infty} a_{k}(t, \mathbf{w}(t))=\lim _{t \rightarrow \infty} d V\left(\mathbf{w}(t) \cdot \mathbf{x}_{\mathbf{r}}(t) \cdot X_{k} \cdot \mathbf{x}_{\mathbf{r}}(t)^{*}\right)=0
$$

para todo $k=1, \ldots, m$. Já o Teorema 4.7 nos diz que se $w \in \mathrm{SU}(n)$ é um ponto $\Omega$-limite do campo auxiliar então

$$
a_{k}(t, w)=d V\left(w \cdot \mathbf{x}_{\mathbf{r}}(t) \cdot X_{k} \cdot \mathbf{x}_{\mathbf{r}}(t)^{*}\right)=0
$$

para qualquer $t \in \mathbb{R}$ e todo $k=1, \ldots, m$. Compare estas expressões com aquelas apresentadas em [Sil09], Teorema 4.11, páginas 91-93, e Teorema 4.16, páginas 98-103.

Alguns comentários se fazem necessários a respeito da Proposição 4.11. Embora estejamos supondo $G$ compacto por simplicidade - e de fato esta hipótese se tornará cada vez mais relevante no decorrer do presente trabalho - esta hipótese não é fundamental para sua demonstração. Tampouco utilizamos a periodicidade de $\left(\mathbf{x}_{\mathbf{r}}, u_{1}^{r}, \ldots, u_{m}^{r}\right)$ de forma plena. De fato, recapitulando os passos da demonstração, os pontos essenciais foram listados abaixo.

- Limitação da representação adjunta de $G$. De fato, apesar de termos usado a compacidade de $G$ para provar (4.5), poderíamos apenas ter incluído esta limitação como hipótese. Um jeito de garanti-la seria encontrando um produto interno Ad-invariante em $\mathfrak{g}$ - i.e. uma métrica bi-invariante em $G$ - pois, nesse caso, teríamos

$$
\|\operatorname{Ad}(x)\|=1 \text { para todo } x \in G
$$

Claro que um jeito de garantir a existência de um tal produto interno é, segundo a Proposição 2.15 , supondo que $G$ é compacto...

- Limitação da aplicação

$$
t \in \mathbb{R} \mapsto \operatorname{ad}\left(\operatorname{ad}\left(X_{r}(t)\right) X_{k}\right) \in \mathfrak{g l}(\mathfrak{g})
$$

Aqui, não foi fundamental a periodicidade de $X_{r}$, apenas sua limitação - a qual é equivalente à limitação conjunta dos controles $u_{1}^{r}, \ldots, u_{m}^{r}$.

- Limitação de cada $a_{j}$. Esta também não depende nem da compacidade de $G$ nem da periodicidade. De fato, podemos limitar $a_{j}$ usando (4.3) e supondo que Ad é limitada.

Também na Proposição 4.14 não foram fundamentais a compacidade de $G$ nem a periodicidade 
de $\left(\mathbf{x}_{\mathbf{r}}, u_{1}^{r}, \ldots, u_{m}^{r}\right)$. Utilizamos apenas que a trajetória $\mathbf{w}$ está definida em $\mathbb{R}$, que seu conjunto $\Omega$-limite é não vazio e que $\mathbf{x}_{\mathbf{r}}$ está definida em $\mathbb{R}$. Observações análogas a estas podem ser feitas a respeito de outros resultados do presente capítulo.

O enunciado e a demonstração do Lema de Barbalat (Lema 4.12) foram extraídos de [Kha02], Lemma 8.2, página 323. Seu Corolário 4.13 e a respectiva demonstração foram imitados de [Sil09], Lema 4.9, páginas 90 e 91. 


\section{Capítulo 5}

\section{Trajetórias regulares}

Até aqui, todas as construções e resultados que obtivemos são válidos para qualquer trajetória de referência periódica do sistema (3.1) em um grupo de Lie compacto e conexo. Neste capítulo estudamos uma classe especial de trajetórias de referência periódicas, as quais denominamos regulares e que definimos a seguir. Esta classe de trajetórias admite uma descrição algébrica do conjunto dos pontos de equilíbrio de seu campo auxiliar associado (Proposição 5.3): ele coincide com o conjunto dos pontos críticos da nossa função "tipo Lyapunov" i.e. o caractere da representação adjunta de $G$. Esta caracterização permite, sob essas hipóteses, mostrar que a identidade é um atrator local do campo auxiliar associado à trajetória de referência se $G$ for semi-simples (Teorema 5.11) e, portanto, resolver o problema do planejamento periódico de trajetórias em uma vizinhança do estado objetivo $x_{\infty}$ conforme discutido na Proposição 3.6.

Neste capítulo $G$ é um grupo de Lie compacto e conexo cuja álgebra de Lie denotamos por $\mathfrak{g}$. Os campos invariantes à esquerda $X_{1}, \ldots, X_{m} \in \mathfrak{g}$ estão fixados como na Seção 3.1. Denotaremos sempre por $V$ nossa função "tipo Lyapunov" (3.8).

\subsection{Trajetórias regulares}

Conforme notação da Seção 3.1 denotamos

$$
\Gamma \doteq \operatorname{span}\left\{X_{1}, \ldots, X_{m}\right\}
$$

Definição 5.1. Uma trajetória $\left(\mathbf{x}, u_{1}, \ldots, u_{m}\right)$ do sistema $(3.1)$ - não necessariamente uma trajetória de referência periódica - é dita trajetória regular se

$$
\operatorname{span}\left\{\operatorname{Ad}(\mathbf{x}(t)) X_{k} ; t \in \mathbb{R}, 1 \leq k \leq m\right\}=\operatorname{Lie}(\Gamma) .
$$

Observe que como estamos supondo que $\Gamma$ é Lie-determinada então $\left(\mathbf{x}, u_{1}, \ldots, u_{m}\right)$ é trajetória regular do sistema (3.1) se e somente se

$$
\operatorname{span}\left\{\operatorname{Ad}(\mathbf{x}(t)) X_{k} ; t \in \mathbb{R}, 1 \leq k \leq m\right\}=\mathfrak{g}
$$

Manteremos esta hipótese consistentemente no decorrer deste capítulo. No Apêndice A construímos de maneira mais ou menos explícita uma trajetória de referência $T$-periódica, que é regular sempre 
que o sistema (3.1) satisfizer algumas hipóteses adicionais que são independentes do fato de $\Gamma$ ser Lie-determinada ou não.

O Teorema 4.7 admite o seguinte

Corolário 5.2. Suponha que $\left(\mathbf{x}_{\mathbf{r}}, u_{1}^{r}, \ldots, u_{m}^{r}\right)$ é uma trajetória de referência T-periódica do sistema (3.1) que é regular, e seja $W$ o campo auxiliar (3.9) associado a esta trajetória. Nessas condições, se $w \in \Omega_{W}\left(t_{0}, w_{0}\right)$ para algum $\left(t_{0}, w_{0}\right) \in \mathbb{R} \times G$ então

$$
\operatorname{tr}\{\operatorname{Ad}(w) \cdot \operatorname{ad}(X)\}=0
$$

para todo $X \in \mathfrak{g}$. Ou seja

$$
d V X(w)=0 \text { para todo } X \in \mathfrak{g} .
$$

Demonstração. Segundo o Teorema 4.7 temos, para cada $k=1, \ldots, m$,

$$
a_{k}(t, w)=0 \text { para todo } t \in \mathbb{R}
$$

ou seja

$$
\operatorname{tr}\left\{\operatorname{Ad}(w) \cdot \operatorname{ad}\left(\operatorname{Ad}\left(\mathbf{x}_{\mathbf{r}}(t)\right) X_{k}\right)\right\}=0 \text { para todo } t \in \mathbb{R}
$$

Mas se $\left(\mathbf{x}_{\mathbf{r}}, u_{1}^{r}, \ldots, u_{m}^{r}\right)$ é regular então

$$
\operatorname{span}\left\{\operatorname{Ad}\left(\mathbf{x}_{\mathbf{r}}(t)\right) X_{k} ; t \in \mathbb{R}, 1 \leq k \leq m\right\}=\mathfrak{g}
$$

de modo que, por linearidade, temos

$$
\operatorname{tr}\{\operatorname{Ad}(w) \cdot \operatorname{ad}(X)\}=0
$$

para todo $X \in \mathfrak{g}$.

Em particular se $\left(\mathbf{x}_{\mathbf{r}}, u_{1}^{r}, \ldots, u_{m}^{r}\right)$ é trajetória de referência $T$-periódica regular do sistema (3.1) então o conjunto dos pontos de equilíbrio (3.11) do campo auxiliar $W$ associado a essa trajetória pode ser expresso por

$$
E_{W}=\{w \in G ; \operatorname{tr}\{\operatorname{Ad}(w) \cdot \operatorname{ad}(X)\}=0 \text { para todo } X \in \mathfrak{g}\}
$$

De fato, concluímos no fim da Seção 4.2 que $E_{W}=\Omega_{W}$, onde $\Omega_{W}$ é a reunião de todos os pontos $\Omega$-limite de $W$, definido por (4.6). Logo, se $w \in E_{W}$ então pelo corolário anterior temos

$$
\operatorname{tr}\{\operatorname{Ad}(w) \cdot \operatorname{ad}(X)\}=0
$$


para todo $X \in \mathfrak{g}$. Reciprocamente, se $w \in G$ satisfaz a equação anterior então é claro da expressão (4.3) para $a_{k}$ que

$$
a_{k}(t, w)=0 \text { para todo } t \in \mathbb{R}
$$

para cada $k=1, \ldots, m$, de modo que

$$
W(t, w)=0 \text { para todo } t \in \mathbb{R}
$$

i.e. $w \in E_{W}$. Observe ainda que o conjunto descrito em (5.1) é, conforme a equação (4.2), exatamente o conjunto dos pontos críticos de $V$ i.e. o conjunto dos pontos de $G$ onde o diferencial de $V$ se anula.

Sintetizamos nossas conclusões até agora no seguinte enunciado.

Proposição 5.3. Suponha que $\left(\mathbf{x}_{\mathbf{r}}, u_{1}^{r}, \ldots, u_{m}^{r}\right)$ é uma trajetória de referência T-periódica regular do sistema (3.1), e seja $W$ o campo auxiliar associado a esta trajetória. Então são iguais os seguintes conjuntos:

- o conjunto dos pontos de equilíbrio $E_{W}$;

- o conjunto dos pontos $\Omega$-limite $\Omega_{W}$;

- o conjunto dos pontos críticos de $V$.

Até o fim deste capítulo suporemos que $\left(\mathbf{x}_{\mathbf{r}}, u_{1}^{r}, \ldots, u_{m}^{r}\right)$ é regular no sentido da Definição 5.1.

\subsection{Estudo dos conjuntos $\Omega$-limite - parte II}

Recapitulando nossos objetivos postos no fim do Capítulo 3, já demonstramos no Corolário 4.9 que $e \in E_{W}$. Este mesmo corolário nos diz que o nosso segundo objetivo - provar que é um atrator local de $W$ - só é factível se $G$ for um grupo de Lie semi-simples. Ainda sem supor esta condição, vamos deduzir mais algumas propriedades do conjunto $E_{W}$ que nos aproximarão da solução do problema.

\section{Proposição 5.4.}

1. Se $x \in E_{W}$ e $y \in G$ então $y \cdot x \cdot y^{-1} \in E_{W}$.

2. Se $x \cdot y \in E_{W}$ então $y \cdot x \in E_{W}$.

3. Se $x \in E_{W}$ então $x^{-1} \in E_{W}$.

Demonstração. Por aplicação de (5.1). 
1. Para todo $X \in \mathfrak{g}$ temos que

$$
\begin{aligned}
\operatorname{tr}\left\{\operatorname{Ad}\left(y \cdot x \cdot y^{-1}\right) \cdot \operatorname{ad}(X)\right\} & =\operatorname{tr}\left\{\operatorname{Ad}(y) \cdot \operatorname{Ad}(x) \cdot \operatorname{Ad}(y)^{-1} \cdot \operatorname{ad}(X)\right\} \\
& =\operatorname{tr}\left\{\operatorname{Ad}(x) \cdot \operatorname{Ad}(y)^{-1} \cdot \operatorname{ad}(X) \cdot \operatorname{Ad}(y)\right\} \\
& =\operatorname{tr}\left\{\operatorname{Ad}(x) \cdot \operatorname{ad}\left(\operatorname{Ad}(y)^{-1} X\right)\right\} \\
& =0
\end{aligned}
$$

pois $x \in E_{W}$ por hipótese. Daí $y \cdot x \cdot y^{-1} \in E_{W}$.

2. Se $x \cdot y$ pertence a $E_{W}$ então pelo item anterior temos que $y \cdot(x \cdot y) \cdot y^{-1}=y \cdot x$ também pertence a $E_{W}$.

3. Sejam $\langle\cdot, \cdot\rangle$ um produto interno Ad-invariante em $\mathfrak{g}$ conforme a Proposição 2.15 e $Y_{1}, \ldots, Y_{n}$ uma base ortonormal de $\mathfrak{g}$. Então fixado $X \in \mathfrak{g}$ temos que

$$
\begin{aligned}
\operatorname{tr}\left\{\operatorname{Ad}\left(x^{-1}\right) \cdot \operatorname{ad}(X)\right\} & =\sum_{j=1}^{n}\left\langle\operatorname{Ad}\left(x^{-1}\right) \cdot \operatorname{ad}(X) Y_{j}, Y_{j}\right\rangle \\
& =\sum_{j=1}^{n}\left\langle\operatorname{ad}(X) Y_{j}, \operatorname{Ad}(x) Y_{j}\right\rangle \\
& =-\sum_{j=1}^{n}\left\langle Y_{j}, \operatorname{ad}(X) \operatorname{Ad}(x) Y_{j}\right\rangle \\
& =-\sum_{j=1}^{n}\left\langle\operatorname{ad}(X) \operatorname{Ad}(x) Y_{j}, Y_{j}\right\rangle \\
& =-\operatorname{tr}\{\operatorname{ad}(X) \cdot \operatorname{Ad}(x)\} \\
& =-\operatorname{tr}\{\operatorname{Ad}(x) \cdot \operatorname{ad}(X)\} \\
& =0
\end{aligned}
$$

pois $x \in E_{W}$. Como $X \in \mathfrak{g}$ é arbitrário, concluímos que $x^{-1} \in E_{W}$.

Inspirados na proposição acima, poderíamos imaginar que $E_{W}$ é um subgrupo de $G$. De fato, mostraremos a seguir que se esta conjectura for verdadeira então $E_{W}$ é discreto.

Proposição 5.5. Suponha que $G$ é semi-simples e que $E_{W}$ é um subgrupo de $G$. Então $E_{W}$ é um subconjunto discreto de $G$. Em particular, a identidade e é um ponto isolado de $E_{W}$.

Demonstração. Primeiramente, observe que $E_{W}$ é um subconjunto fechado de $G$. Se além disso ele for um subgrupo de $G$ então pelo Teorema do Subgrupo Fechado ${ }^{1}$ temos que $E_{W}$ é um subgrupo de Lie de $G$. Denotemos por $\mathfrak{h} \subset \mathfrak{g}$ sua álgebra de Lie.

\footnotetext{
${ }^{1}$ [Lee03], Theorem 20.10, página 526.
} 
Fixemos $X \in \mathfrak{h}$. Para cada $Y \in \mathfrak{g}$ defina $f_{Y}: \mathbb{R} \rightarrow \mathbb{R}$ por

$$
f_{Y}(t) \doteq \operatorname{tr}\left\{\operatorname{Ad}\left(\mathbf{e}^{t X}\right) \cdot \operatorname{ad}(Y)\right\}
$$

para cada $t \in \mathbb{R}$. Como $X \in \mathfrak{h}$ temos que $\mathbf{e}^{t X} \in E_{W}$ para todo $t \in \mathbb{R}$ de modo que segundo a Proposição 5.3 temos

$$
f_{Y}(t)=0 \text { para todo } t \in \mathbb{R} \text {. }
$$

Derivando temos

$$
\begin{aligned}
f_{Y}^{\prime}(t) & =\frac{d}{d t} \operatorname{tr}\left\{\operatorname{Ad}\left(\mathbf{e}^{t X}\right) \cdot \operatorname{ad}(Y)\right\} \\
& =\operatorname{tr}\left\{\frac{d}{d t} \operatorname{Ad}\left(\mathbf{e}^{t X}\right) \cdot \operatorname{ad}(Y)\right\} \\
& =\operatorname{tr}\left\{\operatorname{Ad}\left(\mathbf{e}^{t X}\right) \cdot \operatorname{ad}\left(d L_{\mathbf{e}^{-t X}} \frac{d}{d t} \mathbf{e}^{t X}\right) \cdot \operatorname{ad}(Y)\right\} \\
& =\operatorname{tr}\left\{\operatorname{Ad}\left(\mathbf{e}^{t X}\right) \cdot \operatorname{ad}\left(d L_{\mathbf{e}^{-t X} X}\left(\mathbf{e}^{t X}\right)\right) \cdot \operatorname{ad}(Y)\right\} \\
& =\operatorname{tr}\left\{\operatorname{Ad}\left(\mathbf{e}^{t X}\right) \cdot \operatorname{ad}(X) \cdot \operatorname{ad}(Y)\right\}
\end{aligned}
$$

que é igual a 0 para todo $t \in \mathbb{R}$. Em particular para $t=0$ temos que

$$
\begin{aligned}
f_{Y}^{\prime}(0) & =\operatorname{tr}\{\operatorname{Ad}(e) \cdot \operatorname{ad}(X) \cdot \operatorname{ad}(Y)\} \\
& =\operatorname{tr}\{\operatorname{ad}(X) \cdot \operatorname{ad}(Y)\}
\end{aligned}
$$

é igual a 0 , ou seja

$$
B(X, Y)=0
$$

onde $B$ é a forma de Killing de $\mathfrak{g}$ introduzida na Seção 2.1. Como $Y \in \mathfrak{g}$ é arbitrário e $G$ por hipótese é semi-simples concluímos que $X=0$.

Como $X \in \mathfrak{h}$ é arbitrário temos $\mathfrak{h}=\{0\}$ e, portanto, $E_{W}$ é um subgrupo discreto de $G$.

A conclusão acima é até mais forte. Como $E_{W}$ é fechado e $G$ é por hipótese compacto temos que $E_{W}$ é compacto. Sendo discreto, $E_{W}$ é finito! Infelizmente, não conseguimos demonstrar que $E_{W}$ é um subgrupo de $G$ (e honestamente não acreditamos que isso seja verdade), de modo que teremos que empregar outra técnica para demonstrar que $e$ é um ponto isolado de $E_{W}$ - e, mais que isso, é um atrator local de $W-$, a qual introduzimos a seguir.

Definição 5.6. Sejam $M$ uma variedade e $f: M \rightarrow \mathbb{R}$ uma função suave. Dizemos que $x \in M$ é um ponto crítico não-degenerado de $f$ se

- $x$ é ponto crítico de $f$ i.e. $d f_{x}=0$ e 
- existe uma vizinhança coordenada $(U, \phi)$ de $x$ tal que a matriz Hessiana de $f \circ \phi^{-1}$

$$
\left[\frac{\partial^{2}\left(f \circ \phi^{-1}\right)}{\partial x_{j} \partial x_{k}}\right]_{1 \leq j, k \leq n}
$$

é não-singular no ponto $\phi(x)$.

É possível mostrar que embora a matriz Hessiana dependa da vizinhança coordenada escolhida a condição de não-degenerescência é invariante: se a matriz Hessiana de $f$ é não-singular em algum sistema de coordenadas local de um dado ponto então o é em qualquer sistema de coordenadas local do ponto em questão. É uma consequência do Lema de Morse ${ }^{2}$ que todo ponto crítico nãodegenerado de uma função suave é isolado, isto é, possui uma vizinhança que não contém outros pontos críticos. Para mais detalhes sobre esses assuntos consultar [Mil63], Parte I, parágrafo 2.

Procedemos provando que todo $x \in Z(G)$ é um ponto crítico não-degenerado de $V$. Com tal finalidade introduzimos uma família especial de sistemas de coordenadas em $G$, às vezes chamados na literatura de coordenadas de segunda espécie (coordinates of second kind).

Lema 5.7. Sejam $x \in G, Y_{1}, \ldots, Y_{n} \in \mathfrak{g}$ e $\Phi: \mathbb{R}^{n} \rightarrow G$ dada por

$$
\Phi\left(t_{1}, \ldots, t_{n}\right) \doteq x \cdot \mathbf{e}^{t_{1} Y_{1}} \cdot \ldots \cdot \mathbf{e}^{t_{n} Y_{n}}
$$

para cada $\left(t_{1}, \ldots, t_{n}\right) \in \mathbb{R}^{n}$. Então

$$
d \Phi\left(\left.\frac{\partial}{\partial t_{j}}\right|_{\left(\bar{t}_{1}, \ldots, \bar{t}_{n}\right)=(0, \ldots, 0)}\right)=Y_{j}(x)
$$

para cada $j=1, \ldots, n$. Em particular, se $Y_{1}, \ldots, Y_{n}$ formam uma base de $\mathfrak{g}$ então a aplicação $\Phi$ definida acima é um difeomorfismo local em uma vizinhança de $(0, \ldots, 0) \in \mathbb{R}^{n}$.

Demonstração. Dada $f \in C^{\infty}(G)$ temos, denotando

$$
\begin{aligned}
& \overline{\mathbf{t}} \doteq\left(\bar{t}_{1}, \ldots, \bar{t}_{n}\right) \\
& \mathbf{0} \doteq(0, \ldots, 0)
\end{aligned}
$$

\footnotetext{
${ }^{2}$ [Mil63], Lemma 2.2 e Corollary 2.3, páginas 6-8.
} 
elementos de $\mathbb{R}^{n}$, que

$$
\begin{aligned}
d \Phi\left(\left.\frac{\partial}{\partial t_{j}}\right|_{\overline{\mathbf{t}}=\mathbf{0}}\right)(f) & =\left.\frac{\partial}{\partial t_{j}}\right|_{\overline{\mathbf{t}}=\mathbf{0}} f \circ \Phi \\
& =\left.\frac{\partial}{\partial t_{j}}\right|_{\overline{\mathbf{t}}=\mathbf{0}} f\left(x \cdot \mathbf{e}^{t_{1} Y_{1}} \cdot \ldots \cdot \mathbf{e}^{t_{j-1} Y_{j-1}} \cdot \mathbf{e}^{t_{j} Y_{j}} \cdot \mathbf{e}^{t_{j+1} Y_{j+1}} \cdot \ldots \cdot \mathbf{e}^{t_{n} Y_{n}}\right) \\
& =\left.\frac{d}{d s}\right|_{s=0} f\left(x \cdot \mathbf{e}^{0 Y_{1}} \cdot \ldots \cdot \mathbf{e}^{0 Y_{j-1}} \cdot \mathbf{e}^{s Y_{j}} \cdot \mathbf{e}^{0 Y_{j+1}} \cdot \ldots \cdot \mathbf{e}^{0 Y_{n}}\right) \\
& =\left.\frac{d}{d s}\right|_{s=0} f\left(x \cdot \mathbf{e}^{s Y_{j}}\right) \\
& =\left(\left.\frac{d}{d s}\right|_{s=0} x \cdot \mathbf{e}^{s Y_{j}}\right)(f) \\
& =Y_{j}(x)(f) .
\end{aligned}
$$

Como $f \in C^{\infty}(G)$ é arbitrária concluímos que

$$
d \Phi\left(\left.\frac{\partial}{\partial t_{j}}\right|_{\overline{\mathbf{t}=\mathbf{0}}}\right)=Y_{j}(x) .
$$

Agora, se $Y_{1}, \ldots, Y_{n}$ formam uma base de $\mathfrak{g}$ então $Y_{1}(x), \ldots, Y_{n}(x)$ formam uma base de $T_{x} G$. A igualdade anterior implica, nesse caso, que $d \Phi_{\mathbf{0}}$ é um isomorfismo linear, de modo que a conclusão segue do Teorema da Função Inversa.

Proposição 5.8. Se $G$ é semi-simples então todo $x \in Z(G)$ é um ponto crítico não-degenerado da função "tipo Lyapunov" $V$. Em particular todo $x \in Z(G)$ é ponto isolado de $E_{W}$ e, portanto, $Z(G)$ é aberto em $E_{W}$.

Demonstração. Primeiramente recordemos que $Z(G) \subset E_{W}$, segundo o Corolário 4.9, de modo que $x$ é um ponto de equilíbrio de $W$. A Proposição 5.3 implica, então, que $x$ é ponto crítico de $V$, visto que estamos supondo que a trajetória de referência $\left(\mathbf{x}_{\mathbf{r}}, u_{1}^{r}, \ldots, u_{m}^{r}\right)$ em questão é regular.

A fim de provar a não-degenerescência, vamos calcular a matriz Hessiana de $V$ em um sistema de coordenadas especial ao redor de $x$ que construímos na sequência. Já vimos nos comentários que sucedem o Teorema 2.17 que se $G$ semi-simples então $-B$ é um produto interno em $\mathfrak{g}$, onde $B$ é a forma de Killing de $\mathfrak{g}$. Assim sendo, considere $Y_{1}, \ldots, Y_{n} \in \mathfrak{g}$ uma base ortonormal de $\mathfrak{g}$ com relação a esse produto interno e defina $\Phi: \mathbb{R}^{n} \rightarrow G$ por

$$
\Phi\left(t_{1}, \ldots, t_{n}\right) \doteq x \cdot \mathbf{e}^{t_{1} Y_{1}} \cdot \ldots \cdot \mathbf{e}^{t_{n} Y_{n}}
$$

para cada $\left(t_{1}, \ldots, t_{n}\right) \in \mathbb{R}^{n}$ como no lema anterior, o qual nos diz que existe uma vizinhança aberta $U \subset G$ de $x$ tal que $\left.\phi \doteq \Phi^{-1}\right|_{U}$ é um sistema de coordenadas de $G$ i.e. $(U, \phi)$ é uma vizinhança coordenada de $x$. 
Vamos computar as entradas da matriz Hessiana de $V$ neste sistema de coordenadas. Observe que

$$
\begin{aligned}
\left(V \circ \phi^{-1}\right)\left(t_{1}, \ldots, t_{n}\right) & =V\left(\Phi\left(t_{1}, \ldots, t_{n}\right)\right) \\
& =\operatorname{tr}\left\{\operatorname{Ad}\left(x \cdot \mathbf{e}^{t_{1} Y_{1}} \cdot \ldots \cdot \mathbf{e}^{t_{n} Y_{n}}\right)\right\} \\
& =\operatorname{tr}\left\{\operatorname{Ad}(x) \cdot \operatorname{Ad}\left(\mathbf{e}^{t_{1} Y_{1}}\right) \cdot \ldots \cdot \operatorname{Ad}\left(\mathbf{e}^{t_{n} Y_{n}}\right)\right\} \\
& =\operatorname{tr}\left\{\operatorname{Ad}(x) \cdot \mathbf{e}^{\operatorname{ad}\left(t_{1} Y_{1}\right)} \cdot \ldots \cdot \mathbf{e}^{\operatorname{ad}\left(t_{n} Y_{n}\right)}\right\} \\
& =\operatorname{tr}\left\{\mathbf{e}^{t_{1} \operatorname{ad}\left(Y_{1}\right)} \cdot \ldots \cdot \mathbf{e}^{t_{n} \operatorname{ad}\left(Y_{n}\right)}\right\}
\end{aligned}
$$

onde usamos que $\operatorname{Ad}(x)=\mathrm{id}_{\mathfrak{g}}$ (pois, conforme (2.9) todo ponto de $Z(G)$ está no núcleo de $\operatorname{Ad}$ ) e a identidade (2.7). Uma conta simples mostra então que no ponto $\phi(x)=(0, \ldots, 0)$ temos

$$
\begin{aligned}
\frac{\partial^{2}\left(V \circ \phi^{-1}\right)}{\partial t_{j} \partial t_{k}} & =\operatorname{tr}\left\{\operatorname{ad}\left(Y_{j}\right) \cdot \operatorname{ad}\left(Y_{k}\right)\right\} \\
& =B\left(Y_{j}, Y_{k}\right) \\
& =-\delta_{j k}
\end{aligned}
$$

para quaisquer $1 \leq j, k \leq n$. Isto prova que no ponto $\phi(x)$ temos

$$
\operatorname{det}\left\{\left[\frac{\partial^{2}\left(V \circ \phi^{-1}\right)}{\partial t_{j} \partial t_{k}}\right]_{1 \leq j, k \leq n}\right\}=(-1)^{n}
$$

e, portanto, $x$ é um ponto crítico não-degenerado de $V$.

Como todo ponto crítico não-degenerado é isolado, a conclusão acima implica que todo ponto de $Z(G)$ possui uma vizinhança em $G$ cuja intersecção com $E_{W}$ é trivial e, portanto, contida em $Z(G)$. Logo $Z(G)$ é aberto em $E_{W}$.

Os próximos dois lemas caracterizam o centro de $G$ em termos de $V$ e não dependem da hipótese de $G$ ser semi-simples. Para demonstrá-los precisamos empregar o processo de complexificação de espaços vetoriais: nos argumentos seguir, $\mathfrak{g}_{\mathbb{C}}$ denota a complexificação de $\mathfrak{g}$ - a qual é um espaço vetorial sobre $\mathbb{C}$ com dimensão complexa igual a $n \doteq \operatorname{dim} \mathfrak{g}-\mathrm{e}$

$$
\operatorname{Ad}_{\mathbb{C}}(x): \mathfrak{g}_{\mathbb{C}} \rightarrow \mathfrak{g}_{\mathbb{C}}
$$

a complexificação do operador $\operatorname{Ad}(x)$. É um fato bem conhecido que

$$
\operatorname{tr} \operatorname{Ad}(x)=\operatorname{trAd}_{\mathbb{C}}(x)=\sum_{j=1}^{n} \lambda_{j}(x)
$$

onde $\lambda_{1}(x), \ldots, \lambda_{n}(x) \in \mathbb{C}$ são os autovalores de $\operatorname{Ad}_{\mathbb{C}}(x)$, repetidos segundo suas multiplicidades 
algébricas.

Considere agora $\langle\cdot, \cdot\rangle$ um produto interno Ad-invariante em $\mathfrak{g}$ cuja existência é garantida pela Proposição 2.15. Não é difícil mostrar que $\langle\cdot, \cdot\rangle$ possui uma única extensão sesquilinear $\langle\cdot, \cdot\rangle_{\mathbb{C}}$ a $\mathfrak{g}_{\mathbb{C}}$, a qual é um produto interno hermitiano em $\mathfrak{g}_{\mathbb{C}}$ que satisfaz a propriedade de ser invariante por $\operatorname{Ad}_{\mathbb{C}}(x)$ i.e. este é um operador unitário com relação a $\langle\cdot, \cdot\rangle_{\mathbb{C}}$. Isto implica, segundo um fato bem conhecido de Álgebra Linear, que $\operatorname{Ad}_{\mathbb{C}}(x)$ é diagonalizável e que todos os seus autovalores têm módulo igual a 1 ou seja

$$
\left|\lambda_{j}(x)\right|=1 \text { para } j=1, \ldots, n .
$$

A digressão acima implica

Lema 5.9. Se $x \in G$ então

$$
V(x) \leq \operatorname{dim} \mathfrak{g} .
$$

Em particular, todo $x \in Z(G)$ é ponto de máximo global de $V$.

Demonstração. É só observar que

$$
\begin{aligned}
\left|\operatorname{trAd}_{\mathbb{C}}(x)\right| & =\left|\sum_{j=1}^{n} \lambda_{j}(x)\right| \\
& \leq \sum_{j=1}^{n}\left|\lambda_{j}(x)\right| \\
& \leq \sum_{j=1}^{n} 1 \\
& =n
\end{aligned}
$$

mas, por definição,

$$
V(x)=\operatorname{tr} \operatorname{Ad}(x)=\operatorname{trAd}_{\mathbb{C}}(x)
$$

de modo que

$$
\begin{aligned}
V(x) & \leq|V(x)| \\
& =\left|\operatorname{trAd}_{\mathbb{C}}(x)\right| \\
& \leq n .
\end{aligned}
$$

Em particular, se $x \in Z(G)$ então $\operatorname{Ad}(x)=\operatorname{id}_{\mathfrak{g}}$ (veja (2.9)) de modo que

$$
V(x)=n
$$


e pelo argumento anterior

$$
V(y) \leq n=V(x)
$$

para todo $y \in G$. Ou seja, $x$ é um ponto de máximo global de $V$.

Lema 5.10. Dado $x \in G$ temos que

$$
x \in Z(G) \Leftrightarrow V(x)=\operatorname{dim} \mathfrak{g} .
$$

Demonstração. Já sabemos que se $x \in Z(G)$ então $V(x)=n$. Reciprocamente, suponha que $V(x)=n$. Então

$$
n=\sum_{j=1}^{n} \lambda_{j}(x)=\sum_{j=1}^{n} \Re \lambda_{j}(x)+i \sum_{j=1}^{n} \Im \lambda_{j}(x)
$$

o que implica que

$$
\begin{aligned}
& \sum_{j=1}^{n} \Im \lambda_{j}(x)=0 \\
& \sum_{j=1}^{n} \Re \lambda_{j}(x)=n .
\end{aligned}
$$

Observe ainda que

$$
\Re \lambda_{j}(x) \leq\left|\Re \lambda_{j}(x)\right| \leq\left|\lambda_{j}(x)\right|=1
$$

para cada $j=1, \ldots, n$. Vamos provar que $\Re \lambda_{j}(x)=1$ para todo $j=1, \ldots, n$. Com efeito, se $\Re \lambda_{k}(x)<1$ para algum $k \in\{1, \ldots, n\}$ então

$$
\sum_{j=1}^{n} \Re \lambda_{j}(x)=\Re \lambda_{k}(x)+\sum_{j \neq k} \Re \lambda_{j}(x)<n
$$

o que contradiz nossa conclusão anterior. Isto prova que $\Re \lambda_{j}(x)=1$ para todo $j=1, \ldots, n$.

Além disso $\left|\lambda_{j}(x)\right|=1$ para todo $j=1, \ldots, n$ : como pelo argumento acima $\Re \lambda_{j}(x)=1$ temos que

$$
\Im \lambda_{j}(x)=0 \text { para todo } j=1, \ldots, n \text {. }
$$

Daí $\lambda_{j}(x)=\Re \lambda_{j}(x)=1$ para todo $j=1, \ldots, n$. Como $\lambda_{1}(x), \ldots, \lambda_{n}(x)$ são os autovalores de $\operatorname{Ad}_{\mathbb{C}}(x)$ concluímos que

$$
\operatorname{Ad}_{\mathbb{C}}(x)=\operatorname{id}_{\mathfrak{g}_{\mathbb{C}}}
$$


o que por sua vez implica que $\operatorname{Ad}(x)=\mathrm{id}_{\mathfrak{g}}$, ou seja, $x \in Z(G)$.

Já podemos demonstrar o resultado principal do presente trabalho. Recordemos que $W$ é o campo auxiliar associado a uma trajetória de referência periódica regular.

Teorema 5.11. Se $G$ é semi-simples então todo $w \in Z(G)$ é um atrator local de $W$.

Demonstração. Como $w \in Z(G)$ a Proposição 5.8 implica que existe uma vizinhança aberta $U \subset G$ de $w$ que não contém outros pontos críticos de $V$ ou seja

$$
U \cap E_{W}=\{w\}
$$

Seja $U^{\prime} \subset G$ um aberto conexo que contém $w$ e cujo fecho, necessariamente compacto, está contido em $U$. Em particular, sua fronteira $\partial U^{\prime}$, também compacta, está contida em $U$. Definimos

$$
M \doteq \max _{x \in \partial U^{\prime}} V(x)
$$

De acordo com os lemas anteriores temos que $M<n$ pois $\partial U^{\prime} \cap Z(G)=\emptyset$ : o único ponto em $U \cap Z(G)$ é $w$, o qual pertence a $U^{\prime}$ (e portanto não está na fronteira).

Como $V(w)=n$ e $V$ é contínua existe $U^{\prime \prime} \subset U^{\prime}$ vizinhança aberta de $e$ tal que

$$
V(x)>M \text { para todo } x \in U^{\prime \prime}
$$

Seja agora $\left(t_{0}, w_{0}\right) \in \mathbb{R} \times U^{\prime \prime}$ e $\mathbf{w}: \mathbb{R} \rightarrow G$ a única curva integral de $W$ que satisfaz $\mathbf{w}\left(t_{0}\right)=w_{0}$. Como $V \circ \mathbf{w}$ é não decrescente (pois $V$ é não decrescente ao longo de $W$, conforme já observado na Seção 4.2) temos, para todo $t \geq t_{0}$, que

$$
V(\mathbf{w}(t)) \geq V\left(\mathbf{w}\left(t_{0}\right)\right)=V\left(w_{0}\right)>M
$$

pois $w_{0} \in U^{\prime \prime}$ por hipótese. Em particular $\mathbf{w}(t) \notin \partial U^{\prime}$ para todo $t \geq t_{0}$, de modo que pela continuidade de $\mathbf{w}$ temos que

$$
\mathbf{w}(t) \in U^{\prime} \text { para todo } t \geq t_{0}
$$

Por fim, provemos que

$$
\lim _{t \rightarrow \infty} \mathbf{w}(t)=w
$$

De fato vamos provar que toda sequência crescente ilimitada superiormente $\left(t_{n}\right)_{n \in \mathbb{N}}$ possui uma subsequência $\left(t_{n_{k}}\right)_{k \in \mathbb{N}}$ tal que

$$
\lim _{k \rightarrow \infty} \mathbf{w}\left(t_{n_{k}}\right)=w
$$


Com efeito, se $\left(t_{n}\right)_{n \in \mathbb{N}}$ é uma tal sequência - a qual, podemos supor, satisfaz $t_{n} \geq t_{0}$ para todo $n \in \mathbb{N}-$ temos que

$$
\mathbf{w}\left(t_{n}\right) \in U^{\prime} \text { para todo } n \in \mathbb{N} \text {. }
$$

Além disso, $U^{\prime}$ possui fecho compacto contido em $U$, de modo que necessariamente existem uma subsequência $\left(t_{n_{k}}\right)_{k \in \mathbb{N}}$ de $\left(t_{n}\right)_{n \in \mathbb{N}}$ e $w_{1} \in U$ tal que

$$
\lim _{k \rightarrow \infty} \mathbf{w}\left(t_{n_{k}}\right)=w_{1}
$$

Observe que $\left(t_{n_{k}}\right)_{k \in \mathbb{N}}$ também deve necessariamente ser uma sequência crescente e ilimitada superiormente, o que implica que $w_{1}$ pertence a $\Omega_{W}\left(t_{0}, w_{0}\right)$ e, portanto, a $E_{W}$. Contudo por definição de $U$ temos

$$
U \cap E_{W}=\{w\}
$$

o que prova que $w_{1}=w$. Concluímos que

$$
\lim _{k \rightarrow \infty} \mathbf{w}\left(t_{n_{k}}\right)=w
$$

conforme prometêramos.

Tomando $w=e$ no teorema acima concluímos em particular que $e$ é um atrator local de $W$.

\subsection{Comentários e referências}

O conceito de trajetória regular no sentido da Definição 5.1 é original e foi introduzido precisamente para garantir a validade da Proposição 5.3 a partir do Teorema 4.7 sem criar complicações adicionais. O ponto importante, contudo, é ter condições suficientes para garantir que todo ponto $\Omega$-limite do campo auxiliar $W$ seja ponto crítico de $V$ (a recíproca é sempre verdadeira pois todo ponto crítico de $V$ é trivialmente um ponto de equilíbrio de $W$ e, portanto, um ponto $\Omega$-limite). A existência de trajetórias de referência $T$-periódicas com condições iniciais arbitrárias é demonstrada no Apêndice A, onde construímos uma trajetória desse tipo que é regular sempre que o sistema (3.1) satisfaz algumas hipóteses adicionais. Também no presente capítulo nossa abordagem tem a vantagem de obter resultados análogos àqueles apresentados em [Sil09] sem recorrer a uma descrição explícita da trajetória de referência: a identificação da regularidade da trajetória como um elemento chave do problema foi decisiva nesse desenvolvimento. Sobre este assunto, observamos por fim que nosso conceito de regularidade não possui ligação direta com o conceito de sistema T-regular introduzido por [Sil09], Definição 4.2, página 85.

Embora com um enunciado bastante distinto, nosso Teorema 5.11 é análogo aos resultados apresentados em [Sil09], Lema 4.18 e Teorema 4.20, páginas 103-108 (note que $G=\operatorname{SU}(n)$ é 
semi-simples $^{3}$ se $n \geq 2$ ). A técnica que empregamos para demonstrá-lo - introduzir coordenadas de segunda espécie para analisar a degenerescência dos pontos críticos -, contudo, é radicalmente diferente.

A seguir, descrevemos informalmente um projeto que desenvolvemos na tentativa de generalizar a técnica empregada por [Sil09], páginas 103-108, para resolver o problema acima. Os conceitos envolvidos podem ser consultados em [Kna02] ou [Sep07].

1. O objetivo do projeto é provar que $V\left(E_{W}\right)$ é um conjunto finito: aparentemente não é difícil provar, a partir daí, que os pontos de $Z(G)$ são equilíbrios atratores de $W$, mas é muito mais forte que isso, o que torna este projeto mais ambicioso do que aquele desenvolvido na seção anterior.

2. Primeiramente, é possível provar que, sem perda de generalidade, podemos restringir esse problema a qualquer toro maximal de $G$ : se $\mathbb{T} \subset G$ é um toro maximal então

$$
V\left(E_{W}\right)=V\left(E_{W} \cap \mathbb{T}\right)
$$

Isto é uma consequência da Proposição 5.4.

3. Em seguida, fixado um toro maximal $\mathbb{T} \subset G$, empregamos a Decomposição em Espaços de Raízes de $\mathfrak{g}_{\mathbb{C}}$ com relação à álgebra de Cartan $\mathfrak{t}$ de $\mathbb{T}$, onde $\mathfrak{g}_{\mathbb{C}}$ é a complexificação de $\mathfrak{g}$. Observamos que se $x \in \mathbb{T}$ então $\operatorname{Ad}(x)$ age trivialmente em $\mathfrak{t}$ e, portanto, sua complexificação $\operatorname{Ad}_{\mathbb{C}}(x)$ age trivialmente em $\mathfrak{t}_{\mathbb{C}}$. Juntamente com o fato de que os autoespaços da Decomposição são unidimensionais, isto implica que estes também são autoespaços de $\operatorname{Ad}_{\mathbb{C}}(x)$, e portanto a Decomposição em Espaços de Raízes diagonaliza $\operatorname{Ad}_{\mathbb{C}}(x)$.

4. O argumento anterior permite escrever

$$
V(x)=\operatorname{tr} \operatorname{Ad}(x)=\operatorname{trAd}_{\mathbb{C}}(x)
$$

convenientemente como soma de autovalores de $\operatorname{Ad}_{\mathbb{C}}(x)$.

5. A descrição (5.1) é facilmente estendida para

$$
E_{W}=\left\{x \in G ; \operatorname{tr}\left\{\operatorname{Ad}_{\mathbb{C}}(x) \cdot \operatorname{ad}_{\mathbb{C}}(Z)\right\}=0 \text { para todo } Z \in \mathfrak{g}_{\mathbb{C}}\right\}
$$

onde $\operatorname{ad}_{\mathbb{C}}$ é a representação adjunta da álgebra de Lie complexa $\mathfrak{g}_{\mathbb{C}}$.

6. Especializando a fórmula acima em um vetor $Z \in \mathfrak{t}_{\mathbb{C}}$ e escolhendo uma base de $\mathfrak{g}_{\mathbb{C}}$ compatível com a Decomposição em Espaços de Raízes para computar

$$
\operatorname{tr}\left\{\operatorname{Ad}_{\mathbb{C}}(x) \cdot \operatorname{ad}_{\mathbb{C}}(Z)\right\}
$$

\footnotetext{
${ }^{3}$ [Kna02], página 113.
} 
obtemos um sistema linear homogêneo para as partes imaginárias dos autovalores de $\operatorname{Ad}_{\mathbb{C}}(x)$ quando $x \in E_{W} \cap \mathbb{T}$.

7. Infelizmente o sistema linear descrito acima é subdeterminado, e não nos fornece muita informação a respeito dos autovalores de $\operatorname{Ad}_{\mathbb{C}}(x)$ quando $x \in E_{W} \cap \mathbb{T}$.

Infelizmente, devido ao último passo descrito acima, não conseguimos concluir este projeto. Acreditamos, porém, que seja possível remediá-lo (talvez com algum conhecimento adicional da Decomposição em Espaços de Raízes) e que seja possível extrair dele mais informações a respeito dos valores críticos do caractere da representação adjunta de $G$.

Os passos finais da demonstração do Teorema 5.11 foram inspirados na demonstração do Critério de Lyapunov, conforme apresentado em [Sot79], Capítulo VIII, Seção 2, Teorema 3, página 272. Conforme observado pelo Prof. Hector Bessa Silveira em comunicação pessoal, a demonstração deste teorema prova muito mais do que foi enunciado: que os pontos de $Z(G)$ são equilíbrios uniformemente estáveis do campo auxiliar. 


\section{Apêndice A}

\section{Construção de uma trajetória de referência regular}

O objetivo deste apêndice é demonstrar que se a família $\Gamma$ é regular (Definição A.5) então o sistema (3.1) possui trajetórias de referência $T$-periódicas regulares passando por pontos arbitrários. Mais que meramente demonstrar a existência de uma tal trajetória, sob tais hipótese nós construiremos uma explicitamente!

Neste apêndice $G$ é um grupo de Lie compacto e conexo, com álgebra de Lie $\mathfrak{g}$, e $X_{1}, \ldots, X_{m} \in \mathfrak{g}$ são campos invariantes à esquerda fixados. Ademais, não supomos que

$$
\Gamma \doteq \operatorname{span}\left\{X_{1}, \ldots, X_{m}\right\}
$$

é Lie-determinada.

\section{A.1 Construindo uma trajetória de referência periódica}

Fixemos $x_{\infty} \in \mathcal{O}_{\Gamma}$ e $T>0$. Para cada $j=1, \ldots, m$ denotamos, para cada $p \in \mathbb{Z}$,

$$
\begin{aligned}
J_{j}^{p} & \doteq\left(\frac{(j-1) T}{m}+p T, \frac{j T}{m}+p T\right) \\
& =J_{j}^{0}+p T .
\end{aligned}
$$

Observe que $J_{j}^{p} \subset(p T,(p+1) T)=(0, T)+p T$ para todo $p \in \mathbb{Z}$ e que

$$
J_{j}^{p} \cap J_{k}^{q} \neq \emptyset \Leftrightarrow j=k, p=q
$$

Considere $\chi_{j} \in C_{c}^{\infty}\left(J_{j}^{0}\right)$ com as seguintes propriedades:

1. existe um intervalo aberto $I_{j} \subset J_{j}^{0}$ tal que

$$
\chi_{j}(t)=1 \text { para todo } t \in I_{j}
$$

2. $\int_{0}^{T} \chi_{j}(t) d t=0$.

Funções $\chi_{j}$ com as propriedades acima podem ser construídas a partir de funções de corte através de translações e reflexões. Por exemplo, se $\phi \in C_{c}^{\infty}((-1,1))$ é tal que $\phi(t)=1$ para $t \in(-1 / 2,1 / 2)$ 
então

$$
\chi_{j}(t) \doteq \begin{cases}\phi\left(\frac{4 m}{T} t-4 j+3\right), & \text { se } t \in\left(\frac{(j-1) T}{m}, \frac{j T}{m}-\frac{T}{2 m}\right) \\ -\phi\left(\frac{4 m}{T} t-4 j+1\right), & \text { se } t \in\left(\frac{j T}{m}-\frac{T}{2 m}, \frac{j T}{m}\right) \\ 0, & \text { caso contrário }\end{cases}
$$

define, para cada $j=1, \ldots, m$, uma função $\chi_{j}: \mathbb{R} \rightarrow \mathbb{R}$ com as propriedades pedidas acima, tomando, por exemplo,

$$
I_{j} \doteq\left(\frac{j T}{m}-\frac{7 T}{8 m}, \frac{j T}{m}-\frac{5 T}{8 m}\right)
$$

(As contas acima são muito mais simples do que parecem: apenas aplicamos transformações afins

$$
\begin{aligned}
& (-1,1) \rightarrow\left(\frac{(j-1) T}{m}, \frac{j T}{m}-\frac{T}{2 m}\right) \\
& (-1,1) \rightarrow\left(\frac{j T}{m}-\frac{T}{2 m}, \frac{j T}{m}\right)
\end{aligned}
$$

convenientes e carregamos as funções $\phi$ e $-\phi$, respectivamente, colando-as no ponto médio

$$
\frac{j T}{m}-\frac{T}{2 m}=\frac{1}{2}\left(\frac{(j-1) T}{m}+\frac{j T}{m}\right)
$$

a fim de anular a integral de $\chi_{j}$ em $J_{j}^{0}$. O intervalo $I_{j}$ é, então, simplesmente a imagem do intervalo $(-1 / 2,1 / 2)$ pela primeira transformação afim.)

Considere agora $u_{j}^{r}: \mathbb{R} \rightarrow \mathbb{R}$ função $T$-periódica tal que

$$
u_{j}^{r}(t)=\chi_{j}(t) \text { para todo } t \in[0, T]
$$

Estas duas propriedades determinam unicamente $u_{j}^{r}$ (observe que $\chi_{j}(0)=0=\chi_{j}(T)$ ), a qual é necessariamente suave. Ou seja, $u_{j}^{r}$ é a extensão $T$-periódica de $\left.\chi_{j}\right|_{[0, T]}$.

Listamos a seguir as propriedades fundamentais dos controles $u_{j}^{r}$ que construímos acima. Na verdade, esses controles foram construídos de forma a valerem as propriedades enunciadas na sequência.

Proposição A.1. supp $u_{j}^{r} \subset \bigcup_{p \in \mathbb{Z}} J_{j}^{p}$.

Demonstração. Suponha que $t \notin \bigcup_{p \in \mathbb{Z}} J_{j}^{p}$ e seja $q \in \mathbb{Z}$ tal que $t-q T \in[0, T]$. Por hipótese, $t-q T \notin J_{j}^{0}$ e, como

$$
\operatorname{supp} \chi_{j} \subset J_{j}^{0}
$$


então $t-q T \notin \operatorname{supp} \chi_{j}$, de modo que existe uma vizinhança $U$ de $t-q T$ tal que

$$
\chi_{j}(s)=0 \text { para todo } s \in U \text {. }
$$

Daí $U+q T$ é uma vizinhança de $t$ onde $u_{j}^{r}$ se anula, e portanto $t \notin \operatorname{supp} u_{j}^{r}$.

Proposição A.2. Suponha que $t \in \mathbb{R}$ é tal que existe $j \in\{1, \ldots, m\}$ tal que

$$
u_{j}^{r}(t) \neq 0
$$

Então $u_{k}^{r}(t)=0$ para todo $k \neq j$.

Demonstração. Basta observar que se $u_{j}^{r}(t) \neq 0$ então

$$
t \in \operatorname{supp} u_{j}^{r}
$$

de modo que, pela proposição anterior, existe $p \in \mathbb{Z}$ tal que $t \in J_{j}^{p}$. Analogamente, se $u_{k}^{r}(t) \neq 0$ existe $q \in \mathbb{Z}$ tal que $t \in J_{k}^{q}$. Mas então $t \in J_{j}^{p} \cap J_{k}^{q}$, o que implica que $j=k$.

Proposição A.3. Se $t \in I_{j}^{p} \doteq I_{j}+p T$ então

- $u_{j}^{r}(t)=1$,

- $u_{k}^{r}(t)=0$ se $k \neq j$ e

- todas as derivadas de $u_{k}^{r}$ se anulam em $t$ para $k=1, \ldots, m$ (inclusive $k=j$ ).

Demonstração. Se $t \in I_{j}^{p}$ então $t-p T \in I_{j}$ de modo que

$$
u_{j}^{r}(t)=\chi_{j}(t-p T)=1
$$

Do lema anterior, temos necessariamente que $u_{k}^{r}(t)=0$ se $k \neq j$. Como $I_{j}^{p}=I_{j}+p T$ é aberto, a última conclusão é imediata.

Defina agora $\xi_{j}: \mathbb{R} \rightarrow \mathbb{R}$ por

$$
\xi_{j}(t) \doteq \int_{0}^{t} u_{j}^{r}(s) d s
$$


de modo que $\xi_{j}$ é necessariamente suave. Observe que $\xi_{j}$ é também $T$-periódica pois

$$
\begin{aligned}
\xi_{j}(t+T) & =\int_{0}^{t+T} u_{j}^{r}(s) d s \\
& =\int_{0}^{T} u_{j}^{r}(s) d s+\int_{T}^{t+T} u_{j}^{r}(s) d s \\
& =\int_{0}^{T} \chi_{j}(s) d s+\int_{0}^{t} u_{j}^{r}(s-T) d s \\
& =\int_{0}^{t} u_{j}^{r}(s) d s \\
& =\xi_{j}(t)
\end{aligned}
$$

para todo $t \in \mathbb{R}$ (usamos que $u_{j}^{r}$ é $T$-periódica e que a integral de $\chi_{j}$ sobre $[0, T]$ é nula). Observe ainda que se $t-p T \in[0, T]$ então

$$
\begin{aligned}
\xi_{j}(t) & =\int_{0}^{t} u_{j}^{r}(s) d s \\
& =\int_{0}^{t} u_{j}^{r}(s-p T) d s \\
& =\int_{-p T}^{t-p T} u_{j}^{r}(s) d s \\
& =\int_{-p T}^{0} u_{j}^{r}(s) d s+\int_{0}^{t-p T} u_{j}^{r}(s) d s \\
& =\int_{0}^{t-p T} u_{j}^{r}(s) d s \\
& =\int_{0}^{t-p T} \chi_{j}(s) d s
\end{aligned}
$$

ou seja

$$
\xi_{j}(t)=\int_{0}^{t-p T} \chi_{j}(s) d s
$$

Proposição A.4. supp $\xi_{j} \subset \bigcup_{p \in \mathbb{Z}} J_{j}^{p}$.

Demonstração. Suponha que $t \notin \bigcup_{p \in \mathbb{Z}} J_{j}^{p}$ e seja $q \in \mathbb{Z}$ tal que $t-q T \in[0, T]$, de modo que

$$
t-q T \notin J_{j}^{0}=\left(\frac{(j-1) T}{m}, \frac{j T}{m}\right)
$$

e portanto há dois casos a serem analisados:

1. $t-q T \leq \frac{(j-1) T}{m} \mathrm{e}$

2. $t-q T \geq \frac{j T}{m}$. 
Recordando que supp $\chi_{j} \subset J_{j}^{0}$ temos que, em cada um dos casos, respectivamente, existe $\epsilon>0$ tal que

1. $\operatorname{supp} \chi_{j} \subset\left(t-q T+\epsilon, \frac{j T}{m}\right) \mathrm{e}$

2. $\operatorname{supp} \chi_{j} \subset\left(\frac{(j-1) T}{m}, t-q T-\epsilon\right)$.

Ainda, segundo a digressão anterior,

$$
\xi_{j}(t)=\int_{0}^{t-q T} \chi_{j}(s) d s
$$

Logo, no primeiro caso temos que $\chi_{j}(s)=0$ para todo $s \in[0, t-q T+\epsilon]$ e, portanto,

$$
\int_{0}^{r} \chi_{j}(s) d s=0
$$

para todo $r$ em uma vizinhança de $t-q T$. Isto, por sua vez, implica que $\xi_{j}$ se anula em uma vizinhança de $t$ e, portanto, $t \notin \operatorname{supp} \xi_{j}$.

No segundo caso temos que $\chi_{j}(s)=0$ para todo $s \in[t-q T-\epsilon, T]$ e, portanto,

$$
\int_{0}^{r} \chi_{j}(s) d s=\int_{0}^{T} \chi_{j}(s) d s=0
$$

para todo $r$ em uma vizinhança de $t-q T$. Isto, por sua vez, implica que $\xi_{j}$ se anula em uma vizinhança de $t$ e, portanto, $t \notin \operatorname{supp} \xi_{j}$.

Definimos agora $\mathbf{x}_{\mathbf{r}}: \mathbb{R} \rightarrow G$ da seguinte forma:

$$
\mathbf{x}_{\mathbf{r}}(t) \doteq \begin{cases}\mathbf{e}^{\xi_{j}(t) X_{j}}\left(x_{\infty}\right), & \text { se } t \in \bigcup_{p \in \mathbb{Z}} J_{j}^{p} \text { para } j=1, \ldots, m \\ x_{\infty}, & \text { caso contrário. }\end{cases}
$$

Observe que $\mathbf{x}_{\mathbf{r}}$ está bem definida em vista da observação (A.1). Ainda, a curva $\mathbf{x}_{\mathbf{r}}$ é suave. De fato, em cada intervalo $J_{j}^{p}$ a curva $\mathbf{x}_{\mathbf{r}}$ é suave por definição. Se $t \notin J_{j}^{p}$ para quaisquer $j=1, \ldots, m$ e $p \in \mathbb{Z}$ então pela proposição anterior

$$
t \notin \operatorname{supp} \xi_{j} \text { para todo } j=1, \ldots, m
$$

e daí existe $\epsilon>0$ tal que

$$
|s-t|<\epsilon \Rightarrow s \notin \operatorname{supp} \xi_{j} \text { para todo } j=1, \ldots, m \text {. }
$$

Desse modo, se $|s-t|<\epsilon$ temos

$$
\xi_{j}(s)=0 \text { para todo } j=1, \ldots, m
$$


e, portanto,

$$
\mathbf{e}^{\xi_{j}(s) X_{j}}\left(x_{\infty}\right)=x_{\infty} \text { para todo } j=1, \ldots, m
$$

Concluímos que se $|s-t|<\epsilon$ então

$$
\mathbf{x}_{\mathbf{r}}(s)=x_{\infty}
$$

Em particular, $\mathbf{x}_{\mathbf{r}}$ é suave em $(t-\epsilon, t+\epsilon)$. Isto prova que $\mathbf{x}_{\mathbf{r}}$ é suave em $\mathbb{R}$.

Da definição de $\mathbf{x}_{\mathbf{r}}$ temos, para $t \in I_{j}^{p}$, que

$$
\begin{aligned}
\mathbf{x}_{\mathbf{r}}{ }^{\prime}(t) & =\frac{d}{d t} \mathbf{e}^{\xi_{j}(t) X_{j}}\left(x_{\infty}\right) \\
& =\xi_{j}^{\prime}(t) X_{j}\left(\mathbf{e}^{\xi_{j}(t) X_{j}}\left(x_{\infty}\right)\right) \\
& =u_{j}^{r}(t) X_{j}\left(\mathbf{x}_{\mathbf{r}}(t)\right) \\
& =\sum_{k=1}^{m} u_{k}^{r}(t) X_{k}\left(\mathbf{x}_{\mathbf{r}}(t)\right)
\end{aligned}
$$

onde a última igualdade segue da Proposição A.2.

Agora, se $t \notin J_{j}^{p}$ para quaisquer $j=1, \ldots, m$ e $p \in \mathbb{Z}$ então de (A.2) segue que

$$
\mathbf{x}_{\mathbf{r}}^{\prime}(t)=0
$$

Nesse caso, contudo, a Proposição A.1 nos diz que $t \notin \operatorname{supp} u_{j}^{r}$ para todo $j=1, \ldots, m$, de modo que também podemos escrever

$$
\mathbf{x}_{\mathbf{r}}^{\prime}(t)=\sum_{k=1}^{m} u_{k}^{r}(t) X_{k}\left(\mathbf{x}_{\mathbf{r}}(t)\right)
$$

Os argumentos anteriores provam que $\left(\mathbf{x}_{\mathbf{r}}, u_{1}^{r}, \ldots, u_{m}^{r}\right)$ é uma trajetória do sistema (3.1). Ainda, a periodicidade de $\xi_{j}$ para todo $j=1, \ldots, m$ implica que $\mathbf{x}_{\mathbf{r}}$ definida acima é uma curva $T$-periódica. Ou seja, provamos que $\left(\mathbf{x}_{\mathbf{r}}, u_{1}^{r}, \ldots, u_{m}^{r}\right)$ conforme definida acima é uma trajetória de referência $T$ periódica para o sistema (3.1) nos moldes da Definição 3.1.

\section{A.2 Famílias regulares}

Definição A.5. Dizemos que $\Gamma$ é uma família regular se

$$
\operatorname{span}\left\{\operatorname{ad}\left(X_{1}\right)^{n} X_{k} ; 1 \leq k \leq m, n \in \mathbb{N}\right\}=\operatorname{Lie}(\Gamma)
$$

Provaremos agora que se $\Gamma$ é uma família regular então a trajetória $\left(\mathbf{x}_{\mathbf{r}}, u_{1}^{r}, \ldots, u_{m}^{r}\right)$ construída 
na seção anterior é regular i.e.

$$
\operatorname{span}\left\{\operatorname{Ad}\left(\mathbf{x}_{\mathbf{r}}\right) X_{k} ; 1 \leq k \leq m\right\}=\operatorname{Lie}(\Gamma) .
$$

De fato, denotemos

$$
\mathcal{V} \doteq \operatorname{span}\left\{\operatorname{Ad}\left(\mathbf{x}_{\mathbf{r}}\right) X_{k} ; 1 \leq k \leq m\right\}
$$

o qual necessariamente é um subespaço vetorial de Lie( $\Gamma)$ : recordemos que segundo o Corolário 2.13 temos

$$
\operatorname{Lie}(\Gamma)=\operatorname{span}\left\{\operatorname{Ad}(x) X ; x \in \mathcal{O}_{\Gamma}, X \in \Gamma\right\}
$$

e por definição de trajetória temos que $\mathbf{x}_{\mathbf{r}}(t) \in \mathcal{O}_{\Gamma}$ para todo $t \in \mathbb{R}$.

Para cada $k=1, \ldots, m$ defina $\Lambda_{k}^{0} \doteq X_{k}$ e $\lambda_{k}: \mathbb{R} \rightarrow \mathfrak{g}$ por

$$
\lambda_{k} \doteq \operatorname{Ad}\left(\mathbf{x}_{\mathbf{r}}\right) \Lambda_{k}^{0}
$$

Observe que por definição $\lambda_{k}(t) \in \mathcal{V}$ para todo $t \in \mathbb{R}$, de modo que todas as suas derivadas desfrutam desta mesma propriedade, ou seja

$$
\lambda_{k}^{(n)}(t) \in \mathcal{V} \text { para todo } t \in \mathbb{R}
$$

para todo $n \in \mathbb{N}$. Recordemos que o Lema 4.10 nos diz que

$$
\begin{aligned}
\lambda_{k}^{(n)} & =\operatorname{Ad}\left(\mathbf{x}_{\mathbf{r}}\right) \Lambda_{k}^{n} \\
\Lambda_{k}^{n+1} & \doteq\left(\Lambda_{k}^{n}\right)^{\prime}+\operatorname{ad}\left(X_{r}\right) \Lambda_{k}^{n}
\end{aligned}
$$

para todo $n \in \mathbb{N}$ onde

$$
X_{r}=\sum_{j=1}^{m} u_{j}^{r} X_{j}
$$

O lema a seguir não depende da construção de $\left(\mathbf{x}_{\mathbf{r}}, u_{1}^{r}, \ldots, u_{m}^{r}\right)$.

Lema A.6. Para cada $n \in \mathbb{N}$ podemos escrever

$$
\Lambda_{k}^{n}=\Delta_{n} X_{k}+a d\left(X_{r}\right)^{n} X_{k}
$$

onde

- $\Delta_{0}=\Delta_{1}=0 e$

- para $n \geq 2, \Delta_{n}$ é uma soma de produtos com a seguinte propriedade: em cada uma de suas 
parcelas aparece como fator pelo menos uma derivada de alguma ordem de ad $\left(X_{r}\right)$.

Demonstração. Primeiramente calculamos, utilizando a recorrência dada pelo Lema 4.10,

$$
\begin{aligned}
\Lambda_{k}^{0} & =X_{k} \\
\Lambda_{k}^{1} & =\operatorname{ad}\left(X_{r}\right) X_{k} \\
\Lambda_{k}^{2} & =\operatorname{ad}\left(X_{r}^{\prime}\right) X_{k}+\operatorname{ad}\left(X_{r}\right)^{2} X_{k}
\end{aligned}
$$

de modo que

$$
\begin{aligned}
& \Delta_{0}=0 \\
& \Delta_{1}=0 \\
& \Delta_{2}=\operatorname{ad}\left(X_{r}^{\prime}\right)
\end{aligned}
$$

o que prova nossa tese nos casos $n=0,1,2$ e utilizamos como base de indução. Supondo agora que a tese vale para um dado $n \geq 2$ temos

$$
\begin{aligned}
\Lambda_{k}^{n+1} & =\left(\Lambda_{k}^{n}\right)^{\prime}+\operatorname{ad}\left(X_{r}\right) \Lambda_{k}^{n} \\
& =\left(\Delta_{n} X_{k}+\operatorname{ad}\left(X_{r}\right)^{n} X_{k}\right)^{\prime}+\operatorname{ad}\left(X_{r}\right)\left\{\Delta_{n} X_{k}+\operatorname{ad}\left(X_{r}\right)^{n} X_{k}\right\} \\
& =\left(\Delta_{n}\right)^{\prime} X_{k}+\left(\operatorname{ad}\left(X_{r}\right)^{n}\right)^{\prime} X_{k}+\operatorname{ad}\left(X_{r}\right) \Delta_{n} X_{k}+\operatorname{ad}\left(X_{r}\right)^{n+1} X_{k} \\
& =\Delta_{n+1} X_{k}+\operatorname{ad}\left(X_{r}\right)^{n+1} X_{k}
\end{aligned}
$$

onde

$$
\Delta_{n+1} \doteq\left(\Delta_{n}\right)^{\prime}+\left(\operatorname{ad}\left(X_{r}\right)^{n}\right)^{\prime}+\operatorname{ad}\left(X_{r}\right) \Delta_{n}
$$

Como $\Delta_{n}$ por hipótese é uma soma de produtos tal que em cada uma de suas parcelas aparece como fator pelo menos uma derivada de alguma ordem $\operatorname{ad}\left(X_{r}\right)$ então é claro que $\left(\Delta_{n}\right)^{\prime}$ e $\operatorname{ad}\left(X_{r}\right) \Delta_{n}$ também necessariamente desfrutam desta propriedade. Ademais, uma conta simples mostra que

$$
\left(\operatorname{ad}\left(X_{r}\right)^{n}\right)^{\prime}=\sum_{p=1}^{n} \operatorname{ad}\left(X_{r}\right)^{p-1} \cdot \operatorname{ad}\left(X_{r}^{\prime}\right) \cdot \operatorname{ad}\left(X_{r}\right)^{n-p}
$$

de modo que esta parcela também desfruta da propriedade em questão. Logo $\Delta_{n+1}$ é uma soma de parcelas que desfrutam da supracitada propriedade, e portanto $\Delta_{n+1}$ também o faz. Isto verifica o passo de indução e conclui a demonstração.

No caso em que $\left(\mathbf{x}_{\mathbf{r}}, u_{1}^{r}, \ldots, u_{m}^{r}\right)$ denota a trajetória construída na seção anterior temos o seguinte: se $t_{1} \in I_{1}^{0}$ for fixado a Proposição A.3 nos diz que

- $u_{1}^{r}\left(t_{1}\right)=1$,

- $u_{j}^{r}\left(t_{1}\right)=0$ se $j \neq 1$ e 
- todas as derivadas de $u_{j}^{r}$ se anulam em $t_{1}$ para $j=1, \ldots, m$.

Daí temos que

$$
X_{r}\left(t_{1}\right)=\sum_{j=1}^{m} u_{j}^{r}\left(t_{1}\right) X_{j}=X_{1}
$$

e

$$
X_{r}^{(n)}\left(t_{1}\right)=0 \text { para todo } n \geq 1
$$

Esta observação implica, de acordo com o lema anterior, que $\Delta_{n}\left(t_{1}\right)=0$ para todo $n \in \mathbb{N}$ de modo que

$$
\Lambda_{k}^{n}\left(t_{1}\right)=\Delta_{n}\left(t_{1}\right) X_{k}+\operatorname{ad}\left(X_{r}\left(t_{1}\right)\right)^{n} X_{k}=\operatorname{ad}\left(X_{1}\right)^{n} X_{k}
$$

para todo $n \in \mathbb{N}$. Assim, da relação (A.3) no ponto $t=t_{1}$ temos que

$$
\lambda_{k}^{(n)}\left(t_{1}\right)=\operatorname{Ad}\left(\mathbf{x}_{\mathbf{r}}\left(t_{1}\right)\right) \Lambda_{k}^{n}\left(t_{1}\right)=\operatorname{Ad}\left(\mathbf{x}_{\mathbf{r}}\left(t_{1}\right)\right) \operatorname{ad}\left(X_{1}\right)^{n} X_{k}
$$

é um elemento de $\mathcal{V}$ para quaisquer $k=1, \ldots, m$ e $n \in \mathbb{N}$.

Desse modo, se denotarmos

$$
\mathcal{V}_{1} \doteq \operatorname{span}\left\{\operatorname{ad}\left(X_{1}\right)^{n} X_{k} ; 1 \leq k \leq m, n \in \mathbb{N}\right\}
$$

temos da relação anterior que

$$
\operatorname{Ad}\left(\mathbf{x}_{\mathbf{r}}\left(t_{1}\right)\right)\left(\mathcal{V}_{1}\right) \subset \mathcal{V}
$$

Finalmente, se supusermos que $\Gamma$ é família regular i.e. $\mathcal{V}_{1}=\operatorname{Lie}(\Gamma)$ temos, sendo $\operatorname{Ad}\left(\mathbf{x}_{\mathbf{r}}\left(t_{1}\right)\right)$ inversível, que

$$
\mathcal{V}=\operatorname{Lie}(\Gamma)
$$

ou seja $\left(\mathbf{x}_{\mathbf{r}}, u_{1}^{r}, \ldots, u_{m}^{r}\right)$ é uma trajetória regular. Em particular provamos o seguinte

Teorema A.7. Se $\Gamma$ é uma família regular então o sistema (3.1) possui trajetórias de referência $T$-periódicas regulares, para qualquer $T>0$ e satisfazendo condições iniciais arbitrárias em $\mathcal{O}_{\Gamma}$.

\section{A.3 Comentários e referências}

A construção da trajetória de referência apresentada na Seção A.1 é uma adaptação daquela introduzida em [Sil09], páginas 94 e 95. Nossa construção, apesar de um pouco menos concreta, tem a vantagem de produzir uma trajetória (curva integral mais controles) suave, em contraste 
com aquela de [Sil09] que é apenas suave por partes. Ademais, nossa construção não supõe que $\Gamma$ é Lie-determinada, outra melhoria.

Nosso conceito de família regular é análogo ao de p-controlabilidade introduzido por [Sil09], Definição 4.12, página 94, porém mais forte: nossa regularidade sempre implica 1-controlabilidade, mas não sabemos em que condições alguma p-controlabilidade implica regularidade. Esse ingrediente é um mero coadjuvante, agindo como condição suficiente - mas certamente não necessária - para obtermos uma trajetória regular, esta sim uma propriedade fundamental no desenvolvimento do Capítulo 5 do presente trabalho. Este nosso conceito de família regular também não possui ligação direta com o de sistema T-regular introduzido por [Sil09], Definição 4.2, página 85. Curiosamente porém as equações obtidas acima para $\lambda_{k}^{(n)}$ e $\Lambda_{k}^{n}$ são notavelmente parecidas com aquelas introduzidas por [Sil09] para definir T-regularidade (veja também nosso Lema 4.10), uma coincidência que até o momento não compreendemos plenamente. 


\section{Lista de Notações}

\begin{tabular}{|c|c|}
\hline $\operatorname{GL}(\mathcal{V})$ & grupo de Lie dos operadores lineares inversíveis $\mathcal{V} \rightarrow \mathcal{V}$ \\
\hline $\mathfrak{g l}(\mathcal{V})$ & álgebra de Lie dos operadores lineares $\mathcal{V} \rightarrow \mathcal{V}$ \\
\hline $\operatorname{tr}$ & traço \\
\hline $\operatorname{span} C$ & menor subespaço vetorial que contém $C \subset \mathcal{V}$ \\
\hline$C_{c}^{\infty}(I)$ & funções suaves $\mathbb{R} \rightarrow \mathbb{R}$ com suporte compacto contido em $I$ \\
\hline supp $f$ & suporte da função $f$ \\
\hline$\Re z, \Im z$ & parte real e parte imaginária de $z \in \mathbb{C}$ \\
\hline$C^{\infty}(M)$ & funções suaves $M \rightarrow \mathbb{R}$ \\
\hline$T_{x} M$ & espaço tangente em $x \in M$ \\
\hline$M$ & fibrado tangente de $M$ \\
\hline$d \phi, d \phi_{x}$ & diferencial de $\phi($ no ponto $x)$ \\
\hline Diff $M$ & difeomorfismos $M \rightarrow M$ \\
\hline $\operatorname{Vec} M$ & campos vetoriais suaves em $M$ \\
\hline & pushforward por um difeomorfismo $\phi$ \\
\hline$\left\{\mathbf{e}^{t X}\right\}_{t \in \mathbb{R}}$ & subgrupo a um parâmetro gerado por $X \in \operatorname{Vec} M$ \\
\hline $\operatorname{Lie}(\Gamma)$ & menor subálgebra de Lie que contém $\Gamma \subset$ Vec $M$ \\
\hline $\operatorname{Gr}(\Gamma)$ & subgrupo gerado por $\left\{e^{t X} ; X \in \Gamma, t \in \mathbb{R}\right\}$ \\
\hline $\mathcal{O}_{\Gamma}(x)$ & órbita de $\Gamma$ que contém $x \in M$ \\
\hline ad & representação adjunta de uma álgebra de Lie \\
\hline$B$ & forma de Killing \\
\hline $\mathfrak{z}(\mathfrak{g})$ & centro de $\mathfrak{g}$ \\
\hline$L_{x}, R_{x}$ & translações à esquerda e à direita por $x \in G$ \\
\hline $\mathbf{e}^{X}$ & aplicação exponencial \\
\hline$I_{x}$ & conjugação por $x \in G$ \\
\hline Ad & representação adjunta de um grupo de Lie \\
\hline$Z(G)$ & centro de $G$ \\
\hline $\mathcal{O}_{\Gamma}$ & órbita de uma família invariante à esquerda $\Gamma$ \\
\hline$V$ & função "tipo Lyapunov" (caractere de Ad) \\
\hline$W$ & campo auxiliar \\
\hline$E_{V}$ & pontos de equilíbrio de $W$ \\
\hline$\left.x_{0}\right)$ & conjunto $\Omega$-limite \\
\hline
\end{tabular}




\section{Referências Bibliográficas}

[AS04] A. A. Agrachev and Yu. L. Sachkov. Control Theory from the Geometric Viewpoint, volume 87 of Encyclopaedia of Mathematical Sciences. Springer, 2004.

[BL05] F. Bullo and A. D. Lewis. Geometric Control of Mechanical Systems, volume 49 of Texts in Applied Mathematics. Springer, 2005.

[Jur97] V. Jurdjevic. Geometric Control Theory, volume 51 of Cambridge Studies in Advanced Mathematics. Cambridge University Press, 1997.

[Kha02] H. K. Khalil. Nonlinear Systems. Prentice Hall, third edition, 2002.

[Kna02] A. W. Knapp. Lie Groups Beyond an Introduction, volume 140 of Progress in Mathematics. Birkhäuser, second edition, 2002.

[Lee03] J. M. Lee. Introduction to Smooth Manifolds, volume 218 of Graduate Texts in Mathematics. Springer, 2003.

[Mil63] J. W. Milnor. Morse Theory, volume 51 of Annals of Mathematics Studies. Princeton University Press, 1963.

[Sac09] Yu. L. Sachkov. Control theory on lie groups. Journal of Mathematical Sciences, 156(3):381-439, 2009.

[SdSR09] H. B. Silveira, P. S. Pereira da Silva, and P. Rouchon. A time-periodic lyapunov approach for motion planning of controllable driftless systems on $\mathrm{SU}(n)$. Proc. of the 48th IEEE Conf. on Decision and Control - CDC, pages 363-368, 2009.

[Sep07] M. R. Sepanski. Compact Lie Groups, volume 235 of Graduate Texts in Mathematics. Springer, 2007.

[Sil09] H. B. Silveira. Formas Triangulares para Sistemas Não-Lineares com Duas Entradas e Controle de Sistemas Sem Arrasto em SU(n) com Aplicações em Mecânica Quântica. Tese de Doutorado pela Escola Politécnica da Universidade de São Paulo - EPUSP. 2009 .

[Sot79] J. Sotomayor. Lições de Equações Diferenciais Ordinárias. Projeto Euclides. Instituto de Matemática Pura e Aplicada (IMPA), 1979.

[Vid02] M. Vidyasagar. Nonlinear Systems Analysis, volume 42 of Classics in Applied Mathematics. Society for Industrial and Applied Mathematics (SIAM), second edition, 2002. 


\section{Índice Remissivo}

$B, 12$

$C^{\infty}(M), 1$

$E_{W}, 29$

$I_{x}, 14$

$L_{x}, 13$

$R_{x}, 13$

$T M, 1$

$T_{x} M, 1$

$V, 29$

$W, 29$

$Z(G), 22$

Ad, 14

$\operatorname{Ad}_{\mathbb{C}}, 62$

$\Omega_{W}, 51$

$\Omega_{X}\left(t_{0}, x_{0}\right), 35$

ad, 11

Diff $M, 2$

$\mathbf{e}^{t X}$

aplicação exponencial, 14

elemento de Diff $M, 2$

elemento de $G, 14$

$\mathfrak{g}_{\mathbb{C}}, 62$

$\operatorname{Gr}(\Gamma), 4$

$\operatorname{Lie}(\Gamma), 4$

$\operatorname{Lie}_{x}(\Gamma), 4$

$\mathfrak{z}(\mathfrak{g}), 12$

$\mathcal{O}_{\Gamma}, 18$

$\mathcal{O}_{\Gamma}(x), 4$

$\phi_{*}, 2$

Vec $M, 2$

$a_{k}, 29$

$d \phi, 1$

e, 13

álgebra de Lie

de um grupo de Lie, 13

redutiva, 12

semi-simples, 12

órbita, 4 de uma família invariante à esquerda, 18

aplicação exponencial, veja $\mathbf{e}^{t X}$

atrator local, 29

campo auxiliar, 29

campo vetorial, 2

completo, 2

invariante à esquerda, 13

centro

de um grupo de Lie $G, 22$

de uma álgebra de Lie $\mathfrak{g}, 12$

complexificação, 62

conjugação, 14

conjunto $\Omega$-limite, 35

controle, 25

coordenadas de segunda espécie, 60

diferencial, 1

erro de rastreamento, 26

família

invariante à esquerda, 18

Lie-determinada, 8

regular, 74

forma de Killing, 12

não-degenerada, 12

função

"tipo Lyapunov", 29

não decrescente ao longo de um campo, 35

periódica, 39

grupo de Lie semi-simples, 23

ideal, 12

identidade de Jacobi, 11

Lema de Barbalat, 47

corolário, 48 
matriz Hessiana, 60

ponto $\Omega$-limite, 35

ponto crítico não-degenerado, 59

problema do planejamento periódico, 26

produto interno Ad-invariante, 21

pushforward, 2

representação adjunta

de um grupo de Lie, 15

de uma álgebra de Lie, 11

subgrupo a um parâmetro, 2

Teorema

da Órbita, 4

da Órbita para grupos de Lie, 19

de Rashevsky-Chow, 8

trajetória, 25

de referência periódica, 26

regular, 55

translação, 13

variedade, 1 